\title{
DEVELOPMENT OF NOVEL SITE-SPECIFIC IMAGING PROBES FOR PROSTATE CANCER DETECTION
}

\author{
By
}

Hang Xu

Submitted to the Doctoral Committee

The Department of Chemical Engineering

University of Missouri

Ph.D. defense

in partial fulfillment of the requirements for the $\mathrm{Ph}$. D. program

Dr. Lixin Ma, Dissertation Supervisor

December 2017 
The undersigned, appointed by the dean of the Graduate School, have examined the dissertation entitled

DEVELOPMENT OF NOVEL SITE-SPECIFIC IMAGING PROBES FOR PROSTATE CANCER DETECTION

presented by Hang Xu,

a candidate for the degree of doctor of philosophy, and hereby certify that, in their opinion, it is worthy of acceptance.

Professor Lixin Ma

Professor Baolin Deng

Professor Ping Yu

Professor Bret Ulery

Professor Yangchuan Xing 



\section{ACKNOWLEDGEMENT}

I cannot express enough thanks to my advisor, Prof. Lixin Ma, for her valuable guidance and encouragement to me. She has provided me extensive professional supervision and taught me a great deal of both scientific research and life in general. As an educator, she has shown me, by her example, what a good scientist, or person, should be. Without her initiation, advice, or expertise, the research and dissertation wouldn't have happened.

I'm very grateful for Prof. Baolin Deng, Prof. Yangchuan Xing, Prof. Bret Ulery, and Prof. Ping Yu for their insightful comments and encouragement. I have a genuine appreciation for the learning opportunity offered by my committee. I would like to especially thank Prof. Ping Yu, for the insight, instructions, and collaborations from him and his research group on my research projects throughout my Ph. D. study.

I would like to thank my collaborators, Dr. Rajendra Bandari, for his help in IC50 measurements, Dr. Zongrun Jiang, for his instruction in HPLC practice, Li Lee, for her assistance in fluorescence determination and animal work, Fengfei Wang and Dr. Miao Zhang, for their instructions in FTIR and DLS sample preparations and measurements, Dr. Ming Yang, for his instruction on MRI practice. My sincere thanks also go to Prof. Timothy Hoffman and Prof. Jeffrey Smith for their continued support in my research. 
Finally, I am grateful to my parents, Xu, Huageng and Hu, Xingzhen for their spiritual support to me throughout my research and dissertation writing. 


\section{Table of Contents}

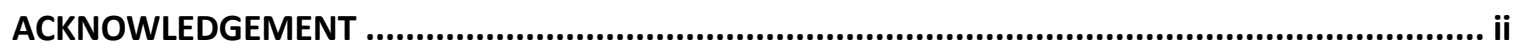

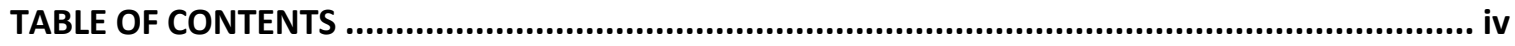

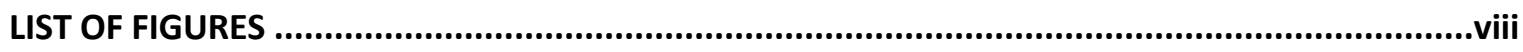

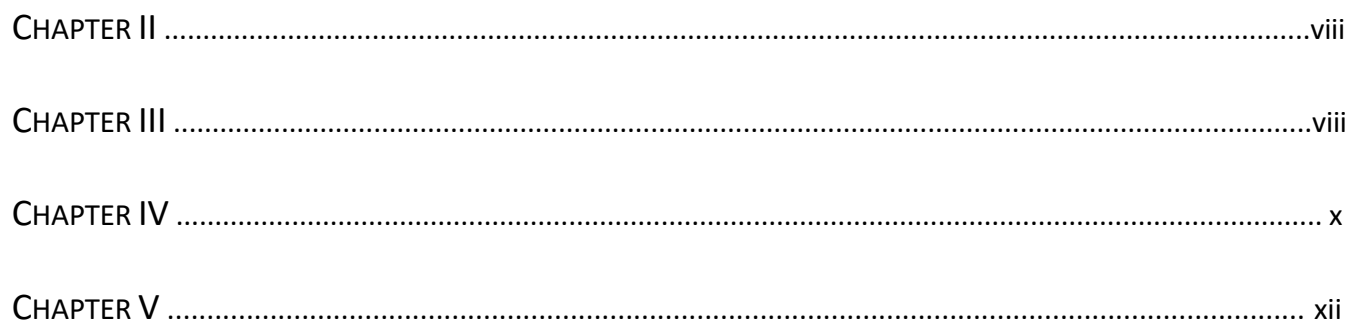

LIST OF TABLES

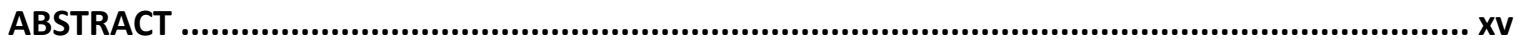

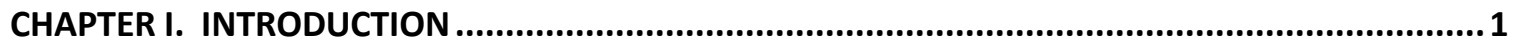

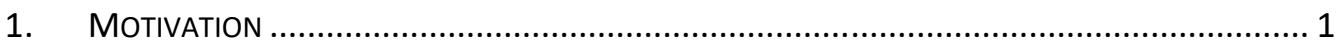

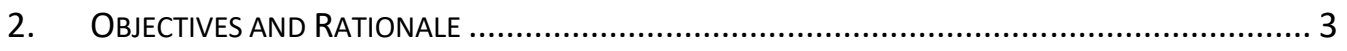

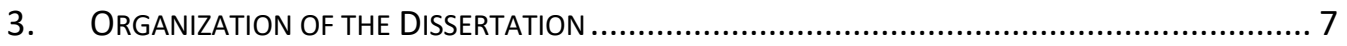

CHAPTER II. BACKGROUND

1. NANOPARTICLES BASED MOLECULAR IMAGING AND TARGETING STRATEGIES ......................... 8

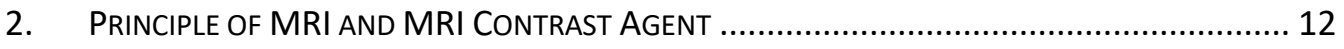

Physics behind Magnetic Resonance Imaging ................................................... 12

Magnetic Resonance Imaging Procedure ........................................................ 14 
3. The Principle of Photoacoustic Tomography And Photoacoustic Contrast Agent...

4. NIRF IMAGING AND IMAGING PROBES.

\section{CHAPTER III. BIMODAL MRI/FLUORESCENCE IMAGING CONTRAST AGENT TARGETING HUMAN}

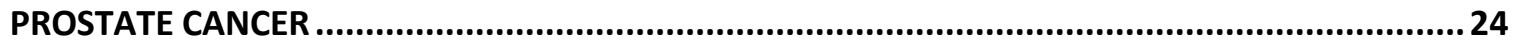

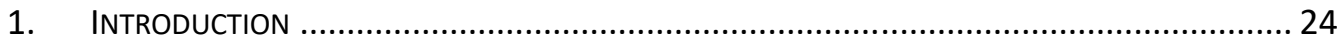

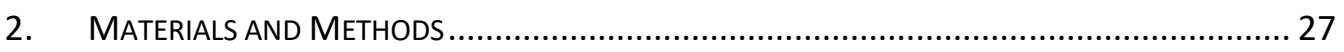

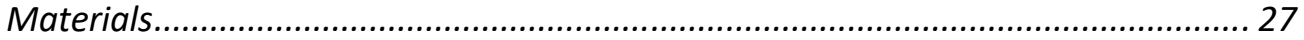

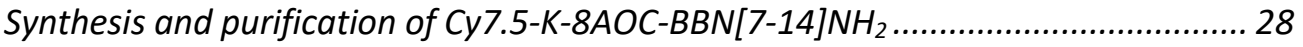

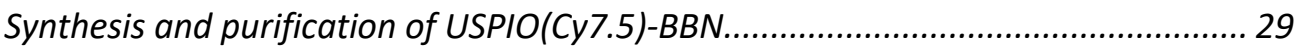

Determination of iron content of nanoparticles .................................................... 31

Determination of peptide to nanoparticle ratio......................................................... 32

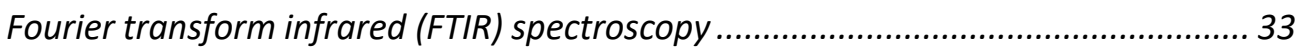

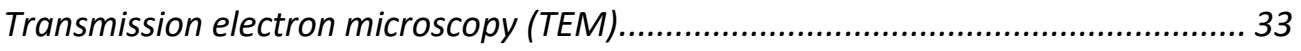

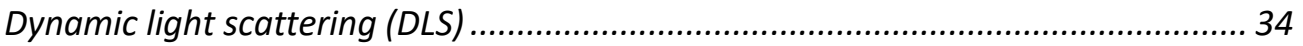

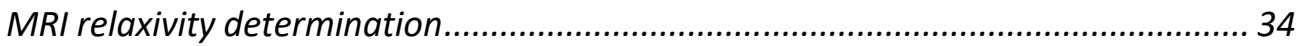

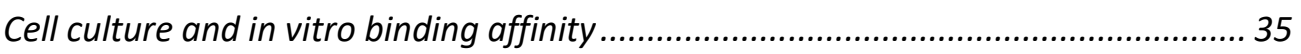

In vitro cellular microscopic imaging: uptake, blocking, internalization, Prussian

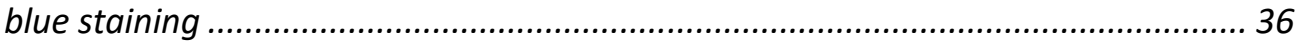

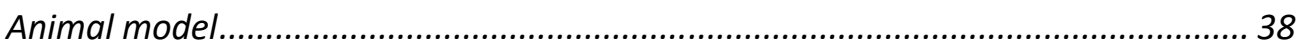

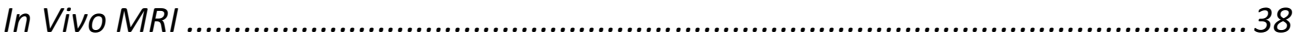

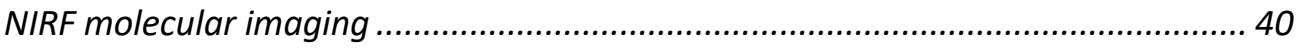

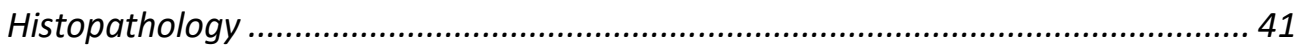

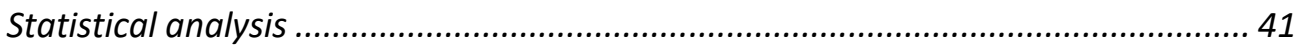




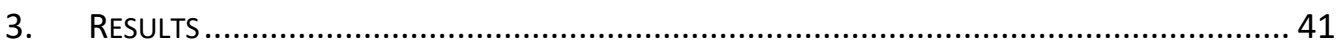

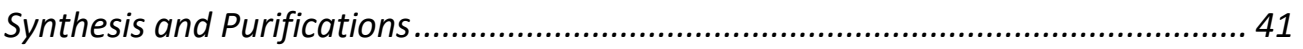

Core size and hydrodynamic size determination .................................................... 44

MRI Relaxivity Measurements of USPIO(Cy7.5)-BBN.............................................. 45

Binding Affinity of USPIO(Cy7.5)-BBN to Prostate Cancer Cells .................................46

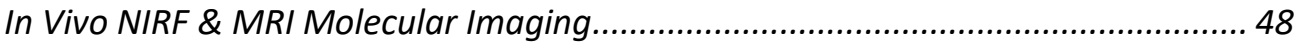

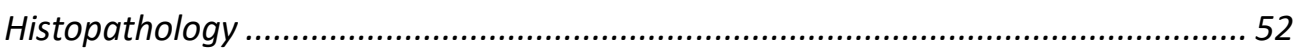

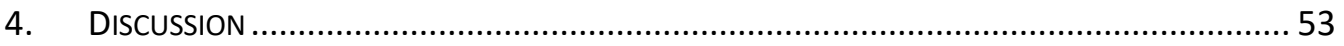

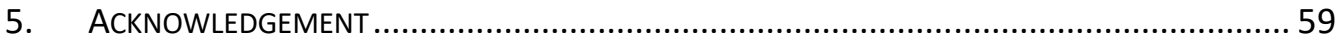

\section{CHAPTER IV. CANCER CELL SPECIFIC SILICA-COATED IRON OXIDE NANOPARTICLES FOR}

PHOTOACOUSTIC DETECTION OF PROSTATE CANCER ............................................................60

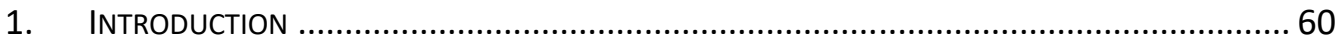

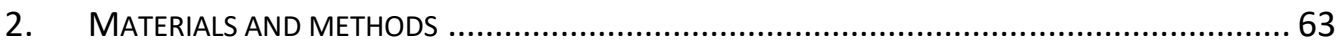

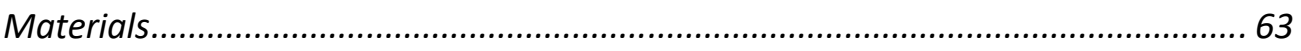

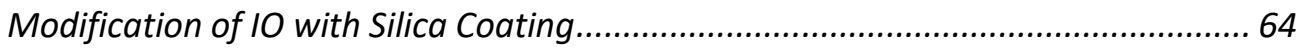

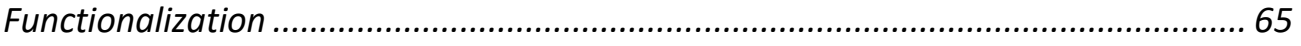

Conjugation of AF750-K-8Aoc-BBN[7-14]NH2 (AF750-BBN) ............................... 65

Conjugation of AF750-K-8Aoc-BBN[7-14]NH2- with COOH Functionalized Silica

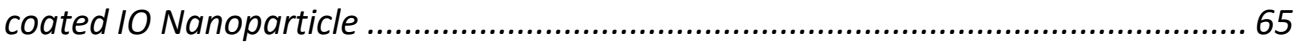

FTIR

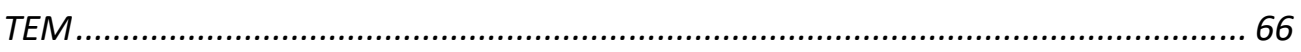

Determination of iron content of nanoparticles ......................................................6. 67

Determination of peptide to nanoparticle ratio.................................................... 68

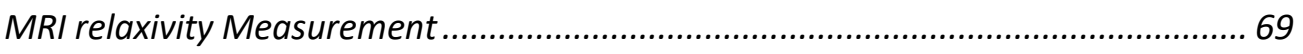




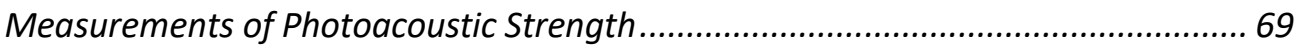

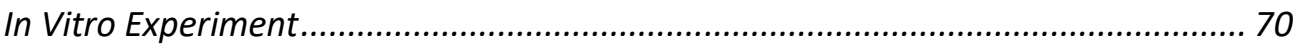

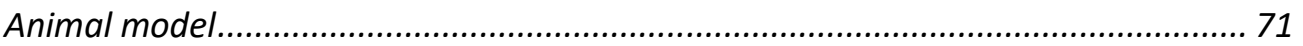

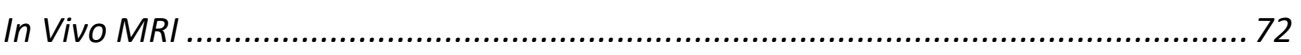

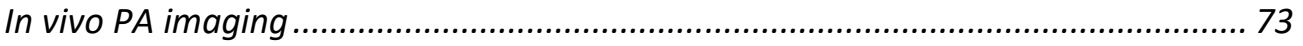

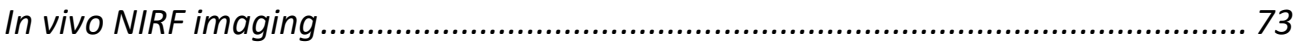

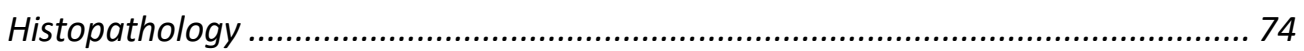

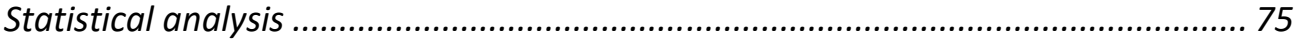

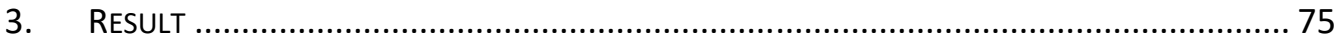

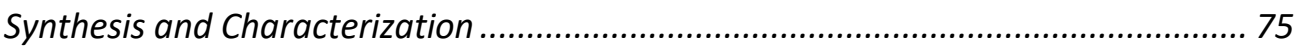

In vivo PA imaging of SIO-AF750-BBN in PC-3 tumor mouse model .........................8 85

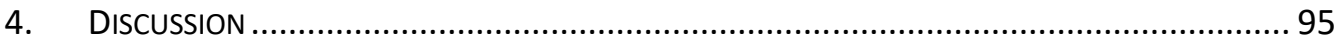

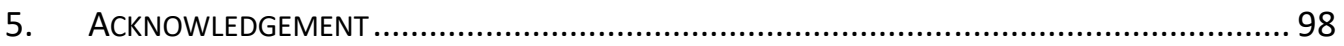

\section{CHAPTER V. NEAR-INFRARED FLUORESCENCE DYE LABELED BOMBESIN ANTAGONIST}

\section{PEPTIDES FOR MOLECULAR IMAGING OF GRPR: AN IN VITRO AND IN VIVO EVALUATION IN}

HUMAN PROSTATE CANCER ............................................................................................99

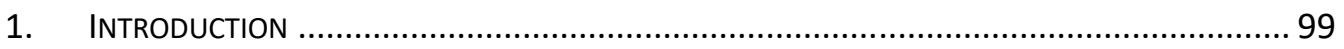

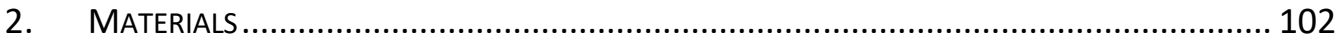

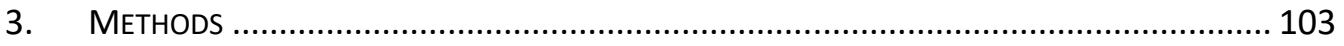

Chemical Conjugation, Purification and Mass Spectrum Examination ................... 103

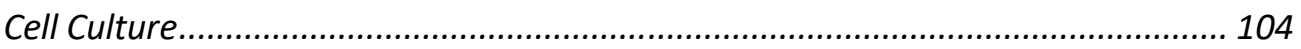

Cellular Uptake, Blocking and Internalization Assay ............................................ 104

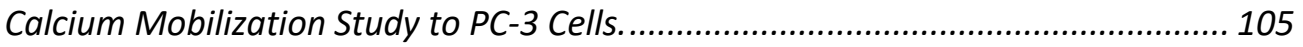

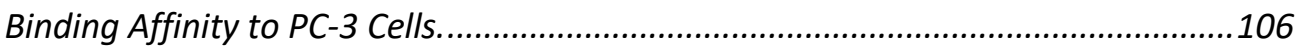




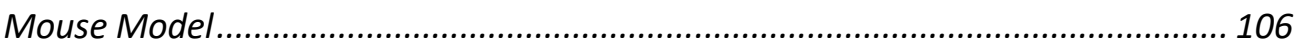

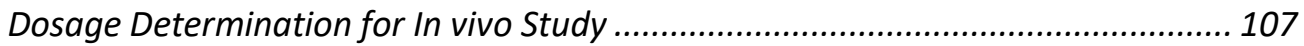

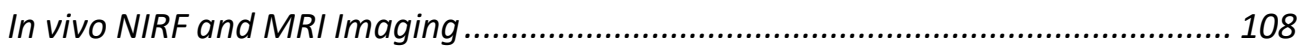

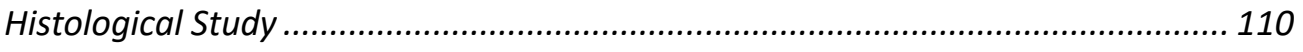

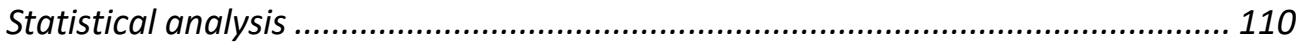

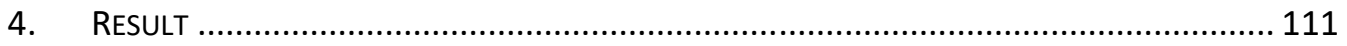

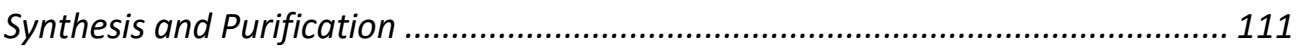

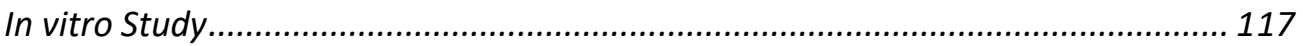

In vivo and ex Vivo NIRF Imaging Study........................................................... 121

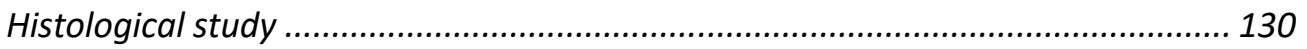

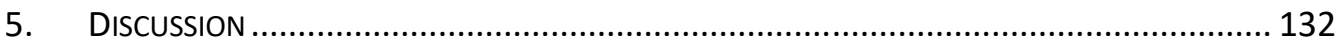

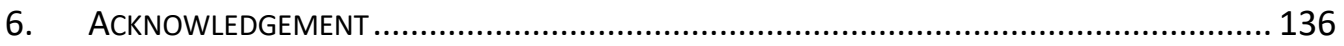

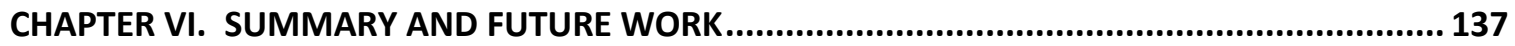

REFERENCE

VITA 


\section{List of Figures}

\section{Chapter II}

Figure 2.1 Applications of nanotechnologies in the field of medical imaging. ..................9

Figure 2.2 Influence on net magnetization by RF pulse ............................................... 14

Figure 2.3 Superparamagnetism of iron oxide nanoparticle ...................................... 17

\section{Chapter III}

Figure 3.1 Schematic diagram of interaction between USPIO(Cy7.5)-BBN and prostate cancer cells 26

Figure 3.2 Schematic diagram of the synthetic process for the USPIO(Cy7.5)-BBN

Figure 3.3 FTIR spectra of glucose coated USPIO nanoparticles (blue), pure casein (red) and casein coated USPIO nanoparticles (black).

Figure 3.4 HPLC profile of the BBN-Cy7.5 with a purity over $95 \%$.

Figure 3.5 Mass spectrum of the BBN-Cy7.5 or K(Cy7.5)-8AOC-BBN[7-14]NH2. The MW in the figure is $1840.0 \mathrm{Da}$, matching the theoretic $\mathrm{MW}, 1840.1 \mathrm{Da}$.

Figure 3.6 TEM of (A) glucose treated USPIO (step 1), (B) casein coated USPIO (step 2), (C) USPIO(Cy7.5)-BBN (step 3). (D) Histogram of the core diameter of USPIO(Cy7.5)-BBN.

Figure 3.7 Number weighted hydrodynamic radius of USPIO(Cy7.5)-BBN 45

Figure 3.8(A) T2-weighted MRI image at TR of 2500ms and TE of $55 \mathrm{~ms}$ for solutions with different concentrations of USPIO(Cy7.5)-BBN. (B) Linear fitting of relaxation rate at different concentration

Figure 3.9 Microscopic cellular images. (A) Uptake: PC-3 cells treated with USPIO(Cy7.5)BBN. (B) Blocking: PC-3 cells pre-treated with 400-fold BBN[1-14], followed by the 
incubation with USPIO(Cy7.5)-BBN. (C) Internalization: PC-3 cells treated with USPIO(Cy7.5)-BBN, and further washed with $\mathrm{pH}=2.5$ buffer. (D) Prussian blue staining test of USPIO(Cy7.5)-BBN (dark blue) bound to PC-3 cells in a bright field image. (E) Prussian blue staining test of USPIO(Cy7.5)-BBN (dark blue) bound to PC-3 cells in a fluorescence image (red represents Cy7.5).

Figure 3.10 In vitro binding affinity of USPIO(Cy7.5)-BBN in PC-3 cells. IC50 $=2.5 \pm 0.7 \mathrm{nM}$, by $125 \mathrm{I}-\mathrm{Ty}$ r4-BBN competitive binding assay. 48

Figure 3.11 IVIS imaging of tail vein injection of USPIO(Cy7.5)-BBN in SCID mice bearing PC-3 tumors for pre-injection(A), the uptake group at $48 \mathrm{hr}$ post injections(B) and the blocking group at $48 \mathrm{hr}$ post injection(C). The left is the ex vivo NIRF imaging of organs collected $48 \mathrm{~h}$ post injection for the uptake group(D) and the blocking group(E). Number denotes: $\mathrm{H}$, heart; Lu, lung; Li, liver; Sp, spleen; Ki, kidney; $\mathrm{Pa}$, pancreas; $\mathrm{Bl}$, bladder; $\mathrm{Tu}$, tumor; Mu, muscle; Ti, tibia. 50

Figure 3.12(A) Representative T2-weighted MRI of uptake group (upper panel) compared to the blocking group (lower panel) pre and post i.v. injections of USPIO(Cy7.5)-BBN. TR $=2347 \mathrm{~ms}$ and $\mathrm{TE}=33 \mathrm{~ms}$. (B)The contrast enhancement ratio (CER) of uptake and blocking groups at $4 \mathrm{hr}, 24 \mathrm{hr}$ and $48 \mathrm{hr}$ p.i. 51

Figure 3.13 Histopathological results: (A) Kidney in the uptake group. (B) Liver in the uptake group. (C, D) Tumors in the uptake group. (E) Kidney in the blocking group. (F) Tumor in the blocking group. Arrows indicate the USPIO nanoparticles with Prussian blue staining. The scale bar represents $100 \mathrm{um}$ 53

\section{Chapter IV}

Figure 4.1 Schematic diagram of silica coating, functionalization, and conjugations to obtain SIO-AF750-BBN 76

Figure 4.2 Molecular structures of AF750-K-8Aoc-BBN[7-14]NH2 (AF750-BBN). Only one of the two $\mathrm{NH} 2$ on lysine is conjugated with AF750. 77

Figure 4.3(A) HPLC profile (retention time: $17.7 \mathrm{~min}$ ) and (B) mass spectrum of AF750-K8Aoc-BBN[7-14] $\mathrm{NH}_{2}$ (AF750-BBN). Measured MW is $2075.9 \mathrm{Da}$, in good agreement with the theoretical MW of $2075.8 \mathrm{Da}$. 78

Figure 4.4 FTIR spectra of IO nanoparticle before and after silica coating 79

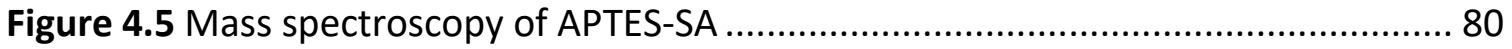

Figure 4.6 SIO-AF750-BBN and its NIR fluorescence imaging on an IVIS system 80 
Figure 4.7 TEM images of (A) silica coated iron oxide nanoparticles and (B) $\mathrm{COOH}$ functionalized silica coated iron oxide nanoparticle (C) SIO-AF750-BBN (D) Histogram of the overall diameters of SIO-AF750-BBN

Figure 4.8 $\mathrm{MRI} r_{2}$ relaxivity measurements of SIO-AF750-BBN and Feridex ${ }^{\circledR}$. (A) T2weighted $\mathrm{MRI}$ at varies concentrations, and $r_{2}$ fittings of (B) SIO-AF750-BBN and (C) Feridex ${ }^{\circledR}$

Figure 4.9 PA intensity of SIO as compared with USPIO(Cy7.5)-BBN and Feridex as references. The chart on the right displays the relative PA intensity of different compounds after concentration normalization

Figure 4.10 In vitro binding specificity of SIO-AF750-BBN to the GRP receptors on PC-3 cells. (A) NIR fluorescence image and (B) merged image (NIRF and bright-field) of SIOAF750-BBN in the uptake study in PC-3 cells; (C) NIR fluorescence image and (D) merged image (NIRF and bright-field) of SIO-AF750-BBN in the blocking study with the BBN[1-14] as the blocking agent in PC-3 cells 85

Figure 4.11 In vivo photoacoustic images (color scale) overlaid with ultrasound images (gray scale) pre- (left) and 1-hour post- (right) tail vein injection of SIO-AF750-BBN, uptake as compared to blocking with BBN[1-14], and AF750-Gpip-Sta-BBN, a small molecular BBN antagonist. The PA images shown are at $750 \mathrm{~nm}$ wavelength. Images were acquired using a VEVO 2100/VEVO LAZR system and an LZ250 PA transducer

Figure 4.12 Photoacoustic intensity enhancements [PA_post - PA_pre] in PC-3 tumor tissues (A) and the tumor surface vasculatures (B) in the wavelength range of $680 \mathrm{~nm}$ to $970 \mathrm{~nm}$ acquired on VEVO LAZR system. The SIO-AF750-BBN PA enhancement is increased 3-fold than the blocking group in the tumor tissues and 1.5-fold in the tumor surface vasculatures. The SIO-AF750-BBN PA enhancement is also 5 times than the small molecular AF750-Gpip-Sta-BBN group in the tumor tissues and the surface vasculatures.

Figure 4.13 In vivo NIRF study of SIO-AF750-BBN in a prostate tumor mouse model. (A)The IVIS image of the mice from the uptake group and the blocking group at $1 \mathrm{hr}$ post tail vein injection. (B) In vivo tumor to muscle ratio versus different time points ( $n=3$ for each group). The TMR is significantly higher in the uptake group as compared to the blocking group at all the time points $(p<0.05)$. 89

Figure 4.14 In vivo MRI study of SIO-AF750-BBN in SCID mouse model bearing PC-3 prostate cancer. (A) Representative MRI images: T2-weighted (T2W) MRI and T2-map of an axial slice across the tumor in a mouse 2 hours post injection of SIO-AF750-BBN. (B) The T2 relaxation time of tumor and muscle pre- and post-injection. There is no enhancement in the uptake group and the blocking group. T: Tumor, M: Muscle, B: bladder, and P: prostate. 90 
Figure 4.15 Ex vivo NIRF study of SIO-AF750-BBN with a prostate tumor mouse model. (A) The IVIS image of the organs from the uptake group as compared to the blocking group, letters denote: T: Tumor, Br: Brain, Pa: Pancreas, St: Stomach, H: Heart, M: Muscle, Pr: Prostate, LI: Large intestine, SI: Small intestine, Sp Spleen, Li Liver, Lu: Lungs, Bl: Bladder, K: Kidneys. (B) Bio-distribution of SIO-AF750-BBN-AF750 in organs in the uptake groups as compared to the blocking group ( $n=3$ for each group).

Figure 4.16 Images of histological slices regarding the SIO-AF750-BBN (Prussian blue staining) in tumors, pancreas, prostate, and muscles in the uptake group as compared to the blocking group. 94

\section{Chapter V}

Figure 5.1 Scheme of synthesis of bombesin antagonist with NIRF dyes. (B) The fluorescence profile for AF750-G-Pip-Sta-BBN 112

Figure 5.2 Molecular structures of AF750-linker-Sta-BBN analogs. (A) AF750-G-Pip-StaBBN, (B) AF750-GSG-Sta-BBN, and (C) AF750-6Ahx-Sta-BBN

Figure 5.3 HPLC profiles of the purified AF750 labeled Sta-BBN antagonist analogs. HPLC gradient: 20\%-40\% B buffer in $15 \mathrm{~min}$, flow rate $1 \mathrm{ml} / \mathrm{min}, \mathrm{A}$ buffer: 0.1 TFA in $\mathrm{H} 2 \mathrm{O}, \mathrm{B}$ buffer: 0.1 TFA in acetonitrile (ACN).

Figure 5.4 Mass spectra of AF750-G-pip-Sta-BBN (theoretical MW: 2177), AF750-GSGSta-BBN (theoretical MW: 2181) and AF750-6Ahx-Sta-BBN (theoretical MW: 2093) ... 116

Figure 5.5 Fluorescence spectrum of AF750-G-Pip-Sta-BBN.

Figure 5.6 In vitro NIRF microscopic images overlaid on the bright-field images for PC-3 cells treated with AF750-G-Pip-Sta-BBN (top row), AF750-GSG-Sta-BBN (middle row), and AF750-6Ahx-Sta-BBN (bottom row) in the uptake study (left), the blocking study (middle) and the internalization study (right). The red represents AF750 fluorescence signals.

Figure 5.7 The fitting curves for calcium mobilization study for BBN[1-14] (BBN agonist) and AF750 labeled BBN antagonist

Figure 5.8 IC50 value of AF750-G-Pip-Sta-BBN/ and AF750-GSG-Sta-BBN against 125ITyr4-BBN in PC-3 cells is $9.8 \pm 2.9 \mathrm{nM}$ and $16.5 \pm 4.6 \mathrm{nM}$, respectively. 120

Figure 5.9 In vivo NIRF imaging (Ex: $745 \mathrm{~nm} / \mathrm{Em}: 800 \mathrm{~nm}$ ) of SCID mice bearing PC-3 tumor xenograft on the right flank at 1 hour post tail vein injection of AF750-G-Pip-Sta-BBN (left), AF750-GSG-Sta-BBN (middle) and AF750-6Ahx-Sta-BBN (right), respectively, for 
the uptake group (A, C, E) as compared to the blocking group (B, D, F). Corresponding T2-weighted MRI of the tumor cross section is shown below each NIRF image (G-L). Dotted line indicates the MRI cross section location 123

Figure 5.10 In vivo kinetic tumor NIRF signal strength(Ex: $745 \mathrm{~nm} / \mathrm{Em}: 800 \mathrm{~nm}$ ) of SCID mice bearing PC-3 tumor xenograft on the right flank for AF750-G-Pip-Sta-BBN (A)(N=2 for the uptake and $\mathrm{N}=1$ for the blocking), AF750-GSG-Sta-BBN (B)(N=1 for the uptake and $\mathrm{N}=1$ for the blocking) and AF750-6Ahx-Sta-BBN (C) ( $\mathrm{N}=3$ for the uptake and $\mathrm{N}=3$ for the blocking), respectively. 124

Figure 5.11 Ex vivo NIRF imaging (Ex: $745 \mathrm{~nm} / \mathrm{Em}: 800 \mathrm{~nm}$ ) of tumor, pancreas, muscle, and prostate in the uptake group as compared to the blocking group for AF750-G-PipSta-BBN (left), AF750-GSG-Sta-BBN (middle), and AF750-6Ahx-Sta-BBN (right). The color

bar represents the average radiance efficiency $(\mathrm{p} / \mathrm{sec} / \mathrm{cm} 2 / \mathrm{sr} \mu \mathrm{W} / \mathrm{cm} 2)$. 125

Figure 5.12 Ex vivo NIRF image of (A) uptake and (B) blocking representative animals, and (C) bio-distribution data for AF750-G-Pip-Sta-BBN at 2.5 hour p.i. ${ }^{*}$ indicates a significant difference by the student t-test, $\mathrm{p}<0.5$. Letters denote: $\mathrm{T}$ : Tumor, $\mathrm{Br}$ : Brain, Pa: Pancreas, St: Stomach, H: Heart, M: Muscle, Pr: Prostate, LI: Large intestine, SI: Small intestine, Sp: Spleen, Li: Liver, Lu: Lungs, Bl: Bladder, K: Kidneys. 127

Figure 5.13 Ex vivo NIRF image of (A) uptake and (B) blocking representative animals, and (C) bio-distribution data for AF750-GSG-Sta-BBN at 3.5 hours p.i. * indicates a significant difference by the student t-test, $\mathrm{p}<0.5$. Letters denote: $\mathrm{T}$ : Tumor, $\mathrm{Br}$ : Brain, Pa: Pancreas, St: Stomach, H: Heart, M: Muscle, Pr: Prostate, LI: Large intestine, SI: Small intestine, Sp: Spleen, Li: Liver, Lu: Lungs, Bl: Bladder, K: Kidneys 128

Figure 5.14 Ex vivo NIRF image of (A) uptake and (B) blocking representative animals, and (C) bio-distribution data for AF750-6Ahx-Sta-BBN at 2.5-hour p.i. * indicates a significant difference by the student $\mathrm{t}$-test, $\mathrm{p}<0.5$. Letters denote: $\mathrm{T}$ : Tumor, $\mathrm{Br}$ : Brain, Pa: Pancreas, St: Stomach, H: Heart, M: Muscle, Pr: Prostate, LI: Large intestine, SI: Small intestine, Sp: Spleen, Li: Liver, Lu: Lungs, BI: Bladder, K: Kidneys

Figure 5.15 Histological studies for the tumor, muscle, and prostate tissue slices in the uptake and blocking group for AF750-G-pip-Sta-BBN (first two rows), AF750-GSG-StaBBN (middle two rows) and AF750-6Ahx-Sta-BBN (last two rows). Red: AF750 dye; Blue: DAPI staining. 131 


\section{List of Tables}

Table 4.1 Characteristics of the synthesized antagonist NIRF-linker-Sta-BBN ................113 


\begin{abstract}
Molecular imaging is believed to play a pivotal role in decreasing cancer mortality and improving prognosis in the new era. It relies on molecular probes to image cellular or molecular abnormalities in living body. The primary objective of this research is to develop novel multimodal molecular imaging probes, via nanotechnology and peptide conjugation chemistry, for site-specific targeting and imaging of human prostate cancers.
\end{abstract}

A novel nanoparticle based bimodal MRI/fluorescence molecular imaging probe was developed, characterized, and studied for its capacity to target gastrin releasing peptide receptor (GRPr) which is overexpressed in human prostate cancer. Synthesized USPIO(Cy7.5)-BBN nanoparticles have a small core size $(5 \mathrm{~nm})$, a high MRI relaxivity, and a superior binding affinity and specificity to the GRPr overexpressed on human prostate cancer in vitro and in vivo. Furthermore, SIO-AF750-BBN, a silica-coated iron oxide nanoparticle loaded with near infrared fluorescence (NIRF) dyes and bombesin, was developed as a tri-modal photoacoustic (PA), NIRF and MRI imaging probe. SIO-AF750BBN displayed a high binding affinity and specificity to the GRPr expressed prostate cancer cells in vitro and in vivo. SIO-AF750-BBN demonstrated a significant photoacoustic contrast enhancement in tumor tissues, i.e. 3 and 5-fold as compared to the receptor-blocking control group and the peptide analog AF750-BBN without 
attachment to a nanoparticle, respectively, after intravenous tail vein injection in tumorbearing mice. In addition to bombesin agonist as the targeting moiety, bombesin antagonist is also explored. A series of bombesin antagonists with three different NIRF dyes and three pharmacokinetic modifiers were synthesized and characterized. Of those, three bombesin antagonist analogs, AF750-G-pip-Sta-BBN, AF750-GSG-Sta-BBN, and AF750-6Ahx-Sta-BBN, exhibited high NIRF imaging efficacy, high binding specificity and affinity, and antagonism to human PC-3 prostate cancer cells both in vitro and in vivo.

In conclusion, this Ph.D. work addresses the issues of discovery and development of novel site-specific and multi-modality molecular probes for cancer targeted imaging. USPIO(Cy7.5)-BBN displayed the capacity to generate imaging contrast enhancement for prostate tumors in living bodies on MRI/NIRF. The SIO-AF750-BBN demonstrated tumorspecific photoacoustic signal enhancement, as well as near-infrared fluorescent imaging enhancement after intravenous administration of this agent in human prostate cancer animal model. Finally, AF750 labeled bombesin antagonist analogs were developed and demonstrated to have a high binding affinity and selectivity to the GRPr and a desirable in vivo bio-distribution profile, indicating their high potentials to be translated to clinical use for imaging-guided surgery and therapy. 


\section{Chapter I. Introduction}

\section{Motivation}

Cancer is a large family of diseases that involve abnormal cell growth and potentials to metastasize. Most cancer has five stages. ${ }^{156}$ Stage 0: cancer in situ, abnormal cells are present. Not cancer, but it may become cancer. Stage 1: formed cancer or tumor has not grown deeply into nearby tissue and hasn't spread to lymph nodes. Stage 2 and 3: large cancers or tumors have grown deeply into nearby tissue, and it has spread to nearby lymph nodes. Stage 4 : cancer has metastasized to a distant part of the body. Although most early-stage cancers (Stage 0 and 1 ) are treatable, the optimal time of therapy is frequently missed because of unobvious symptoms of earlystage cancer and difficulties in early stage screening or diagnosis. Since most cancer deaths were due to metastasis (Stage 4), the improvement of early-stage cancer detection is critical for saving cancer patients' lives.

Routine screening of cancer may involve physical examinations, blood tests or urine tests, but neither of them can lead to a diagnostic decision. Notwithstanding that, due to the limited accuracy of these methods, misdiagnosis frequently happens that leads to over treatment or insufficient treatment. For example, prostate cancer has been over-treated in a significant number of cases after the introduction of prostate- 
specific antigen (PSA) test. ${ }^{1}$ Due to the defects of these methods, other diagnostic methods, like medical imaging and endoscopy, are preferable in most cases.

To improve medical imaging accuracy, molecular imaging technique was introduced at the end of the last century. Unlike the traditional imaging methods, molecular imaging techniques rely on the use of molecular imaging probes, whose categories include nanoparticles, radiotracers or fluorescent dyes. These imaging probes can approach to specific targets for imaging particular cellular functions and molecular processes. In molecular imaging procedures, the molecular imaging probes enable the visualization, characterization, and quantification of molecular abnormalities via detecting magnetism (magnetic resonance imaging or MRI), optical signals (bioluminescence and fluorescence), acoustic waves (ultrasound), radiation signals (nuclear medicine techniques), etc. On account of high accuracy, sensitivity and specificity, molecular imaging is believed to play a pivotal role in decreasing cancer mortality and improving prognosis in the new era.

My research interests lie in improving the sensitivity and specificity in the diagnosis of early-stage prostate cancer by molecular imaging. Prostate cancer can be screened with the prostate-specific antigen (PSA) test, digital rectal examination (DRE) and biopsy. These techniques indeed led to a decreasing incidence of advanced disease and death of prostate cancer in the past decades, but they also resulted in a huge number of cases being over-diagnosed and over-treated. In fact, a lot of people with low-risk tumors or potential prostate cancer risks do not need aggressive or invasive 
treatment. ${ }^{2}$ Overdiagnosis, and overtreatment in prostate cancer carries a significant, long-term, and adverse effect on quality of life. On the other hand, the screening techniques themselves are sometimes invasive and may bring about side effects like erectile dysfunction or urinary incontinence. ${ }^{2}$ Therefore, medical imaging techniques, such as computed tomography (CT), Positron emission tomography (PET) and magnetic resonance imaging $(\mathrm{MRI})$, are commonly used as complements or alternatives to the screening techniques for prostate cancer diagnosis.

\section{Objectives and Rationale}

The primary objective of this Ph.D. work is to develop novel multimodal molecular imaging probes, via nanotechnology and peptide conjugation chemistry, for site-specific and sensitive imaging of human prostate cancer cells. The pre-clinical examinations, including in vitro tests and in vivo tests, were performed to characterize the receptor binding affinity and selectivity and imaging efficacy of the developed novel compounds.

Bombesin (BBN), a 14-amino-acid neuropeptide, has a very high affinity to BB2 or gastrin releasing peptide receptors (GRPr) that are overexpressed in a variety of cancer types, including prostate cancer, breast cancer, pancreatic cancer and small cell

lung cancer. Therefore BBN could serve as targeting vector for tumor imaging. ${ }^{109,110}$ In fact, a lot of efforts have been made on synthesizing radio-labeled BBN derivatives for specifically targeted molecular imaging and radiotherapy. ${ }^{68-80}$ In this work, an hypothesis 
was made that the BBN derivatives associated ultrasmall paramagnetic iron oxide (USPIO) nanoparticle could also possess a specific and high binding affinity for targeting prostate cancer.

Particularly in the thesis, two BBN agonist-labeled nanoparticle multimodal imaging contrast agents with specific coating materials and strategies were described. The first, casein coated ultra-small (with a $5 \mathrm{~nm}$ core) iron oxide nanoparticle with NIRF dyes labeled bombesin (USPIO(Cy7.5)-BBN) nanoparticle was developed as a tumorspecific MRI and NIRF dual imaging modality contrast agent. The second, silica coated iron oxide nanoparticle (with a $10 \mathrm{~nm}$ core) decorated with NIRF dyes labeled bombesin (SIO-AF750-BBN) was developed as a multimodal PAI/NIRF/MRI imaging agent, with an emphasis on photoacoustic imaging (PA) enhancement to increase sensitivity and resolution for detection of tumor and circulating tumor cells. In addition to the bombesin agonist as the targeting moiety, bombesin antagonist was recently considered to be a good candidate peptide for targeting GRPr, elucidated by Cescato et al? Bombesin antagonist can potentially prevent the side effects caused by bombesin agonist. ${ }^{7-10}$ This thesis explored bombesin antagonists with different combination of NIRF dyes and pharmacokinetic modifiers to select highly effective cancer-specific bombesin antagonist NIRF analogs with a desirable bio-distribution profile for potential translation to clinical use for imaging-guided surgery and therapy.

MRI offers unique advantages of high resolution with a non-invasive operation, and it is currently implemented to help determine prostate cancer stage by evaluating 
how far the cancer has spread, but not in early stage prostate cancer diagnosis since it is restricted by its relatively low sensitivity. To enhance its application, significant efforts have been made in developing better MRI contrast agents. ${ }^{3-5}$ In Chapter 3, a near infrared fluorescent (NIRF) dye labeled superparamagnetic iron oxide (SPIO) nanoparticle with a bombesin peptide as the targeting moiety was designed for prostate cancer imaging. The involvement of fluorescent dye was to take advantage of the high sensitivity of NIRF imaging to complement MRI. NIRF imaging is an imaging procedure that constructs $2 \mathrm{D}$ or $3 \mathrm{D}$ images by collecting fluorescent signals from administrated fluorescent dyes in the body. It features an outstanding sensitivity, low operation costs and time periods, and is applied in image-guided surgery pre-clinically and clinically. ${ }^{6}$ On the other hand, molecular imaging agent with targeted delivery attracted people's attention over the advantages of enhanced tissue to background ratio (TBR) and cutdown of required dosage. Thus, Bombesin (BBN), as a targeting peptide to various cancers that overexpress gastrin releasing peptide receptor (GRPr), was conjugated with USPIO nanoparticles and NIRF dye to further improve the sensitivity and tumor to background ratio in NIRF/MRI.

In Chapter 4, my ultimate objective is to develop a targeted imaging probe for the detection of circulating tumor cells (CTCS), since the existence of a large number of CTCS is a significant sign for cancer metastasis. CTCs are a group of cells that fall from the cancerous tumors into the bloodstream and have a possibility to move and form a new metastasis. Population growth of CTCs in the bloodstream is a crucial sign for metastasis, and early detection of CTC offers an unprecedented opportunity to diagnose 
cancer before metastasis. Moreover, tracking the number and distribution of CTCS enables the monitor of the response of patients to therapies. ${ }^{11,12}$ The limitation of current techniques is the undesirable detection sensitivity, since only several CTCs exist in $1 \mathrm{ml}$ blood, with around $5 \times 10^{9}$ erythrocytes and $1.0 \times 10^{7}$ leukocytes. ${ }^{13}$ Currently, capture and analysis of CTCS are performed by a series of independent procedures including CTCs detections and enrichment using microfluidic CTC chips, magnetic particles, microfiltration, dielectric separation and flow cytometry, and CTCs isolation by a physically based method or biologically based method. The whole procedure is very time consuming, and therefore, is adverse to timely treatment. In this project, we aimed to develop a silica coated iron oxide nanoparticles as a photoacoustic imaging probe with a bombesin agonist as the targeting vector to CTCs, where our objective is to explore the possibility of directly detecting CTCs in peripheral blood in cancer patients with the developed nanoparticle. In the principle of PA imaging, the tissue of interest is excited by pulsed laser to emit an ultrasonic wave that is collected by an ultrasonic transducer. Thus, photoacoustic imaging is very meaningful in that it integrates high selectivity of optical excitation with the high signal to noise ratio inherent in ultrasound propagation. ${ }^{11}$ In the first phase of this project, I conducted the synthesis of the nanoparticles and its examinations in in vitro and in vivo studies in PA/NIRF imaging and gained encouraging outcomes that are helpful to extend the application of PA imaging to tumor detections.

In Chapter 5, bombesin antagonist, as a newly emerged targeting peptide, was conjugated with NIRF dyes and spaced with several different linkers for exploring its 
targeting and imaging efficacy to prostate cancer. Bombesin antagonist has a similar behavior to the well-developed bombesin agonist except for the manner of cellular uptake in that bombesin antagonist only binds on the outer surface of the cancer cells, while bombesin agonist can be internalized into cells. The nanoparticle may be too big to stay on the surface of the cancer cells and would likely move away from its binding sites by the outer flow. Thus, we used NIRF dyes to conjugate with bombesin antagonist to form small molecules for the targeting tests. NIRF is a rapid imaging modality and is advantageous of high sensitivity, so it can be an adaptable strategy for imaging-guided therapy or surgery of prostate cancer.

\section{Organization of the Dissertation}

This Ph.D. thesis is organized as follows: Chapter 1 describes the introduction to this report. Chapter 2 provides the background knowledge of imaging techniques. Chapter 3 describes the studies regarding casein coated iron oxide nanoparticle with site-specific targeting ability to prostate cancer. Chapter 4 describes the studies about developing fluorescent dye labeled silica coated iron oxide nanoparticle with bombesin as a photoacoustic imaging and near-infrared fluorescent imaging probe to prostate cancer and circulating tumor cells. Chapter 5 describes the studies concerning the near infrared

fluorescent dye labeled bombesin antagonist to target prostate cancer. Chapter 6 summarizes the main outcomes of the research and proposes outlooks for future work and relevant areas. 


\section{Chapter II. Background}

\section{Nanoparticles based molecular imaging and targeting strategies}

Molecular imaging can be used for early detection of tumor and real-time monitoring of therapeutic response, where the development of imaging probe plays a pivotal role in the improvement of molecular imaging. Typical imaging probes in molecular imaging are radiolabeled molecules, nanoparticles, and antibody, etc.

Nanotechnologies enable researchers to manipulate matter at the nanoscale (from $1 \mathrm{~nm}$ to $100 \mathrm{~nm}$ ). The materials at the nanoscale can display some new and unusual properties in chemical reactivity, optical behavior, magnetic behavior, etc. In the field of medicine, nanoparticles are significant candidates for disease treatment, drug delivery, molecular imaging, etc, as the Figure 2.1 shows. For example, gold nanoparticles can be used in photothermal therapy as a strong optical absorber. ${ }^{14}$ Iron oxide nanoparticle as an MRI contrast agent has been widely studied, and it is also studied in hyperthermia cancer treatment since it's able to release massive heat in fast alternating magnetic field. ${ }^{15-17}$ Quantum dots can be applied as imaging probes for NIRF imaging. On the other side, enhanced permeability and retention (EPR) effect is a particular property of nanoparticle, by which nanoparticle can take advantages of the deficient angiogenesis of tumor to increase the accumulation at the tumor site. ${ }^{18,19}$ Due 
to this effect, nanoparticles have been vastly studied for cancer treatment or sitespecific imaging. ${ }^{20,21}$

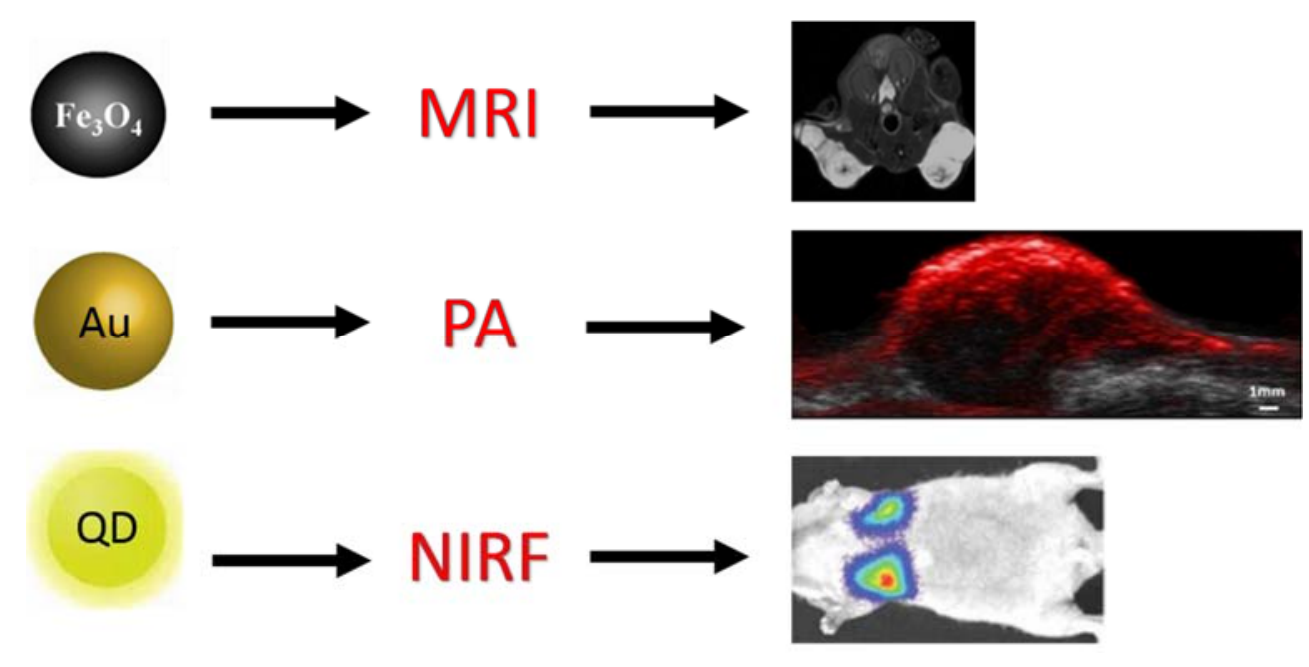

Figure 2.1 Applications of nanotechnologies in the field of medical imaging.

For a nanoparticle, a passive transportation may be realized by aforementioned EPR effect, but there exist some limitations. For example, unlike targeted delivery that can offer receptors mediated endocytosis for nanoparticles, EPR effect doesn't confer a pathway for nanoparticles to be rapidly internalized into tumor cells. On the other side, the degree of tumor vascularization and porosity of tumor vessels can vary much with tumor types. The neo-vasculature of early stage cancer is not rich, bringing about a difficulty for nanoparticles to get close to cancer cells. Thus, besides the passive transportation, installing "radar" onto the imaging probe is of particular importance to direct them to the disease site. In recent years many efforts have been made on developments of targeting vectors that can enable nanoparticles to have high binding affinities for the target, long retentions at targeting tissue, quick removals from 
background tissues, desirable bio-safeties, bio-stabilities, and simple protocols to synthesize.

General targeting moieties include antibodies, peptides, small molecules, or aptamers. Antibody-based targeting moiety usually has outstanding binding affinity and selectivity for the target. They are, however, too large in dimension, too expensive for manufacturing, having a large variation from batch to batch and having potentials to induce immunogenic responses. About its applications, for example, a single chain antiprostate stem cell antigen antibody (scAbP-SCA) has been used as a targeting moiety for prostate cancer imaging and therapy. The labeled superparamagnetic iron oxide nanoparticle distinctly darkened prostate cancer more than that in the control group without SCAbP-SCA. ${ }^{22}$

Peptide-based targeting moieties have small dimensions, low immunogenicity, and simple procedure of manufacturing. ${ }^{23}$ But they have some drawbacks, including short blood circulation time, relatively low binding affinity and susceptibility to proteolytic cleavage. ${ }^{24}$ Peptide based targeting strategy has been vastly explored and some of them are in clinical trials. Arg-Gly-Asp(RGD) peptide strongly binds to $\alpha v \beta 3$ receptor that is overexpressed on activated and proliferating endothelial cells during tumor angiogenesis but not in normal tissues. Compounds utilizing the RGD motif, like 18F-galacto-RGD and 18F-ah111585 are currently under clinical trials. ${ }^{25,26}$ In another example, Zhang et al. developed a magnetic/optical nanoprobe to specifically target brain tumor across blood-brain barrier (BBB) with chlorotoxin (CTX), a 36 amino acid 
peptide that is capable of permeating across BBB and targeting to tumors of neuroectodermal origin. ${ }^{29} \mathrm{MRI}$ and NIRF experiments confirmed the selectivity and specificity of CTX labeled nanoparticle to brain tumors. Our group has studied Bombesin extensively in the past decade. ${ }^{81,111}$ Bombesin, a 14 amino acid peptide, can target to gastrin releasing peptide receptors that are overexpressed in a variety of human cancers including prostate cancer, breast cancer, colon cancer and small cell lung cancer but not in normal tissues except for pancreas. Many bombesin analogs with radionuclides, like ${ }^{64} \mathrm{Cu}-\left[\mathrm{DOTA}-\mathrm{Lys}^{3}\right]-\mathrm{BBN}^{27}$ and ${ }^{18} \mathrm{~F}-\left[\mathrm{FB}-\mathrm{Lys}^{3}\right]-\mathrm{BBN}^{28}$, are considered to be in a clinical translation. AF680-GGG-BBN[6-14] is promising for lymph node metastases detection in prostate cancer.

A majority of small molecule-based targeting moieties is folate (folic acid). Folate is easy to synthesize at a low cost. It has a high binding affinity to folate receptors overexpressed by tumor cells. Folate labeled imaging probes developed very fast, and several compounds are currently under clinical trials, like ${ }^{111}$ In-DTPA-folate, ${ }^{99} \mathrm{mTc}$-folate conjugate (EC20), folate-linked fluorescent hapten (EC17), and diacetylvinylblastine hydrazide-folate conjugate (EC145).

Aptamer-based targeting moiety is advantageous of high binding affinity, low immunogenicity and little variations from batch to batch. Its defect is rapid blood clearance, caused by nuclease degradation. Aptamers are a group of small singlestranded DNA and RNA sequences (20-100 bases) that can bind to targets by folding into well-defined three-dimensional structures. Currently, aptamers have been clinically 
used in therapy for neovascular macular degeneration. A variety of aptamer based imaging probes utilized in MRI, optical imaging, ultrasonic imaging, CT imaging and nuclear imaging are in pre-clinical test. For example, Wang and their group successfully used A10 aptamer to conjugate with SPIO to target PSMA on prostate cancer cells with high specificity. ${ }^{30} \mathrm{Hwang}$ et al. combined a cobalt-ferrite nanoparticle with fluorescent rhodamine, 67Ga and AS1411 aptamer to target against nucleolin-expressing tumor cells for multi-modality of $\mathrm{MRI}$, optical imaging, nuclear imaging. ${ }^{31}$ The in vitro and in vivo outcomes confirmed the specificity and high binding affinity of the compound.

\section{Principle of MRI and MRI Contrast Agent}

\section{Physics behind Magnetic Resonance Imaging}

Magnetic resonance imaging (MRI) is currently one of the most widely used and robust diagnostic tools. The mechanism of MRI is based on non-zero self-spin of atoms in a magnetic field. Most MRI procedures rely on signals from protons because protons outnumber all other atoms largely in human body and protons have $1 / 2$ spins that form large magnetic momentums. The nuclear magnetic moment is related with spin angular momentum by:

$$
\vec{\mu}=\gamma \text { 国, }
$$
Where ?ils spin angular momentum, $\vec{\mu}$ represents magnetic momentum, and $\gamma$ is 
found $\gamma / 2 \pi$ of various elements: For ${ }^{1} \mathrm{H}, \gamma / 2 \pi$ is $42.58 \mathrm{MHz} / \mathrm{T}$, and for ${ }^{13} \mathrm{C},{ }^{17} \mathrm{O},{ }^{19} \mathrm{~F},{ }^{23} \mathrm{Na}$, ${ }^{31} \mathrm{p}, \gamma / 2 \pi$ are $10.7 \mathrm{MHz} / \mathrm{T}$, $5.8 \mathrm{MH} z / \mathrm{T}_{\text {dively. }} 40.1 \mathrm{MHz} / \mathrm{T}$, $11.3 \mathrm{MHz} / \mathrm{T}$, and $17.2 \mathrm{MHz} / \mathrm{T}$,

The magnitude of spin angular momentum is given by:

$$
\mid \text { 国 } \mid=\hbar \sqrt{\text { ? }(\text { ? }+1)}
$$

This equation is derived from quantum mechanics. ? represents the nuclear spin number, which is unique for each element. For ${ }^{1} \mathrm{H}, \mathrm{I}=1 / 2$. In the equation, $\hbar=h / 2 \pi$, where $\mathrm{h}$ is Planck's constant, equal to $6.63 \times 10^{-34} \mathrm{Js}$. In the absence of the external magnetic field, the magnetization of protons can randomly flip directions, and the net magnetization appears to zero. In a magnetic field, however, protons will align either parallel as at a low energy state or anti-parallel as at a high energy state. The distribution will obey Boltzmann distribution, and thus, a majority of protons will be in low energy state and the direction of net magnetization will align parallel with the magnetic field. The formation of two different energy states is called Zeeman splitting.

In classical physics, because of the energy difference, each atom will rotate around the magnetic field with an angle while moving forward in the presence of an external magnetic field. This motion is called Larmor procession, and the frequency of rotation is called Larmor frequency. The frequency is related to the magnetic field strength and gyromagnetic ratio:

$$
\omega=\gamma B_{0}
$$


The energy difference between parallel spin and antiparallel spin is:

$$
\mathrm{E}=\gamma \hbar B_{0}
$$

\section{Magnetic Resonance Imaging Procedure}
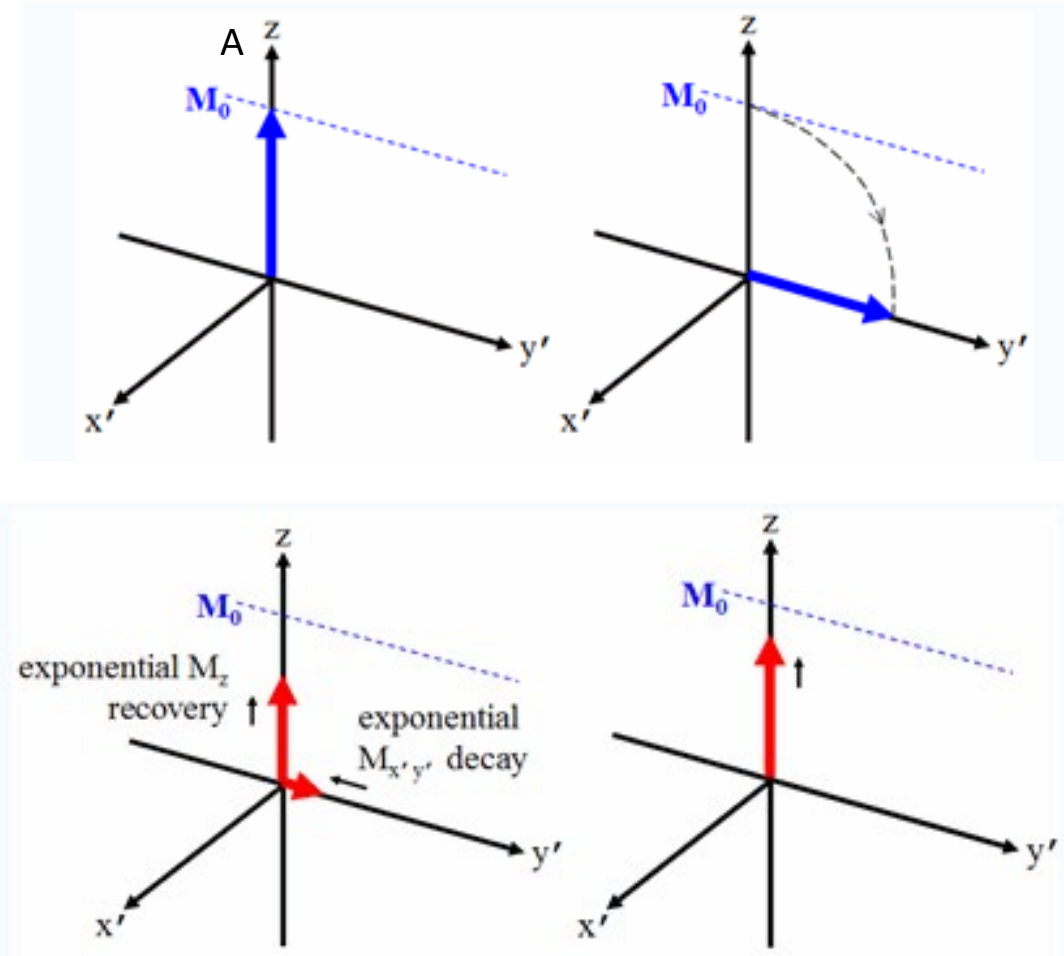

Figure 2.2 Influence on net magnetization by RF pulse

A magnetic field gradient $\left(B_{0}\right)$ is a variation in the magnetic field with respect to different positions, and $\mathrm{B}_{0}$ 's function is to record positions of excited protons. To achieve information of protons' spins in tissues, one radio frequency (RF) pulse is applied, which is also named as the B1 field. In order to render protons absorb the RF pulse, the frequency of RF pulse should match the energy difference between parallel spin and anti-parallel spin, namely, Larmor frequency. Data acquisition is realized by 
collecting magnetic waves released from excited protons. As displayed in Figure 2.1, initially, the net magnetization vector $M$ was $M_{0}$ and aligns with the external magnetic field $B_{0}$ in direction $z$. As RF pulse switched on, $M$ absorbs the energy of RF pulse and is flipped from $z$ to the transverse plane $(x-y)$, to generate a new magnetization at the $x-y$ plane, named $\mathrm{M}_{\mathrm{xy}}$.(Figure $2.1 \mathrm{~A}$, Figure $2.1 \mathrm{~B}$ ) $90^{\circ}$ is the largest flip angle, and any flip angles from $0^{\circ}$ to $90^{\circ}$ can be realized by tuning the RF pulse. When the RF pulse is switched off, the $z$ component of $M$ will recover to $M_{0}$ (Figure 2.1D), and the $x-y$ component of $\mathrm{M}$ will decay to zero (Figure 2.1C). RF relaxation was dependent on the parameters called relaxation time by the Bloch equation:

$$
\begin{aligned}
& { }^{\square \vec{M}}=\gamma \vec{M} \times B(t)+{ }^{1}(M-M) \hat{z}+{ }^{1} \vec{M} \text {, }
\end{aligned}
$$

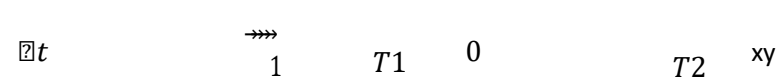

[]

In this equation, $T_{1}$ means the time needed to recover to $63 \%$ longitudinal

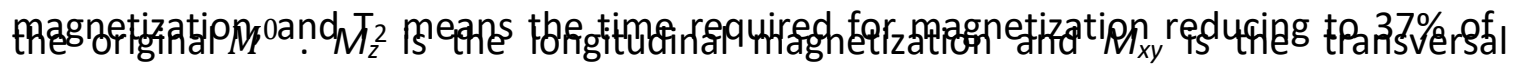
magnetization. $M_{x y}$ and $M_{z}$ can be calculated by equations:

$$
\begin{aligned}
& -\frac{t}{-} \quad 0 \quad-t / T \\
& M_{\text {回 }}(t)=M_{0}\left(1-T_{1}\right), M_{\text {回回 }}(t)=M_{\text {回回 }} e \quad 2 \text {, } \\
& e
\end{aligned}
$$

In MRI imaging, the contrast between different tissues in images is dependent on specific values of $T_{1}$ and $T_{2}$ of protons from different tissues. Accordingly, there are $T_{1}$ weighted $M R I$ image and $T_{2}$ weighted $M R I$ image.

In the process of data acquisition, four basic types of MRI signals that may apply: 
free induction decay (FID), gradient echo (GRE), spin echo (SE) and stimulated echo. For 
free induction decay, when a $90^{\circ} \mathrm{RF}$ pulse is given, net magnetization $\mathrm{M}$ will be flipped into the $x-y$ plane (transverse plane). A receiver coil placed in the transverse plane will be induced to have a voltage, and the magnitude of voltage is relating to the magnetization in the transverse plane. As the RF pulse switched off, energy will be released in the form of RF pulse that is to be collected by the receiver coil. The signal produced by the transversal magnetization decay is named free induction decay. While for spin echo, another $180^{\circ}$ RF pulse is applied after the $90^{\circ}$ RF pulse, resulting in the magnetization to rephase and an echo is produced as the signal. In the process of producing gradient echo, applied RF pulse flips magnetization by an angle $\alpha$, and then a gradient pulse is applied to rephase spins.

Although $\mathrm{MRI}$ is a robust imaging tool and is widely used in clinics, such as neurology, cardiology, and cancer, it may be not very satisfying to exhibit the difference of lesions and normal tissues, because the relaxation time of them may be very close. To make the signals to be more distinguishable, contrast agents were developed and nowadays, $30 \% \mathrm{MRI}$ procedures rely on the use of contrast agents.

In a human body, MRI contrast agents interact with surrounding water protons to shorten relaxation time so that the brightness of tissues of interest can be tuned. According to the principle of actions, MRI contrast agents are classified as T1 and T2 contrast agent. T1 contrast agents, that significantly influent $\mathrm{T}_{1}$ relaxation, are mainly gadolinium based agents. Some of them, like Ominiscan, have been approved by FDA and widely used in clinics for brain or spinal MRI scan. T2 contrast agents, which 
extensively affect $T_{2}$ relaxation, are mostly iron oxide nanoparticle based contrast agents, and recently new types of nanoparticle were designed which also show excellent contrast enhancement ability, like FePt nanoparticle, $\mathrm{Fe}_{5} \mathrm{C}_{2}$ nanoparticle. ${ }^{7}$ All these nanoparticles feature superparamagnetism (Figure 2.2), a special property for particles to recover to random directions of magnetism, instead of maintaining partial magnetism to induce new aggregations, after the cancel of external magnetic field. After intravenous injection of the T2 contrast agent into the human body, with the T2 relaxation of surrounding water protons to be facilitated, the signal of adsorbing tissue will be tuned to be darker in the reconstructed T2 weighted image. Examples of FDA approved T2 contrast agents are Feridex (recently discontinued by the manufacturer) and Resovist. Feridex can be intensively accumulated in the liver after administration, and since the hepatic region with lesion has lost the function of absorbing Feridex, the lesion area will display a brighter signal than surrounding normal hepatic tissues.
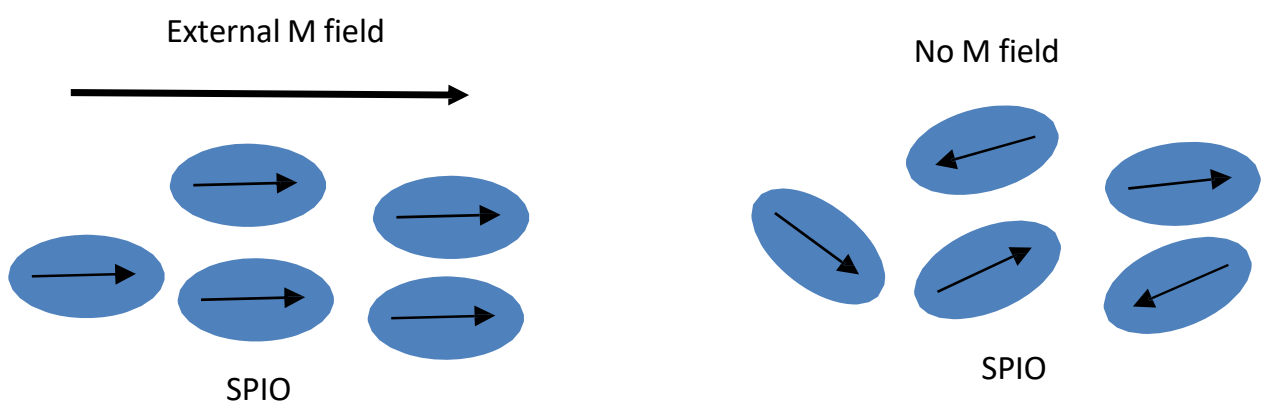

Figure 2.3 Superparamagnetism of iron oxide nanoparticle 
However, the extension of iron oxide nanoparticle's application in MRI contrast enhancement is limited by a short blood circulation time. For those iron oxide nanoparticles with size up to $150 \mathrm{~nm}$ or more, including Feridex, they will be readily sequestered by macrophages in the liver (Kupffer cells) and spleen ${ }^{32}$, which is lower than ideal for medical imaging. Scientists have been devoted to comprehending the mechanism of this effect and are trying to minimize it. Up to date, surface charge and particle size have been found to potentially affect the circulation time and accumulation of nanoparticles in the liver. Basically, positively charged nanoparticles readily bind to negatively charged cellular membrane of macrophages, while negatively charged nanoparticles may experience endocytosis via protein mediated phagocytosis and diffusion. ${ }^{33}$ A neutrally charged surface of nanoparticles was reported to show a reduced phagocytic uptake and is preferable for imaging and drug delivery. ${ }^{34,35} \mathrm{~A}$ fast removal of iron oxide nanoparticles with size more than $200 \mathrm{~nm}$ will occur, leading to an accumulation of nanoparticles in the liver, while small iron oxide nanoparticles will be rapidly filtered out by the kidney. Thus, iron oxide nanoparticles with dimensions ranging from $10 \mathrm{~nm}$ to $150 \mathrm{~nm}$ will be relatively fit for imaging. In addition to particles' sizes and surface charges, modification of nanoparticles with targeting moieties offers a way to deliver without much loss for imaging objectives. 


\section{The Principle of Photoacoustic Tomography and Photoacoustic Contrast Agent.}

Photoacoustic tomography (PAT) is an increasingly popular imaging modality based on photoacoustic effect. The photoacoustic effect, which was initially discovered by Alexander Graham Bell in 1880, means the valid evaluation of absorbed electromagnetic energy on matters by acoustic measurement. In PAT, one laser exposed to human body will be absorbed by tissues and converted into heat, leading to transient thermoelastic expansion and simultaneously pressure is released via a wideband ultrasonic emission. Collecting the ultrasonic waves through an ultrasonic transducer enables a way to analyze optical absorption by tissues. Because the optical absorption is closely related to physiological properties, ultrasonic information will reveal the physiologically specific optical contrast of different tissues. According to these basics, a $2 \mathrm{D}$ or $3 \mathrm{D}$ image can be constructed to show the physiological environment of areas of interest. Since either scattered or unscattered optical waves can induce photoacoustic signals, a significant depth of photoacoustic wave emission can be made. Moreover, since ultrasonic scattering coefficient is 2 to 3 orders of magnitude less than optical waved, a high spatial resolution can be achieved. Thus, compared to other imaging modalities, PAT is the only one that combines excellent optical contrast and desirable ultrasonic spatial resolution. Additionally, PAT endogenous molecules, like hemoglobin or melanin, can exhibit a optical absorption, resulting in strong PAT signals in skin melanoma detection, blood oxygenation mapping and tumor angiogenesis monitoring. ${ }^{36}$ Current PAT applications include melanoma cancer screening, neonatal, and adult brain 
imaging, breast cancer imaging, prostate cancer imaging, dosimetry in thermal therapy, etc. Some of them are in clinical trial, and some of them have been translated into clinical practice.

As mentioned previously, PAT can be used for endogenous molecules imaging, like oxyhemoglobin, deoxyhemoglobin, water, or melanin. A group of contrast agents, such as organic dyes, nanoparticles, have been developed to extend PAT applications in more contexts. Indocyanine-green (ICG) is an FDA approved fluorescent dye that can be used for both fluorescence imaging and PAT contrast enhancements. Saeid Zanganeh et al. conjugated ICG with single-walled carbon nanotube to enhance PAT detection of breast cancer mice model and a positive outcome was achieved. ${ }^{37}$ Jeon et al. invented a methylene blue micro bubble that utilizes clinically approved methylene blue to initiate a new type of PAT contrast agents with extensively high PAT enhancement. ${ }^{38}$ There are also many other dyes under test, such as AF750, BHQ3, QXL680, etc. ${ }^{39}$

Besides dyes, nanoparticles are another group of PAT contrast agents, featuring inherent optical absorption, surface plasmon resonance (SPR), and surface modification potentials. The most typical example is gold nanoparticles. Gold nanoparticles have strong inherent SPR effect. The photoacoustic properties of gold nanoparticles are found to be highly correlated to dimensions and shapes. For example, original gold nanosphere has a typical absorption at $520 \mathrm{~nm}$, which can be tuned to $600 \mathrm{~nm}$ when the particle's size is up to $100 \mathrm{~nm} .{ }^{40}$ To obtain different photoacoustic properties, gold nanoparticles were developed with various complicated shapes, such as gold 
nanorods ${ }^{41}$, gold nanoshells ${ }^{42}$, gold nanoprisms ${ }^{43}$, gold nanocages ${ }^{44}$, gold nanostars ${ }^{45}$, and gold nanovesicles ${ }^{46}$. They displayed different absorption coefficients, absorption wavelengths, and engineering potentials. Compared to organic dyes, which can only circulate in the human body for several minutes before excretion by the kidney, gold nanoparticles can stay in the human body for a longer time, especially as prolonged retention time in tumor through enhanced permeability and retention (EPR). Moreover, gold nanoparticles display several orders of magnitude higher of absorption coefficient than dyes. Up to date, possibilities of gold nanoparticles as PAT contrast agents have been explored for human breast tumor ${ }^{47}$, melanomas ${ }^{48}$, and brain tumors ${ }^{49}$. Another exciting exploration with gold nanoparticles is the detection of CTCs, whose feasibility has been demonstrated by an experiment where detection limitation is up to ten breast cancer cells with a PA flow cytometry system ${ }^{50}$. The possibility of detecting circulating prostate cancer cells and melanoma cells has also been substantiated with gold nanospheres in a static system ${ }^{51,52}$. Other potential applications for gold nanoparticles include lymph node mapping for cancer cell metastasis, atherosclerotic plaques imaging, brain functional imaging, and PAT guided therapy. As studies about PAT contrast agents continue to develop, silver nanoparticles, quantum dots, and superparamagnetic iron oxide nanoparticles are also found to have potentials as PAT contrast agents. ${ }^{21,53,54}$ 



\section{NIRF Imaging and Imaging Probes.}

Near-infrared fluorescence (NIRF) imaging features a high sensitivity and multidetection capability, can be clinically applied in intra-operative procedures and imaging guided therapies. Moreover, high sensitivity enables the use of NIRF imaging to monitor pathological process and cell's activities at a molecular level. Manipulation of NIRF imaging relies on the use of exogenous NIR fluorophores that emit in the near infrared spectral region (600-900nm), bringing about a desirable penetration depth (about several centimeters) into the human body without substantial interference from tissue auto-fluorescence or absorption by water, lipid, and hemoglobin at a small wavelength. In this range, the fluorescent dye that emits at a longer wavelength is preferable since they will penetrate deeper into matters. Most organic dyes are susceptible to photobleaching and are not hydrophilic. Thus, only a small group of organic dyes are qualified as NIRF imaging agents. ICG is currently the only fluorophore that is clinically approved and has been used intraoperatively in coronary, neurosurgical, vascular surgeries, and for non-invasive assessment of superficial perfusion. ${ }^{55}$ However, its application in NIRF imaging is limited by the low quantum efficiency, and no functional group for labeling targeting moieties. Other dyes, like rhodamine dyes, bodipy based dyes and squaraine based dyes are under developments or pre-clinical trials. Compared to organic dyes, quantum dots are advantageous of their excellent extinction coefficients, easily tunable emission wavelength, and high endurability of photobleaching. ${ }^{56}$ The primary defect of quantum dots is the relatively high toxicities, but coating strategies can be developed to improve the bio-safety. On the other hand, 
the aforementioned dyes can be covalently or non-covalently conjugated with nanoparticles like gold nanoparticles or silica nanoshells to potentially be conferred with enhanced biocompatibility, lower photobleaching, and more loading space for targeting moieties.

Although NIRF imaging has a limited resolution, it offers a unique advantage of extremely high sensitivity that complements properties of MRI or PAT. The multimodality imaging integrates desirable resolution, sensitivity, good penetration depth and anatomical display and will more fit the requirements of personalized diagnosis and therapy. 


\section{Chapter III. Bimodal MRI/Fluorescence Imaging Contrast Agent Targeting Human Prostate Cancer}

\section{Introduction}

Prostate cancer is the second leading cause of cancer death in U.S. for men. In 2015 , there are 220,800 new cases and 27,540 new deaths from prostate cancer. Although 5 -year relative survival rate is up to $100 \%$ of patients diagnosed with localized or regional prostate cancer, this rate will dramatically drop to $28.2 \%$ when metastasis occurs. ${ }^{57}$ Thus, robust diagnostic techniques for non-metastasis cancer are critically needed for the reduction of recurrence rate and the improvement of prognosis. Current techniques, including rectal exam, PSA blood test, and biopsy, are insufficiently accurate for the early detection and diagnosis of prostate cancer, frequently bringing about misdiagnosis or over-treatment. ${ }^{1,58}$ Thus, cancer-specific molecular imaging probe for prostate cancer detection and diagnosis with high sensitivity and selectivity is urgently needed.

Superparamagnetic iron oxide nanoparticle (SPIO) has been widely applied in the field of biomedicine, such as the contrast agent in magnetic resonance imaging (MRI) and magnetic hyperthermia. SPIO possesses a lot of advantages, including non-toxicity, biodegradability and versatility for engineering. ${ }^{59-61}$ In MRI applications, SPIO enhances contrast in $\mathrm{T} 2$ weighted MRI images by extensively reducing the transverse relaxation 
time (T2) of water protons in absorbing tissues that could be liver, spleen or tumors. ${ }^{62}$ Conventional SPIO is limited in cancer-related applications, due to its relatively big size (hydrodynamic diameter is usually larger than $50 \mathrm{~nm}$ ) and the aggregating tendency, leading to a rapid capture by macrophages and a quick collection in liver and spleen. ${ }^{63,64}$ Furthermore, since the conventional SPIO is not specifically directed to the disease site, the insufficient concentration of SPIO at the disease site is unfavorable to generate good contrast in MRI images.

For all of these reasons, we are devoted to developing an ultra-small $(5 \mathrm{~nm})$ superparamagnetic iron oxide nanoparticle (USPIO) that is capable of selectively targeting to prostate cancer. The idea of designing an ultra-small nanoparticle is aimed to elongate the circulation time, even though it has to compromise for a reduction of MRI $r 2$ relaxivity due to the principle that $r 2$ relaxivity decreases as the nanoparticle's core size reduces. ${ }^{65}$ Casein, the major component of bovine milk protein, is a biocompatible and degradable material. ${ }^{66}$ Since casein is comprised of both hydrophobic and hydrophilic moieties and could be assembled by cross-link reactions, it can potentially be applied as the matrix to encapsulate hydrophobic USPIO for the improvement of the water solubility, biocompatibility, stability, and functionalization. ${ }^{66,67}$ Bombesin, a 14 -amino-acid neuropeptide, has a very high affinity to $\mathrm{BB} 2$ or gastrin releasing peptide receptors (GRPr) that are overexpressed in a variety of cancer types, including prostate cancer, breast cancer, pancreatic cancer and small cell lung cancer. In the past decades, a lot of efforts have been made on synthesizing radio-labeled BBN derivatives for specifically targeted molecular imaging and 
radiotherapy. ${ }^{68-80}$ In this work, an assumption was made that the $\mathrm{BBN}$ derivatives associated USPIO could also possess a specific binding ability for targeting prostate cancer with a high affinity. On the other side, near-infrared fluorescence imaging (NIRF), a promising candidate for early-stage cancer detection and screening ${ }^{56}$, features excellent sensitivity, short operation time and low costs. The fluorescent signals (600$900 \mathrm{~nm})$ of NIRF imaging probes, such as quantum dots and fluorescent dyes, could penetrate human bodies at a desirable depth. ${ }^{81}$ By acknowledging the relatively low sensitivity of MRI, fluorescent dyes were grafted to the USPIO to make the compound a bimodal MRI/NIRF imaging probe to integrate MRI's rich anatomical information, high spatial resolution with NIRF's high sensitivity. The final compound and its interaction with GRPr was schematically illustrated in Figure 3.1.

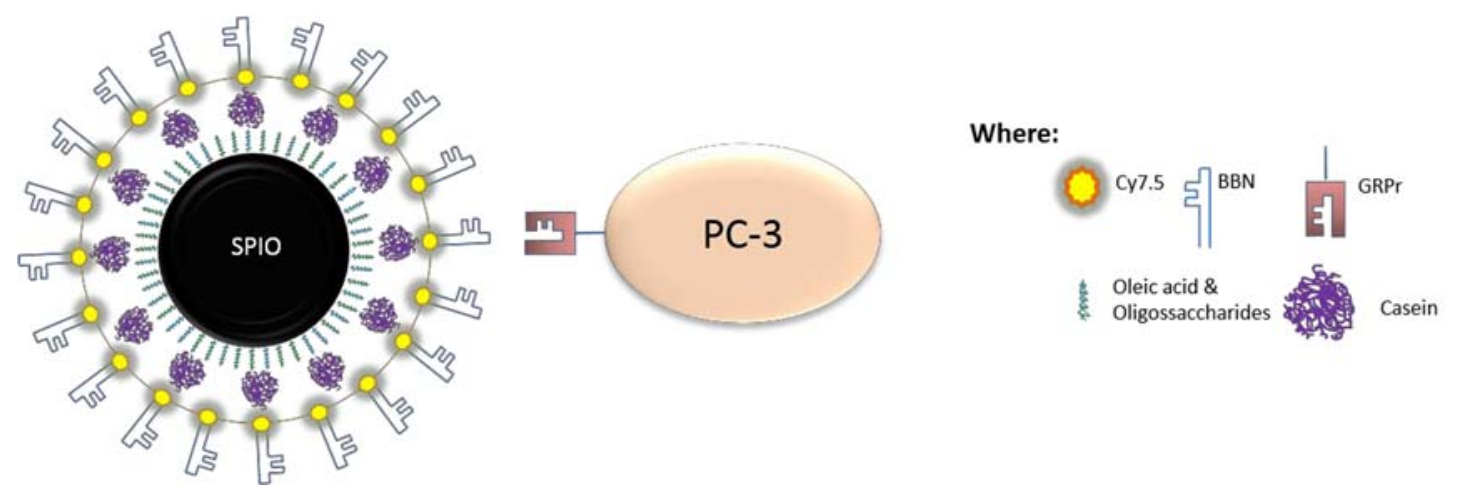

Figure 3.1 Schematic diagram of interaction between USPIO(Cy7.5)-BBN and prostate cancer cells

In this work, oleic acid coated USPIO was encapsulated to casein matrix and was loaded with agonist BBN (K-8AOC-QWAVGHLM-NH2) and Cyanine 7.5 (Cy7.5) molecules. Several techniques were employed to characterize this compound and afterwards, a 
cellular experiment in PC-3 cells and in vivo study in PC-3 xenograft models was performed to evaluate its binding specificity and affinity to prostate cancer and $\mathrm{MRI} / \mathrm{NIRF}$ imaging contrast enhancing capability in small rodent animals.

\section{Materials and Methods}

\section{Materials}

All solvents were either ACS certified or HPLC grade. Glucose, casein, glutaraldehyde, 1-ethyl-3-(3-dimethylaminopropyl)carbodiimide (EDAC) and hydroxylamine solution were purchased from Sigma-Aldrich. Dimethylformamide (DMF), sodium bicarbonate, trifluoroacetic acid (TFA), acetonitrile, acetate NHS ester, and sulfo-NHS were obtained from Thermo Fisher Scientific. Phosphate buffered saline (PBS) was purchased from Leinco Technologies (St. Louis, MO). Ethanol was obtained from Decon Laboratories (King of Prussia, PA). Iron oxide nanoparticle (5 nm core size, iron concentration $50 \mathrm{mg} / 2 \mathrm{~mL}$ ) with an oleic acid coating in chloroform was purchased from Ocean nanotech (San Diego, CA). Bombesin[1-14] peptides were purchased from American Peptides Co. Inc. (Sunnyvale, CA). ${ }^{125}$ I-Tyr4-BBN was obtained from NEN Life Science Inc. (Boston, MA). Cyanine 7.5 NHS ester was purchased from Lumiprobe (Hallandale Beach FL). Dialysis membrane with a molecular weight cutoff of $100 \mathrm{kDa}$ or 50kDa was purchased from Spectrum Laboratories, Inc (Rancho Dominguez, CA). All other solvents and reagents were obtained from Sigma-Aldrich (St. Louis, MO) and Thermo Fisher Scientific (Waltham, MA) or otherwise stated, and used as received. 


\section{Synthesis and purification of $\mathrm{Cy} 7.5-\mathrm{K}-8 \mathrm{AOC}-\mathrm{BBN}[7-14] \mathrm{NH}_{2}$}

The whole process of synthesis was illustrated in Figure 3.2.

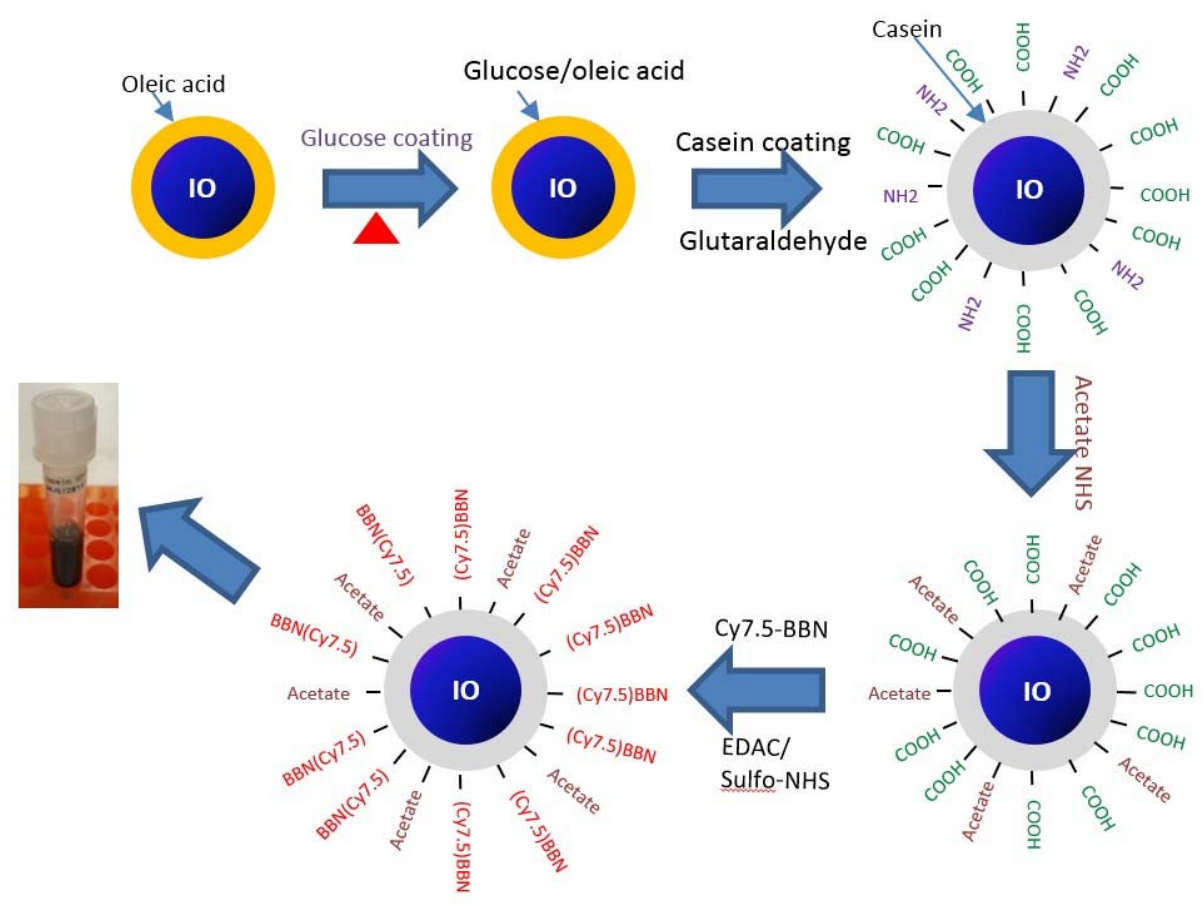

Figure 3.2 Schematic diagram of the synthetic process for the USPIO(Cy7.5)-BBN

Peptide Lys-8AOC-GIn-Trp-Ala-Val-Gly-His-Leu-NH2 (K-8AOC-BBN[7-14] $\left.\mathrm{NH}_{2}\right)$ was synthesized using conventional Fmoc solid-phase peptide synthesis (SPPS) and purified by high-performance liquid chromatography (HPLC) to a purity greater than $97 \%$ in house or by EZBiolab (Carmel, IN). The conjugation reaction of Cy7.5 to K-8AOC-BBN[714] $\mathrm{NH}_{2}$ was carried out at a 1: 1 molar ratio of Cy7.5: BBN. Briefly, approximately 1.2 mg of $\mathrm{H}_{2} \mathrm{~N}-\mathrm{K}-8 \mathrm{AOC}-\mathrm{BBN}[7-14] \mathrm{NH}_{2}$ peptide in $320 \mu \mathrm{L} 0.1 \mathrm{M} \mathrm{NaHCO}_{3}$ combined with 100 $\mu \mathrm{L}$ ethanol $\left(\mathrm{pH}=8.3^{\sim} 8.5\right)$ was added into $0.768 \mathrm{mg}$ Cy7.5 NHS ester in $76.8 \mu \mathrm{L}$ of DMF 
with stirring. The reaction was done at $4{ }^{\circ} \mathrm{C}$ for 5 hours under dark condition. The crude peptide conjugate was purified using a Shimadzu reverse phase high-performance liquid chromatography (RP-HPLC) (Kyoto, Japan) on a specific pre-set gradient (0 min: 95\% A \& $5 \%$ B, 25 min: $30 \%$ A \& 70\% B, 30 min: $5 \%$ A \& 95\% B, where A solvent is $0.1 \%$ TFA in pure water and B solvent is $0.1 \%$ TFA in acetonitrile), at $1 \mathrm{~mL} / \mathrm{min}$ flow rate, and with a UV detection at $280 \mathrm{~nm}$. The product solution was collected and lyophilized on a Savant SpeedVac concentrator. The purity and identity of Cy7.5-K-8AOC-BBN[7-14] $\mathrm{NH}_{2}$ was examined on a 4700 MALDI TOF/TOF mass spectrometer (Applied Biosystem Inc., now AB Sciex) at the University of Missouri Proteomics Center.

\section{Synthesis and purification of USPIO(Cy7.5)-BBN}

USPIO(Cy7.5)-BBN nanoparticle was derived through a four-step reaction. Step 1 and 2: surface modification to transfer from water insoluble to water soluble USPIO nanoparticle. Step 3: carboxylic acid functionalization of USPIO nanoparticle. Step 4: conjugation of Cy7.5-BBN to USPIO.

Synthesis of glucose-coated USPIO nanoparticle: Glucose (155 mg) in $5.6 \mathrm{~mL}$ DMF solution was preheated and mixed with oleic acid coated USPIO at a molar ratio of 25000:1 of glucose to the nanoparticle. The reaction was allowed for $1 \mathrm{hr}$ at $138^{\circ} \mathrm{C}$ on a Talboys standard dry block heater (Thorofare, NJ). A $5.8 \mathrm{~mL}$ brownish solution was obtained and cooled to room temperature. The mixture was then washed three times 
with ethanol and separated with a SuperMag separator (Ocean Nanotech, San Diego, CA). The obtained glucose coated USPIO was re-dispersed in Milli-Q water.

Synthesis and purification of casein-coated USPIO nanoparticle: Casein was pretreated with $0.01 \mathrm{M} \mathrm{NaOH}$ and lyophilized overnight on a Savant SpeedVac concentrator to obtain water soluble sodium caseinate powders. The glucose-coated USPIO solution was mixed with the sodium caseinate powder at a 78:1 molar ratio of casein to USPIO and was stirred at room temperature for 4 hrs. Freshly prepared $0.4 \%$ glutaraldehyde was then added into this solution (molar ratio of casein to glutaraldehyde at 2:1) dropwise to trigger a cross-linking reaction that was allowed for 1 hour at room temperature. A dark brown solution was obtained and proceeded for a purification in a $100 \mathrm{kDa}$ MWCO dialysis membrane to remove any small molecules that were not successfully cross-linked to the nanoparticles. The membrane dialysis was performed with gentle stirring in Milli-Q water on a shaker at $4{ }^{\circ} \mathrm{C}$ for three times and a total of 48 hours.

Surface amino group blockage reaction: The casein-coated USPIO nanoparticles have both amino groups $\left(-\mathrm{NH}_{2}\right)$ and carboxyl groups $(-\mathrm{COOH})$ on the surface. To create $\mathrm{COOH}$ functionalized USPIO nanoparticles, acetate NHS ester was added into the caseincoated USPIO solution to cap the amino groups on the casein, at a 60:1 molar ratio of acetate NHS ester to casein. The $\mathrm{pH}$ of the reaction mixture was adjusted to be 8.15 with $\mathrm{NaHCO}_{3}$, and the reaction was carried out at room temperature and $\mathrm{pH}$ of 8.15 for $1 \mathrm{hr}$. Finally, the solution was loaded into a $50 \mathrm{kDa}$ MWCO dialysis membrane (Spectrum 
Inc.) that was suspended in $500 \mathrm{~mL}$ Milli-Q water for another membrane dialysis purification for 48 hours.

Conjugation of Cy7.5-K-8AOC-BBN[7-14]NH $\mathrm{N}_{2}$ to USPIO: The final step is the conjugation reaction between the surface-modified hydrophilic USPIO-casein nanoparticle and Cy7.5-K-8AOC-BBN[7-14] $\mathrm{NH}_{2}$ to form USPIO(Cy7.5)-BBN nanoparticles. To approximately $0.893 \mathrm{mg}$ (in Fe) $\mathrm{COOH}$ functionalized USPIO solution, $2.298 \mathrm{mg}$ EDAC was added followed by the addition of $1.857 \mathrm{mg}$ Sulfo-NHS (molar ratio of EDAC : SulfoNHS : NH2(reactive group in Cy7.5-K-8AOC-BBN[7-14] $\mathrm{NH}_{2}$ ) = 46:33:1). The reaction mixture was stirred for 15 minutes and was added with $0.259 \mu \mathrm{mol}$ Cy7.5-K-8AOCBBN[7-14] $\mathrm{NH}_{2}$ (41.9:1 for BBN to the nanoparticle) in Milli-Q water. The conjugation reaction was allowed for 8 hours at $4{ }^{\circ} \mathrm{C}$ in the dark. The purification was again carried out by a $50 \mathrm{kDa}$ MWCO membrane dialysis for three times under dark and at $4{ }^{\circ} \mathrm{C}$.

\section{Determination of iron content of nanoparticles}

The iron concentration in USPIO nanoparticles was determined using Prussian blue staining spectrophotometric method. Standard solutions of different iron concentrations $(0.112,0.0896,0.0672,0.0448,0.0224,0 \mathrm{mg} / \mathrm{ml})$ were prepared with Ferridex (Bayer, Leverkusen, Germany). Each $200 \mu \mathrm{L}$ solution was added with $200 \mu \mathrm{L}$ 12.1 $\mathrm{N} \mathrm{HCl}$ for acid hydrolysis reaction at $80^{\circ} \mathrm{C}$ for 4 hours, followed by adding $400 \mu \mathrm{L}$ MilliQ water for dilution and $200 \mu \mathrm{L} 5 \%$ Prussian blue for staining. The absorbance of each solution was determined at $690 \mathrm{~nm}$ on a Shimazu 1601 UV-vis spectrophotometer 
(Kyoto, Kyoto Prefecture, Japan). A standard correlation of the light absorbance versus iron concentration was fitted to a linear curve according to the Beer's Law:

$$
\text { Absorbance }=\log \left(I_{0} / l\right)=\varepsilon \mathrm{LC}
$$

where $I_{0}$ and $I$ are the light intensity before and after passing the solution, $\varepsilon$ is the molar extinction coefficient (or molar absorptivity constant), $L$ is the path length of the sample cuvette, and $\mathrm{c}$ is the concentration of the solution. To prepare the sample solution, $20 \mu \mathrm{L}$ stock solution was diluted to $200 \mu \mathrm{L}$ with Milli-Q water and mixed with $200 \mu \mathrm{L} 12.1 \mathrm{~N} \mathrm{HCl}$ for $4 \mathrm{hrs}$ at $80^{\circ} \mathrm{C}$, followed by addition of $400 \mu \mathrm{L}$ water and $200 \mu \mathrm{L} 5 \%$ Prussian blue solution. The light absorption was also measured on the Shimazu 1601 UV-vis spectrophotometer. The iron concentration was estimated against the standard curve with triplicate measurements.

\section{Determination of peptide to nanoparticle ratio}

First, standard solutions of Cy7.5 in $150 \mu \mathrm{L}$ DMF at different concentrations $(0$, $6.25,12.5,25,50,100$ and $200 \mu \mathrm{M})$ were prepared in a 96-well Cellstar transparent microplate for the absorbance measurement on a Synergy H4 hybrid reader (Biotek, Winooski, VT) at $788 \mathrm{~nm}$ wavelength. The absorbance versus concentration was fitted to a linear curve based on the Beer's Law as well. The concentration of Cy7.5-BBN is estimated against the standard curve of Cy7.5 versus concentration and the molar ratio of 1:1 for BBN to Cy7.5. The iron content of USPIO(Cy7.5)-BBN was obtained by the Prussian blue staining method, and the molar concentration of the USPIO nanoparticles 
was estimated according to the information regarding the ratio of iron content to the molar concentration of nanoparticles given by the supplier. The peptide to USPIO ratio was then calculated using the molar concentration of Cy7.5-BBN to be divided by the molar concentration of iron oxide nanoparticle.

\section{Fourier transform infrared (FTIR) spectroscopy}

To substantiate that glucose and casein have been conjugated on the surface of USPIO, Fourier transform infrared (FTIR) spectroscopy was used to identify chemical bonds in the oleic acid coated USPIO, glucose coated USPIO and casein coated USPIO. FTIR spectra were acquired and analyzed on a Galaxy series 5000 FTIR spectrometer (Mattson ATI). Each spectrum in middle infrared range (4000 cm-1 to $400 \mathrm{~cm}-1)$ was an average of 16 scans, and the spectral resolution was set to be $2 \mathrm{~cm}^{-1}$. The FTIR samples were prepared using a small amount of dry sample (such as glucose, casein, oleic acid coated USPIO glucose coated USPIO, or casein coated USPIO) mixed with dry $\mathrm{KBr}$ powders. The mixture was pressed using a press for several tons in about $2 \mathrm{~min}$. The background of the spectrum was recorded using a pure $\mathrm{KBr}$ pellet.

\section{Transmission electron microscopy (TEM)}

The morphology and core sizes of casein coated USPIO and USPIO(Cy7.5)-BBN were studied on a JEOL 1400 transmission electron microscope with accelerating 
voltage of $40-120 \mathrm{kV}$. At the beginning of sample preparation, each nanoparticle solution was sonicated for 3 minutes, then $20 \mu \mathrm{L}$ was dripped on a carbon coated copper grid for a 5 minutes incubation. The specimen was air dried before being inserted into a specimen chamber of the TEM instrument.

\section{Dynamic light scattering (DLS)}

The hydrodynamic radius of glucose coated USPIO, casein coated USPIO, and USPIO(Cy7.5)-BBN in aqueous solution was measured using a dynamic light scattering instrument ALV/CGS-3 SLS/DLS system (ALV, Langen/Germany). The tracking of fluctuations of the light intensity, induced by the scattering effect of the nanoparticles' Brownian Motions in the light path, was lasted for 30 seconds in each run, followed by the analysis of the obtained data by a DLS autocorrelation function to come up with the hydrodynamic radius of the nanoparticles.

\section{MRI relaxivity determination}

The solutions of USPIO(Cy7.5)-BBN and Feridex were prepared in water. $\mathrm{R}_{1}$ and $R_{2}$ relaxation rates were determined at iron concentrations of $0.235,0.196,0.157$, $0.117,0.078$, and $0.039 \mathrm{mM}$. The measurements were repeated on two or more independently prepared samples to ensure consistency. A buffer matched blank sample $(0 \mathrm{mM})$ was also used in the relaxivity measurements of each sample. 
Measurements were performed using a 7 Tesla Bruker BioSpec AvancellI MRI system (Bruker BioSpin, Corporation) equipped with a volume radiofrequency (RF) coil (86 mm inner diameter) at $25^{\circ} \mathrm{C} . \mathrm{A} \mathrm{T}_{1}$-weighted MRI pulse sequence was applied with echo time $(T E)=15 \mathrm{~ms}$, repetition time $(T R)=500 \mathrm{~ms}$ and slice thickness $=1 \mathrm{~mm} . \mathrm{R}_{1}$ and $R_{2}$ were simultaneously measured using a RARE-T1+T2-Map pulse sequence with slice thickness $=1 \mathrm{~mm}$, matrix $=256 \times 128, \mathrm{FOV}=50 \times 30 \mathrm{~mm}, \mathrm{NEX}=1, \mathrm{TE}=11,22,33,44$, $55,66,77,88 \mathrm{~ms}$ and TR ranging from $0.482 \mathrm{~s}$ to $5 \mathrm{~s}$. Signal intensity of every sample was measured, and the relaxation rate was obtained by the inner fitting functions in the ParaVision 5.1 software (Bruker BioSpin Corporation, 2012). The final relaxivity value was derived from a linear fitting equation of the relaxation rates against the different concentrations of the nanoparticle samples through the Origin 8.5.0 (OriginLab Corporation, 2014).

\section{Cell culture and in vitro binding affinity}

PC-3 human prostate cancer cells were obtained from the American Type Culture Collection (Manassas, VA) and maintained by the Cell and Immunobiology Core Facility at the University of Missouri. PC-3 cells were grown in complete growth medium

[RPMI1640 media containing 10\% heat-inactivated fetal bovine serum (FBS), $1 \%$ penicillin from Invitrogen (Carlsbad, CA)] in a Forma water-jacketed incubator (Fisher Scientific) at $37{ }^{\circ} \mathrm{C}$ and $5 \% \mathrm{CO}_{2}$. Cells were grown for 3 days to approximately $90 \%$ 
confluence, detached with Trypsin (Invitrogen, Carlsbad, CA) in 0.25\% EDTA solution, washed and re-suspended in a fresh growth medium.

To determine the binding affinity (inhibitory concentration fifty percent: IC50), in vitro competitive cell binding assays were performed against gold standard ${ }^{125} \mid-\mathrm{Tyr}^{4}-\mathrm{BBN}$ for GRPrs. Briefly, $3 \times 10^{4}$ PC-3 human prostate cancer cells [RPMI1640 media containing $4.8 \mathrm{mg} / \mathrm{mL} \mathrm{HEPES}$, and $2 \mathrm{mg} / \mathrm{mL} \mathrm{BSA}(\mathrm{pH} 7.4)]$ were incubated at $37^{\circ} \mathrm{C}$ for $45 \mathrm{~min}$ in the presence of $30,000 \mathrm{cpm}{ }^{125} \mathrm{I}-\mathrm{Tyr}^{4}-\mathrm{BBN}$ and increasing concentration of USPIO(Cy7.5)BBN. The incubation period was followed by the aspiration of the reaction medium, and the cells were washed three times with ice-cold media. Cell-associated radioactivity was determined by counting in a Wizard 3" 1480 automatic gamma counter (PerkinElmer, Waltham, MA). This procedure was repeated for three times for a statistical purpose.

In vitro cellular microscopic imaging: uptake, blocking, internalization, Prussian blue staining

To determine the binding selectivity of USPIO(Cy7.5)-BBN to GRPr, in vitro cellular imaging was performed in PC-3 cells similar to previously described ${ }^{111}$. Briefly, for the uptake study, $1 \times 10^{6}$ PC-3 cells were treated with $0.142 \mathrm{nmol}$ USPIO(Cy7.5)-BBN (defined by $0.142 \mathrm{nmol}$ of BBN equivalent to $0.0067 \mathrm{nmol}$ of the nanoparticle) at $37^{\circ} \mathrm{C}$ $\left(5 \% \mathrm{CO}_{2}\right)$ for $50 \mathrm{~min}$. For the blocking study, $1 \times 10^{6} \mathrm{PC}-3$ cells were pre-incubated with $56.8 \mathrm{nmol}$ native BBN[1-14] for 10 min prior to $50 \mathrm{~min}$ incubation with USPIO(Cy7.5)$\mathrm{BBN}$ at $37^{\circ} \mathrm{C}$. The cells were then centrifuged $\left(1,000 \mathrm{~g} \mathrm{rcf}\right.$ at $4^{\circ} \mathrm{C}$ for $\left.3 \mathrm{~min}\right)$, the 
supernatant was removed, and the cells were washed one time with ice-cold medium and three times with ice-cold PBS, finally were re-suspended in 50ul PBS and covered with aluminum foil. In vitro internalization study was performed to assess the degree of USPIO(Cy7.5)-BBN internalized into PC-3 cells. Briefly, PC-3 cells were incubated with $0.142 \mathrm{nmol}$ USPIO(Cy7.5)-BBN at $37^{\circ} \mathrm{C}\left(5 \% \mathrm{CO}_{2}\right)$ for $50 \mathrm{~min}$, followed by an acidic wash with $\mathrm{pH}=2.5$ buffer $(0.2 \mathrm{M}$ acetic acid, $0.5 \mathrm{M} \mathrm{NaCl})$ for two times and an additional wash with cold PBS. All procedures were conducted in the dark. The cell-associated fluorescence signals were assessed using an Olympus IX70 (Shinjuku, Tokyo, Japan) inverted microscope equipped with bright field, phase and fluorescence optical filters at the MU molecular cytology core. A Cy7 filter set was used with the excitation at 673-748 $\mathrm{nm}$ and the emission at 765-835 $\mathrm{nm}$, and images were processed using Metamorph software. All cell samples were prepared in triplicates.

Prussian blue staining was performed to confirm the coexistence of USPIO and Cy7.5 in PC-3 cells. Briefly, $2 \times 10^{6}$ cells were incubated with $0.284 \mathrm{nmol}$ USPIO(Cy7.5)BBN for 4 hours at $37^{\circ}\left(5 \% \mathrm{CO}_{2}\right)$. The treated cells were centrifuged and washed three times with PBS to remove excessive USPIO, then fixed with $4 \%$ formalin for 30 minutes or longer, centrifuged again and washed with Milli-Q water. Cells were dropped onto glass slides and air dried, then covered with a solution of $1 \%$ potassium ferrocyanide (50\%/50\%) in $2 \% \mathrm{HCl}$ for $20 \sim 30$ minutes. The slides were rinsed thoroughly with Milli-Q water and covered with coverslips for microscopic imaging. 


\section{Animal model}

In vivo studies were performed in severely compromised immunodeficient (SCID) mice bearing human PC-3 prostate cancer xenografts. Animal studies were conducted in accordance with the highest standards of care as outlined in the NIH's guide for care and use of laboratory animals and in accordance with policy and procedures for animal research at the Harry S. Truman Memorial Veterans Hospital. Four-to-five-week-old SCID mice were obtained from Taconic (Germantown, NY). Mice were housed four animals per cage in sterile microisolator cages in a temperature- and humiditycontrolled room with a 12-hour light/dark schedule. The animals were fed with autoclaved rodent chow (Ralston Purina Company, St. Louis, MO) and provided with water ad libitum. Mice were inoculated with $7 \times 10^{6}$ PC-3 tumor cells in $0.1 \mathrm{ml}$ matri-gel on each right and left flank. Mice were used in NIRF imaging and MRI experiments between 3-4 weeks post inoculation of tumor cells. Average body weight of mice was 25-30 grams at the time of the study.

\section{In Vivo MRI}

MRI study of tumor-bearing animals was performed on 7 T Bruker AVANCE III BioSpec MRI scanner (Bruker BioSpin Inc., Billerica, MA) equipped with a gradient insert (400 mT/m, $115 \mathrm{~mm}$ I.D.) and a quadrature RF coil (35 mm ID). Mice were anesthetized with $1-2 \%$ isoflurane in oxygen via a nose cone over the entire imaging period. A respiratory sensor was placed on the abdomen for respiratory monitoring of vital signs 
using a Physiological Monitoring System (SA Instruments, Inc., Stony Brook, NY). Body temperature was maintained at $37^{\circ} \mathrm{C}$ with warm air circulating in the magnet bore. A rapid acquisition with relaxation enhancement (RARE) T1-weighted (T1W) imaging sequence was used to obtain coronal images, and a RARE-T2 sequence was applied to obtain axial images. For RARE-T1W sequence: TR / TE $=774 \mathrm{~ms} / 9 \mathrm{~ms}$, RARE factor $=4$, 16 slices, ST $=1 \mathrm{~mm}$ with no gap, matrix $=256 \times 256, \mathrm{FOV}=80 \times 40 \mathrm{~mm}$ (coronal), NEX $=$ 4. For RARE-T2 sequence, $T R=2.5 \mathrm{~s}, \mathrm{TE}=11,22,33,44,55,66,77,88 \mathrm{~ms}$, RARE factor $=$ 4, 16 slices, $\mathrm{ST}=1 \mathrm{~mm}$ with no gap, matrix $=128 \times 128, \mathrm{FOV}=30 \times 30 \mathrm{~mm}$ (axial), $\mathrm{NEX}=$ 4. After the baseline scan had been completed, $87 \mu \mathrm{g}$ (Fe) of USPIO(Cy7.5)-BBN in $150 \mu \mathrm{l}$ isotonic saline was injected as a bolus via tail vein and CE-MRI images were obtained at 4, 24 and $48 \mathrm{~h}$ after injection.

Image analysis and processing were performed using ParaVision 5.1 software (Bruker BioSpin Corporation, 2012). Regions of interest (ROIs) were manually drawn on the tumor tissue, muscle near the tumor, kidney cortex, and liver for each time point. Signal intensity (SI) was measured as the mean of the intensity over the segmented ROI. The contrast enhancement ratio (CER) for an ROI was calculated according to the equation:

$$
\mathrm{CER}=\left(\mathrm{CNR}_{\text {post }}-\mathrm{CNR}_{\text {pre }}\right) / \mathrm{CNR} \text { pre } \times 100 \%
$$

Where CNR is the contrast to noise ratio: $\mathrm{CNR}=\left(\mathrm{SI}_{\text {tumor }}-\mathrm{SI}_{\text {muscle }}\right) / \boldsymbol{\sigma}_{\text {noise. }}$ 


\section{NIRF molecular imaging}

In vivo and ex vivo NIRF imaging were performed on an IVIS Spectrum imaging system (PerkinElmer, Waltham, MA) equipped with a cooled charge-coupled device (CCD) camera and a 150-W quartz halogen light source. The NIRF images were acquired at a filter setting (excitation $745 \mathrm{~nm}$; emission $820 \mathrm{~nm}$ ) with the parameters: exposure time (2 sec), f/stop (4), binning (M)8, and field of view $(13.2 \mathrm{~cm})$. Fluorescence semiquantification was performed using Living Image 4.4 (Xenogen). Region-of-interests (ROIs) were drawn on the tumors and background tissues in the NIRF images and the expression of fluorescence emission intensity was normalized to average radiant efficiency $\left([\mathrm{p} / \mathrm{sec} / \mathrm{cm} 2 / \mathrm{sr}] /\left(\mu \mathrm{W} / \mathrm{cm}^{2}\right)\right)$.

After the baseline scan had been completed, mice were given $87 \mu \mathrm{g}$ (Fe) of USPIO(Cy7.5)-BBN in $150 \mu \mathrm{l}$ isotonic saline via tail vein intravenous injection (i.v.) and were imaged at $0.5 \mathrm{hr}, 1 \mathrm{hr}, 24 \mathrm{hr}, 48 \mathrm{hr}$. For the blocking study, mice were given i.v. coinjection of a native BBN [1-14] solution $(50 \mu \mathrm{g} / 50 \mu \mathrm{l})$ and $87 \mu \mathrm{g}(\mathrm{Fe})$ USPIO(Cy7.5)-BBN, and were imaged at $0.5 \mathrm{hr}, 1 \mathrm{hr}, 24 \mathrm{hr}$ and $48 \mathrm{hr}$ post injection. After the time point at 48 hours post injection, mice were sacrificed by cervical dislocation under anesthesia (4\% isofluorane in $1 \mathrm{~L} / \min \mathrm{O}_{2}$ ). Organs were collected, rinsed with PBS and subjected to the following ex vivo NIRF imaging. 


\section{Histopathology}

Liver, kidneys, and tumors of the mice from the in vivo study were fixed in $10 \%$ neutral-buffered formalin and embedded in paraffin blocks, and slices of $5 \mathrm{~mm}$ thickness were made on a microtome. Tissues were stained with Prussian blue (potassium ferrocyanide $10 \% / \mathrm{HCl} 20 \%(\mathrm{v} / \mathrm{v})$ ) and counterstained with hematoxylin and eosin (H\&E) (or nuclear fast red for tumor specimens). The images were taken using a Leica DM5500B microscope (Wetzlar, Germany).

\section{Statistical analysis}

Quantitative data were expressed as the mean \pm SD. Means were compared by analysis of a student's $t$-test. $P$ values of less than 0.05 were considered to be statistically significant.

\section{Results}

\section{Synthesis and Purifications}

USPIO-casein was generated via a cross-linking reaction of casein on the oleic acid coated USPIO. The casein coating was confirmed to be present on the surface of the USPIO through FTIR. As shown in Figure 3.3, iron oxide stretching bond was found in the spectrum of glucose coated SPIO at $580 \mathrm{~cm}^{-1}$. The spectrum of casein displays both 
C-N stretching bond at $1533 \mathrm{~cm}^{-1}$ and $\mathrm{N}-\mathrm{H}$ bending vibration bond at $1455 \mathrm{~cm}^{-1}$ as the characteristic peaks of peptide bonds. While the spectrum of USPIO-casein displays all the three peaks, and therefore, verifying the formation of the casein coating on the USPIO.

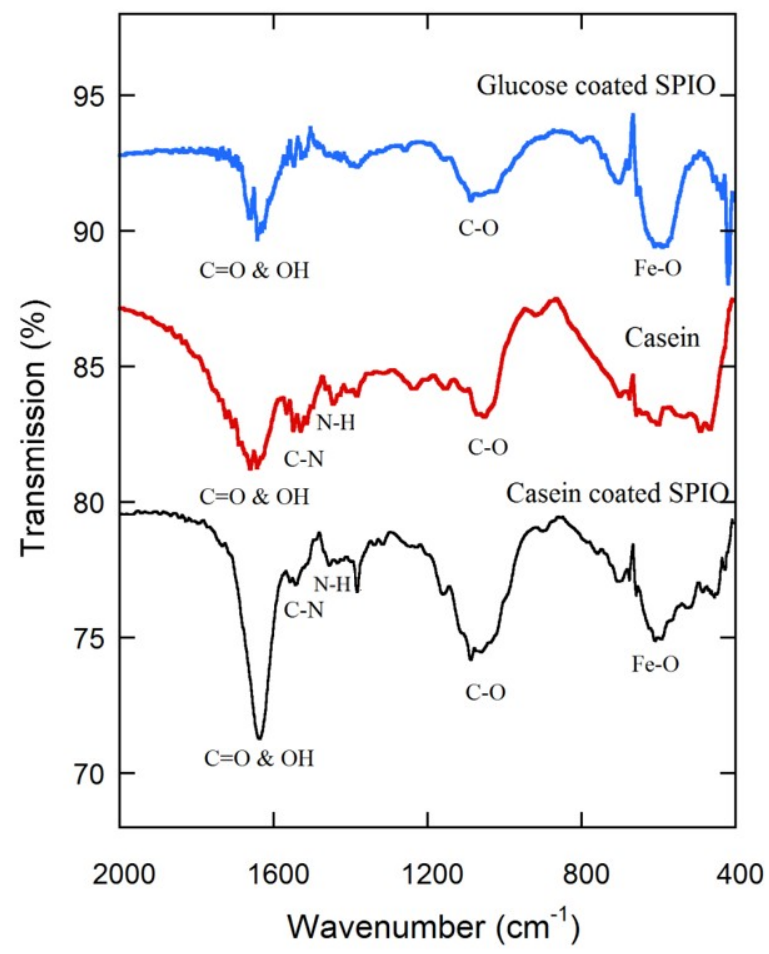

Figure 3.3 FTIR spectra of glucose coated USPIO nanoparticles (blue), pure casein (red) and casein coated USPIO nanoparticles (black).

On the other hand, the product of the conjugation reaction between the agonist BBN and Cyanine 7.5 was purified by HPLC and was measured to have a purity over 95\% (Figure 3.4). The solution of the third peak was collected and was validated to be agonist BBN-Cy7.5 by the mass spectrum result (Figure 3.5). In the result, the measured value 
was 1840.004, which was consistent with the theoretical molecular weight of our desirable compound, 1840.056 .

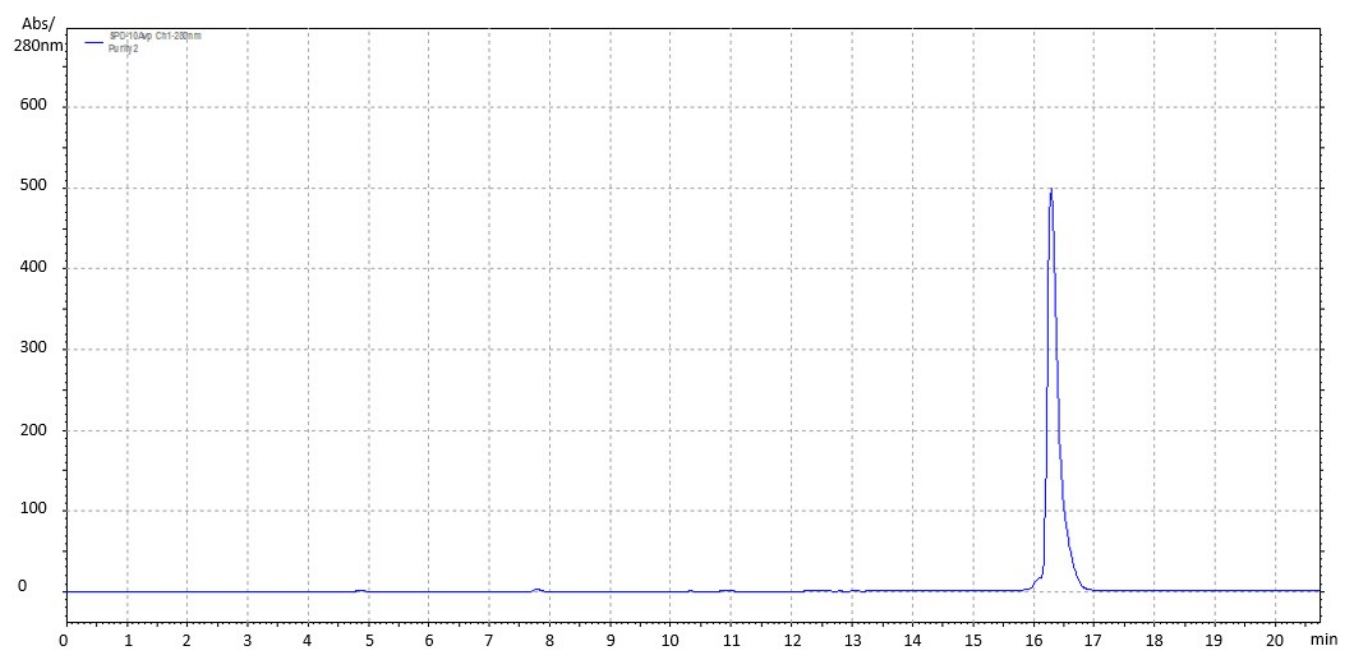

Figure 3.4 HPLC profile of the BBN-Cy7.5 with a purity over $95 \%$.

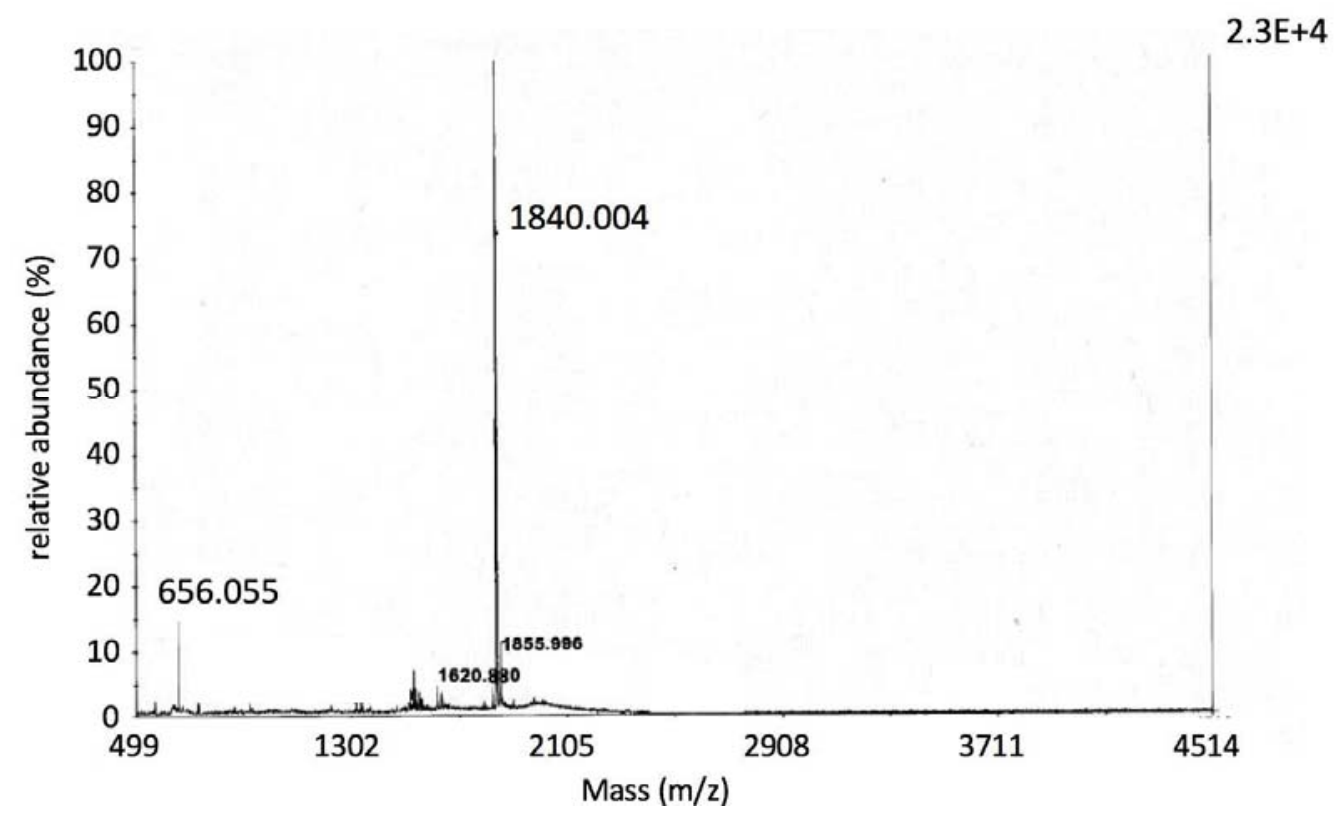

Figure 3.5 Mass spectrum of the BBN-Cy7.5 or K(Cy7.5)-8AOC-BBN[7-14]NH2. The MW in the figure is $1840.0 \mathrm{Da}$, matching the theoretic MW, 1840.1Da. 


\section{Core size and hydrodynamic size determination}

The core sizes of the nanoparticles, as shown in the TEM images (Figure 3.6a, b, c), were unchanged at all stages $(4.77 \pm 0.43 \mathrm{~nm}$ for the glucose-USPIO, $4.79 \pm 0.38 \mathrm{~nm}$ for the casein-USPIO and $4.93 \pm 0.31 \mathrm{~nm}$ for the USPIO(Cy7.5)-BBN (Figure 3.6d)). The hydrodynamic diameter by number weighting (Figure 3.7) for the USPIO(Cy7.5)-BBN was determined to be $37.3 \pm 13.8 \mathrm{~nm}$.
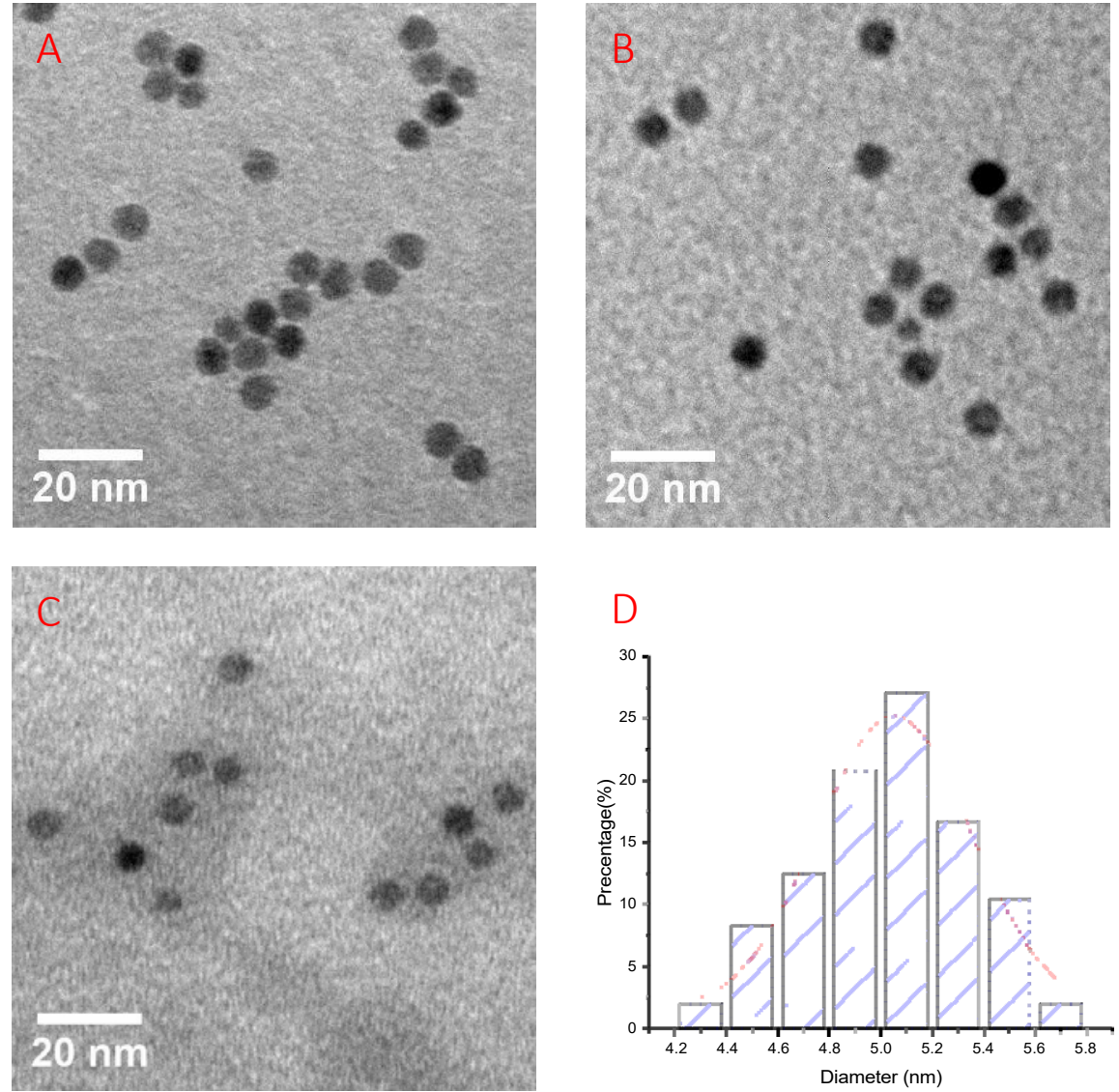

Figure 3.6 TEM of (A) glucose treated USPIO (step 1), (B) casein coated USPIO (step 2), (C) USPIO(Cy7.5)-BBN (step 3). (D) Histogram of the core diameter of USPIO(Cy7.5)-BBN. 


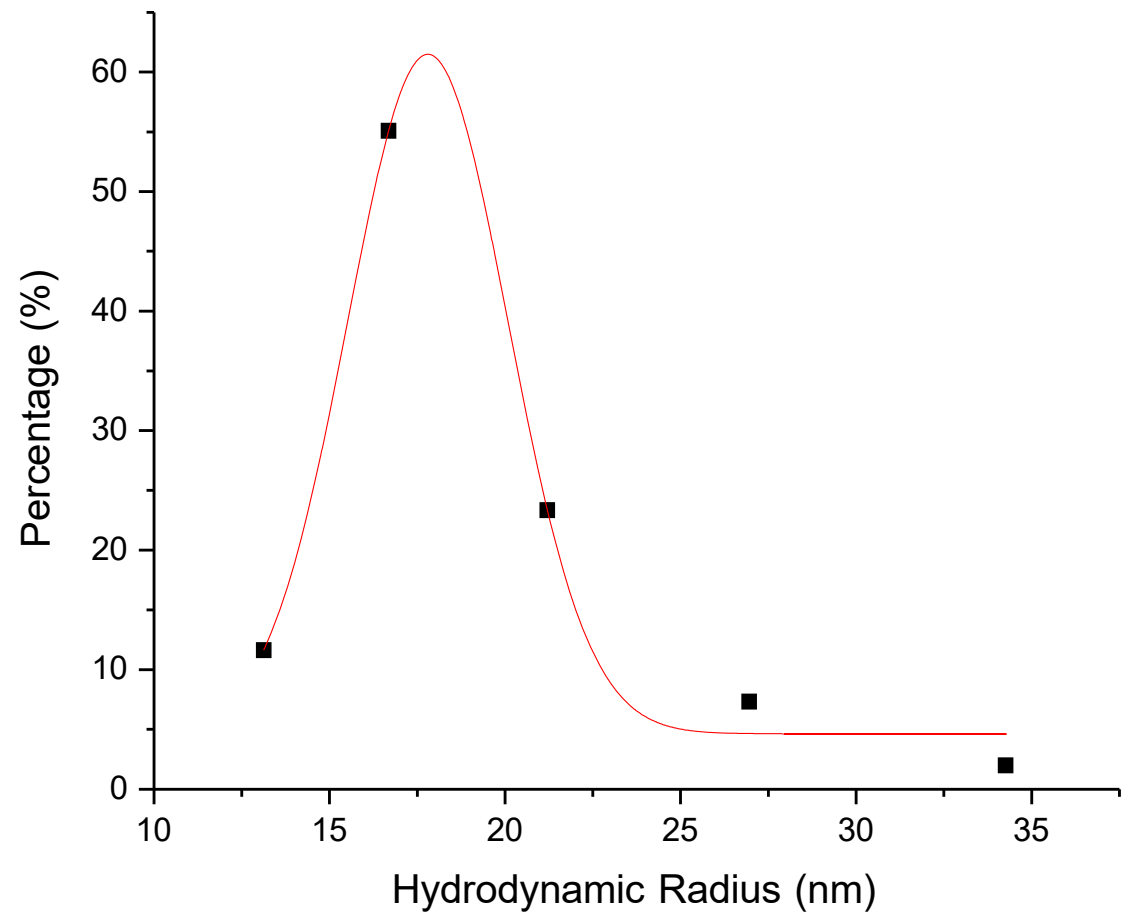

Figure 3.7 Number weighted hydrodynamic radius of USPIO(Cy7.5)-BBN.

\section{MRI Relaxivity Measurements of USPIO(Cy7.5)-BBN}

A series of sample solutions (Figure 3.8a) with different iron contents were placed into a Bruker 7T MRI scanner for measuring relaxivity that determines the MRI contrast enhancement ability of the contrast agent. The $r 2$ relaxivity of the USPIO(Cy7.5)-BBN was measured to be $70.2 \mathrm{~s}^{-1} \mathrm{mM}^{-1}$ against the fitting curve with different concentrations of the compound (Figure 3.8b), based on the equation $R_{2}=$ $\left(R_{2}\right)_{0}+C^{*} r 2$, where $C$ is the concentration and $R$ is the relaxation rate, $\left(R_{2}=1 / T_{2}\right)$. The 
outcome of this study is positive when comparing to other similar contrast agents at the same core size under developments.

A

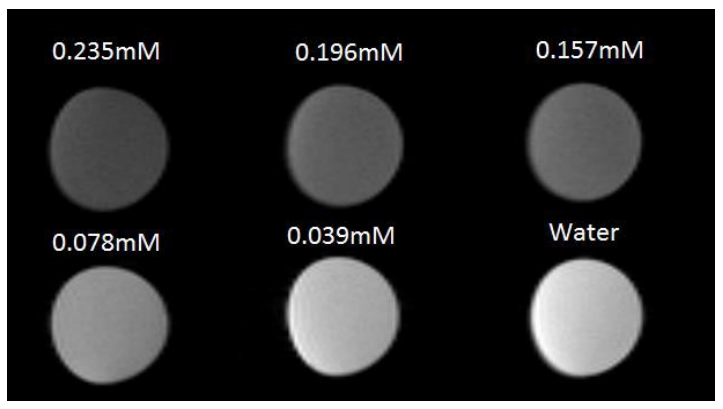

B

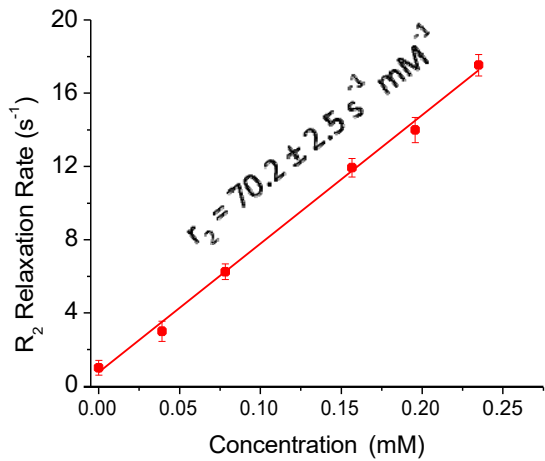

Figure 3.8(A) T2-weighted MRI image at TR of $2500 \mathrm{~ms}$ and TE of $55 \mathrm{~ms}$ for solutions with different concentrations of USPIO(Cy7.5)-BBN. (B) Linear fitting of relaxation rate at different concentration.

\section{Binding Affinity of USPIO(Cy7.5)-BBN to Prostate Cancer Cells}

The in vitro results (Figure 3.9A, B, C) revealed that the USPIO(Cy7.5)-BBN is able to bind to prostate cancer (PC-3) cells with a high selectivity and would be significantly inhibited in the presence of unlabeled BBN [1-14]. The internalization study showed most of the USPIO(Cy7.5)-BBN remained inside PC-3 cells after acidic wash, validating the internalization of this compound for PC-3 cells. In the cellular experiment with Prussian blue staining, internalized iron oxide nanoparticle would be transferred to be iron ions in an acidic environment and further be stained by potassium ferrocyanide. As inject 3.9D, E display, coexistence of iron elements with Cy7.5 in PC-3 cells was confirmed, where the red represented fluorescence of Cy7.5 and the dark blue 
represented the Prussian blue from iron elements. The binding affinity of the USPIO(Cy7.5)-BBN was quantitatively evaluated by ${ }^{125} \mathrm{I}-\mathrm{Tyr}^{4}-\mathrm{BBN}$ competitive binding assay. In result, the IC50 value (Figure 3.10 ) was figured out to be $2.5 \pm 0.7 \mathrm{nM}$ for the USPIO(Cy7.5)-BBN, indicating a high bind affinity to GRPr of PC-3 cells.
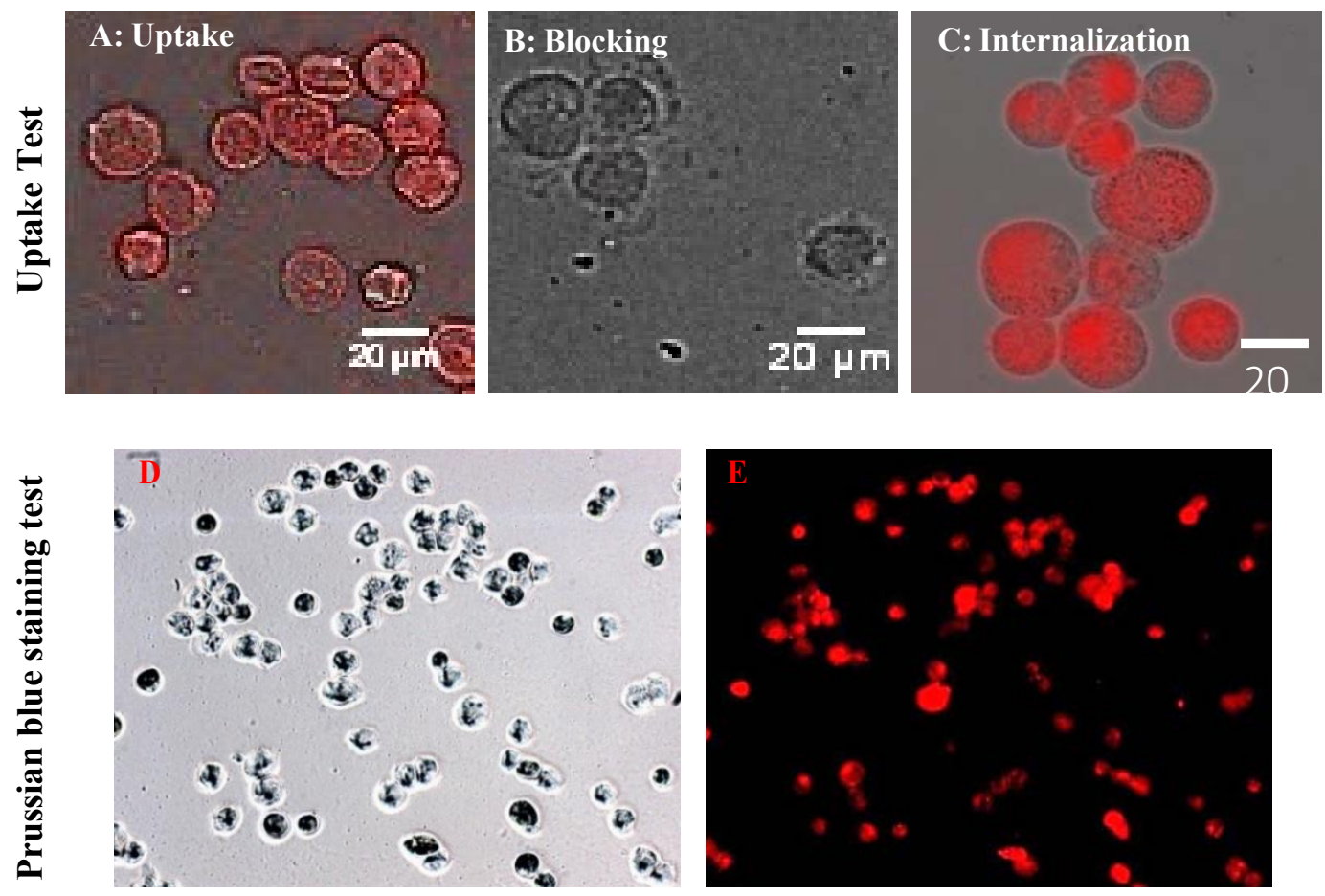

Figure 3.9 Microscopic cellular images. (A) Uptake: PC-3 cells treated with USPIO(Cy7.5)BBN. (B) Blocking: PC-3 cells pre-treated with 400-fold BBN[1-14], followed by the incubation with USPIO(Cy7.5)-BBN. (C) Internalization: PC-3 cells treated with USPIO(Cy7.5)-BBN, and further washed with $\mathrm{pH}=2.5$ buffer. (D) Prussian blue staining test of USPIO(Cy7.5)-BBN (dark blue) bound to PC-3 cells in a bright field image. (E) Prussian blue staining test of USPIO(Cy7.5)-BBN (dark blue) bound to PC-3 cells in a fluorescence image (red represents Cy7.5). 


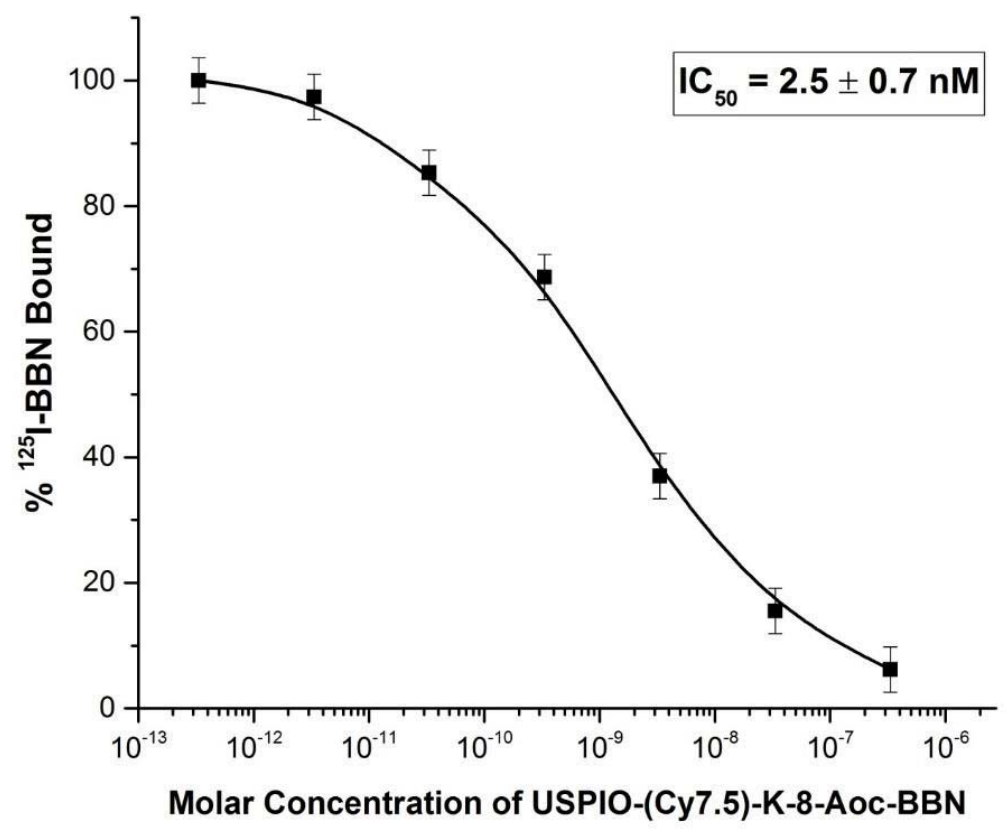

Figure 3.10 In vitro binding affinity of USPIO(Cy7.5)-BBN in PC-3 cells. IC50 $=2.5 \pm 0.7 \mathrm{nM}$, by $125 \mathrm{I}-\mathrm{Ty}$ r4-BBN competitive binding assay.

\section{In Vivo NIRF \& MRI Molecular Imaging}

The specific binding was evaluated in a PC-3 xenograft model on NIRF imaging and $\mathrm{MRI}$ respectively. All mice were assigned into the uptake group and the blocking group, where two mice in the uptake group were intravenously injected with $87 \mu \mathrm{g}$ (Fe)

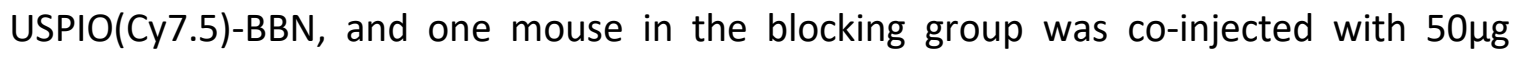

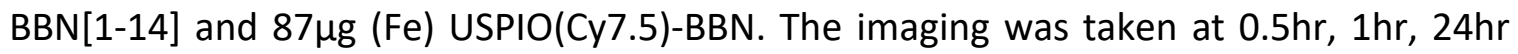
and $48 \mathrm{hr}$ post injection. Qualitatively, tumor's signal is stronger in uptake group than blocking group, especially at $48 \mathrm{hr}$ post injection (Figure 3.11a). In parallel to the NIRF study, the MRI study was taken at $4 \mathrm{hr}, 24 \mathrm{hr}$ and $48 \mathrm{hr}$ post injection (Figure $3.12 \mathrm{a}$ ), in 
which the contrast of tumors to muscle was significantly increased after the injection of the USPIO(CY7.5)-BBN. The contrast enhancement ratio (CER) was calculated based on these MRI images with the equation CER $=\left[(C N R)_{\text {pre }}-(C N R)_{\text {post }}\right] /(C N R)_{\text {pre }} \times 100 \%$ where CNR $=\left(I_{\text {tumor }}-I_{\text {muscle }}\right) / \sigma_{\text {noise }}$ (Figure $\left.3.12 \mathrm{~b}\right)$. The CER of the uptake group, especially at $4 \mathrm{hr}$ and $24 \mathrm{hr}$ p.i., was significantly higher than the CER of the blocking group. At the end of

this study (48hr p.i.), mice in the two groups were sacrificed, and various organs, including heart, lungs, liver, spleen, kidneys, pancreas, bladder, tumor, muscle and tibia were collected for the ex vivo NIRF study (Figure 3.11b). The result showed that the tumor to muscle ratio was equal to 4.8 , which is high than that of 2.9 for the blocking group. One thing worth noting that pancreas, which is the only normal organ rich of GRP receptors, had stronger fluorescent signal intensity in the uptake group than that in the blocking group. Besides that, an interesting phenomenon was observed for the result that the signal intensity of mice's liver in the blocking group was obviously higher than that in the uptake group, but in contrast to that, the kidneys' signal intensity was lower in the uptake group than that in the blocking group. 

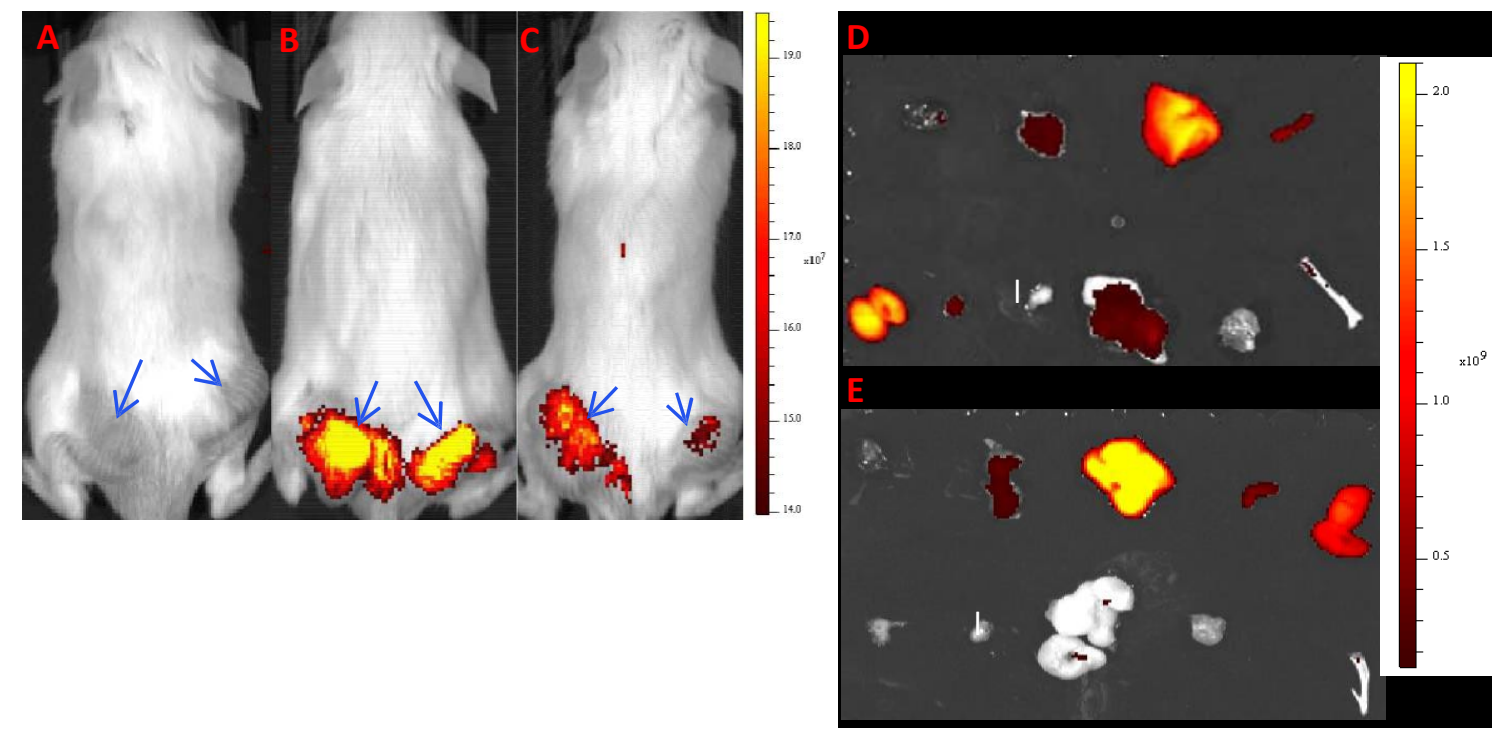

Figure 3.11 IVIS imaging of tail vein injection of USPIO(Cy7.5)-BBN in SCID mice bearing PC-3 tumors for pre-injection(A), the uptake group at $48 \mathrm{hr}$ post injections(B) and the blocking group at $48 \mathrm{hr}$ post injection(C). The left is the ex vivo NIRF imaging of organs collected $48 \mathrm{~h}$ post injection for the uptake group(D) and the blocking group(E). Number denotes: $\mathrm{H}$, heart; Lu, lung; Li, liver; Sp, spleen; Ki, kidney; Pa, pancreas; BI, bladder; Tu, tumor; Mu, muscle; Ti, tibia. 
A
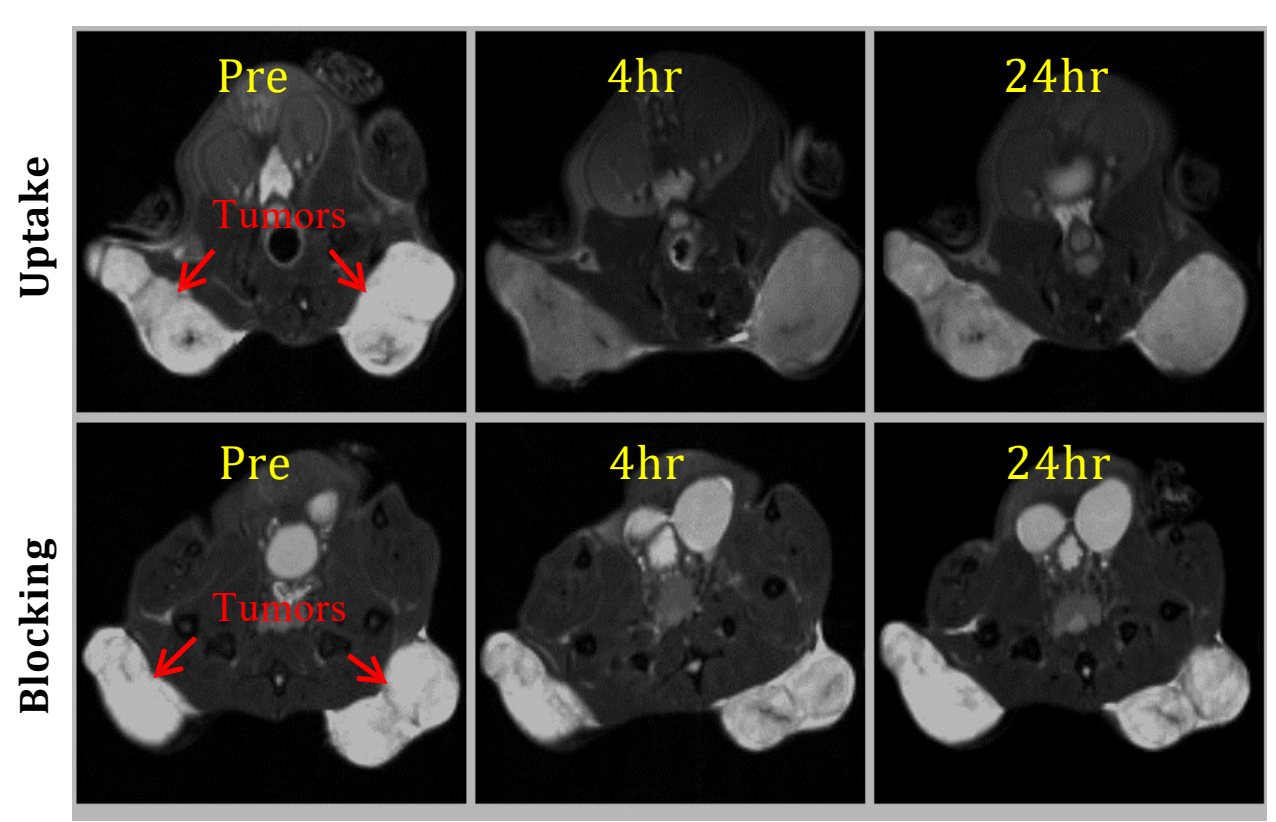

B

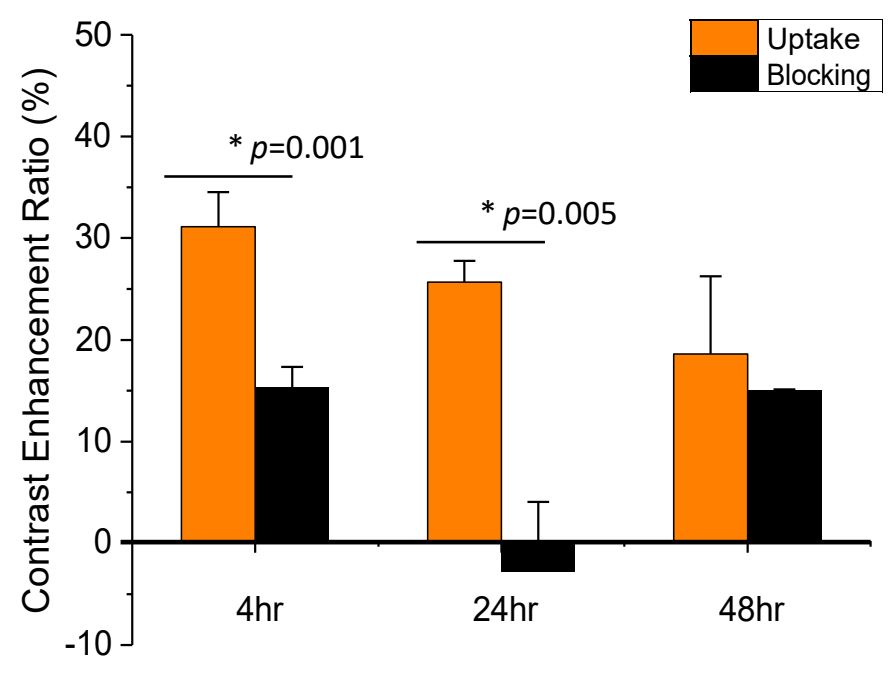

Post Injection Time

Figure 3.12(A) Representative T2-weighted MRI of uptake group (upper panel) compared to the blocking group (lower panel) pre and post i.v. injections of USPIO(Cy7.5)-BBN. TR $=2347 \mathrm{~ms}$ and TE $=33 \mathrm{~ms}$. (B) The contrast enhancement ratio (CER) of uptake and blocking groups at $4 \mathrm{hr}, 24 \mathrm{hr}$ and $48 \mathrm{hr}$ p.i.. 


\section{Histopathology}

The distribution patterns of iron oxide nanoparticle in the tumors, livers, and kidneys were explored in the two groups. All the slices of livers and kidneys were dualstained with Prussian blue and H\&E, while tumor specimens were dual-stained with Prussian blue and nuclear fast red. As Figure 3.13A and Figure 3.13E display, the captured USPIO was distributed unevenly in kidneys. Regions with concentrated USPIO can be observed in the kidneys from the uptake group, but rarely in the kidneys from the blocking group, implying the lower portions of USPIO removed by the renal excretion in the blocking group, which is consistent with the result of ex vivo NIRF study. As shown in Figure 3.13B, regions with dense stained USPIO were hardly observed in the liver. Since the ex vivo NIRF result shows that most of USPIO nanoparticles were excreted through liver, it indicates that the USPIO captured by hepatic macrophages were digested into small particles or iron elements that are almost invisible. As Figure 3.13C and Figure 3.13D show, the distribution of USPIO is not uniform in tumors, implying heterogeneity in the USPIO's access to the tumor cells in the solid tumor. Blue spots can be observed in the tumors from the uptake group, but not much in the blocking group (Figure 3.13F), suggesting a selective delivery of the USPIO to the prostate tumor. By acknowledging the contrast of iron deposit in tumors between the two groups is not striking at this time point (48 hours p.i.), as well as the low CER difference between the two groups in the in vivo MRI at this time point (Figure 3.12B), a better histopathological outcome is expectable if the sampling time is earlier. 

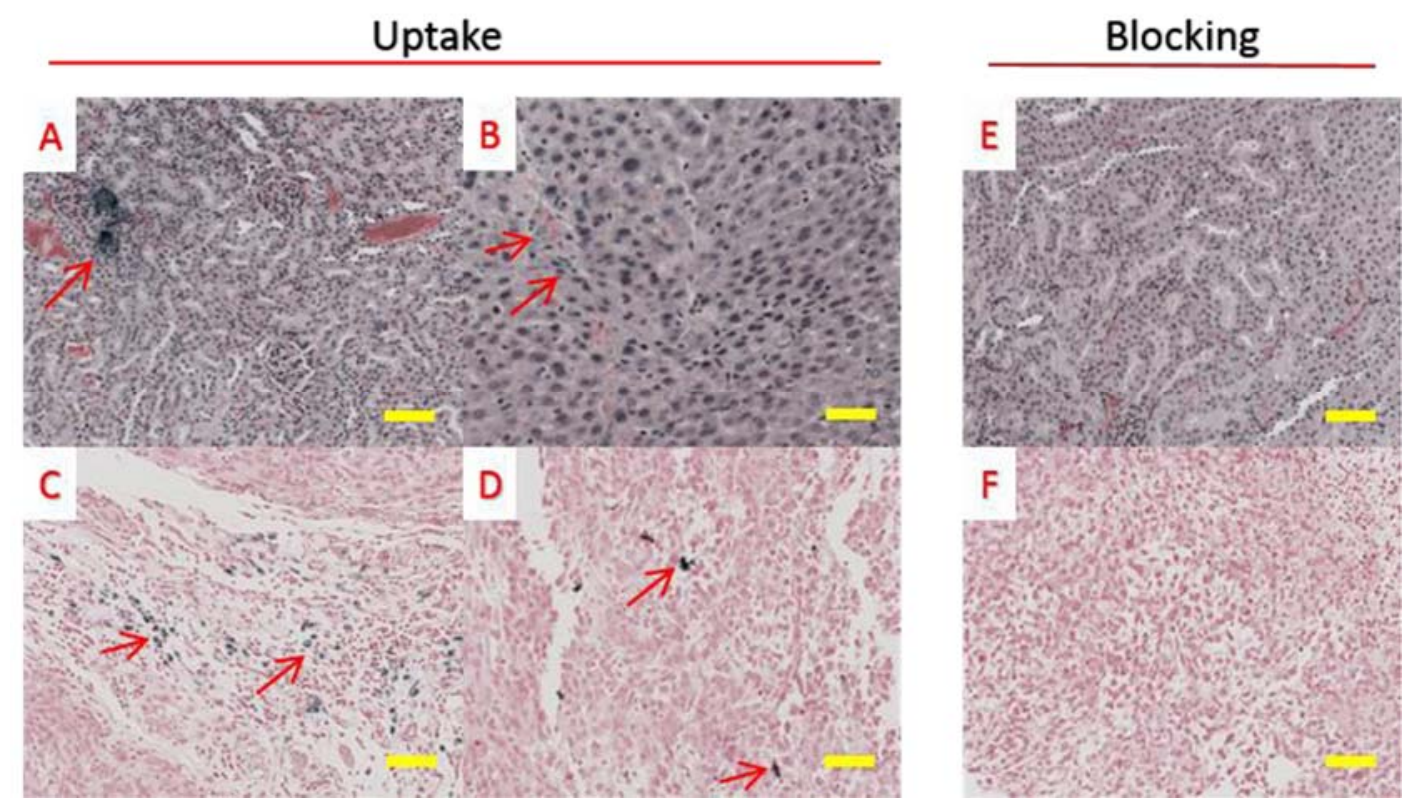

Figure 3.13 Histopathological results: (A) Kidney in the uptake group. (B) Liver in the uptake group. (C, D) Tumors in the uptake group. (E) Kidney in the blocking group. (F) Tumor in the blocking group. Arrows indicate the USPIO nanoparticles with Prussian blue staining. The scale bar represents $100 \mathrm{um}$.

\section{Discussion}

Near-infrared fluorescence (NIRF) imaging with a high sensitivity and a multidetection capability, could be potentially applied in intra-operative procedures and imaging guided therapies. ${ }^{56,82,83}$ The NIRF imaging relies on the use of NIRF probes that emit optical signals in the near infrared spectral region (600-900nm), bringing about a desirable penetration depth into human body, since water, lipid and hemoglobin display the minimum absorption and scattering in this wavelength range. ${ }^{84-86}$ Although NIRF imaging features high sensitivity, its use is limited by the bad display of anatomical details, while this defect could be perfectly complemented by the characteristics of MRI. 
Thus, the combination of MRI and NIRF is a promising candidate for noninvasive multimodal imaging procedures that output images with high spatial resolutions, sensitivity and rich anatomical details. ${ }^{87}$

The delivery of USPIO to tumor neo-vasculatures and the prostate tumor is attributed to both binding effect of the targeting vector to the corresponding receptors and a passive delivery by the enhanced permeability and retention (EPR) effect. ${ }^{18,19}$ Long-circulation time is the prerequisite for the drug to remarkably accumulate in tumor site, and the factors including coating materials and sizes can profoundly affect circulation time of nanoparticles. SPIO with a hydrodynamic diameter over $200 \mathrm{~nm}$ will be rapidly removed by the macrophages of liver and spleen, while SPIO with a hydrodynamic diameter less than $10 \mathrm{~nm}$ will be rapidly eliminated via the renal system. ${ }^{64,88,89}$ Therefore, the SPIO ranging from $10 \mathrm{~nm}$ to $100 \mathrm{~nm}$ is particularly fit for prostate cancer targeting. About the coating materials, since conventional iron oxide nanoparticle made from the co-precipitation methods or pyrolysis methods is hydrophobic, various strategies have been made to improve the hydrophilicity of SPIO ${ }^{90-}$ 92 that lead to an elevation of circulation time.

In this work the ultra-small iron oxide nanoparticle $(5 \mathrm{~nm})$ with oleic acid coating was encapsulated with casein matrix, followed by the conjugation reaction with the fluorescence dye, Cy7.5, and the peptide, agonist bombesin. Although the small core size of the nanoparticle likely leads to a low relaxivity as a tradeoff of elongated circulation time, the $\mathrm{r} 2$ relaxivity value of the USPIO(Cy7.5)-BBN was measured to be 
$70.2 \mathrm{~s}^{-1} \mathrm{mM}^{-1}$ at 7 Tesla, which is still a positive value at this core size. Cy7.5, the fluorescent dye selected to conjugate with the USPIO, was expected to have a relatively high depth of penetration due to its long emission wavelength (maximum emission at $808 \mathrm{~nm}$ ) based on the principle that the penetrability of light increases with its wavelength. The USPIO(Cy7.5)-BBN was confirmed to target to prostate cancer with a high binding affinity and specificity by the in vitro and the in vivo studies. For the in vitro study, the phenomenon was observed that the USPIO(Cy7.5)-BBN was fully competed by 400 -fold BBN [1-14] during the incubation with PC-3 cells in the blocking study, validating the specific binding of USPIO(Cy7.5)-BBN to PC-3 cells. Moreover, the IC50 value was determined to be $2.5 \pm 0.7 \mathrm{nM}$ (in nanoparticle), indicating a high binding affinity to PC-3 cells. The USPIO(Cy7.5)-BBN was demonstrated to be internalized in PC-3 cells in the internalization study, which was in agreement with the nature of the agonist BBN (K-8AOC-QWAVGHLM-NH2). About the in vivo study, in view of the much faster blood circulation of the BBN derivative as a small molecule than the BBN conjugated nanoparticle, the BBN[1-14] and the USPIO(Cy7.5)-BBN were co-injected into the PC-3 xenograft model where the BBN[1-14] was assumed to approach tumor sites faster than the BBN conjugated nanoparticles, for the following NIRF and MRI detections. In the NIRF study, the tumors of mice in the uptake group displayed a significantly higher fluorescent signal intensity than that in the blocking group. Since the direct measurement of fluorescent signal from the surface of mice is not accurate to quantitate the fluorescence, the animals were sacrificed and dissected for an ex vivo study, where the measurement of tumor to muscle's fluorescent strength ratio also 
demonstrated a higher uptake of the UPSIO(Cy7.5)-BBN in the prostate tumors from the uptake group than that from the blocking group.

One interesting phenomenon worth noting is that the signal contrast between liver and kidney was significantly altered when BBN[1-14] was co-injected with the nanoparticles. The signal intensity of the mouse's liver in the blocking group was obviously higher than that in the uptake group, but in contrast to that, the kidneys' signal intensity was lower in the uptake group than that in the blocking group. This phenomenon infers a change of excretion with the co-injection of BBN[1-14] and the reason is probably the antidiuretic effect of $\mathrm{BBN}$ reported in a previous research paper. ${ }^{93}$

In addition to the in vivo NIRF studies, the in vivo MRI studies were performed simultaneously. The T2-weighted MRI images of the two groups revealed the changes of the tumors' signal intensity in the two groups that the darkening of the tumor's signal in the uptake group is visible, but this change is unclear in the blocking group. The contrast enhancement ratio (CER) was calculated on these MRI T2 weighted images. The higher CER of tumors in uptake group at $4 \mathrm{hr}$ p.i. and $24 \mathrm{hr}$ p.i. indicates the significant enhancement of contrast between tumors and muscles within this period of time. While the disappearance of the CER difference between the two groups at $48 \mathrm{hr}$ p.i. implys the excretion or biotransformation of the USPIO(Cy7.5)-BBN from prostate cancer had been mostly done in the uptake group at this time point. 
According to the histopathological result, a small amount of the compound could be found inside the dense cancer cell clusters in the uptake group, but rarely in the blocking group. As it shows in MRI study, contrast enhancement ratio of the two groups at $48 \mathrm{hr}$ p.i. were very close, a striking difference in the quantity of iron deposit in cancer cell clusters between the uptake group and the blocking group is expectable if sampling time is earlier.

Up to now, long term toxicity of iron oxide nanoparticle has not been documented. ${ }^{94,95}$ The administrated USPIO is often found largely existing in liver, spleen and bone marrow, and it is rational because macrophages are in a significant amount in these organs. Iron oxide nanoparticle has a strong tendency to be captured by these macrophages from blood circulation and further be decomposed into iron elements in endosomes and lysosomes where acidic environment presents. ${ }^{96}$ Finally, these free iron elements will be merged to cellular iron pool and be utilized for the generation of hemoglobin. In spite of this, there is an adverse effect found that when cells are over exposed to USPIO, the formation of excess reactive oxygen species (ROS) may occur and may result in apoptosis or cell death by influencing cellular normal functions..$^{89,96}$ Therefore, the reduction of dosage fulfilled by the specifically targeted delivery of SPIO is very meaningful that too many negative physiological responses can be prevented. During our in vivo studies, the mice with administrations behaved very normally, indicating no severe toxicity for this compound. 
The casein, as the major component of bovine milk protein, is a degradable material that can help the USPIO to avoid large immunoreactions when encapsulating it. Although the stability of the USPIO-casein is seemingly very excellent and no obvious precipitation was found at bottom of the container in three months, the dissociation of casein matrix from the USPIO core is still possible in bio environment. This worry was eliminated as the phenomenon observed that the significant hypointensity in tumor site was found in MRI T2 weighted image, concurrent with the elevation of signal intensity at the same position in NIRF image. This outcome confirms the co-existence of Cy7.5, USPIO and casein in tumor site, and proves the integrity of this drug in the process of delivery.

The USPIO(Cy7.5)-BBN can be extended to therapeutic applications with a loading of therapeutic agents. With the combined MRI and NIRF imaging modalities, therapy is able to be real-time monitored and evaluated. Besides that, the biodistribution and pharmacokinetics of the compound are traceable when the platform is loaded with radiolabeled agents and is detected through PET.

In conclusion, the USPIO(Cy7.5)-BBN was verified to significantly enhance the contrast of prostate cancer in NIRF system and MRI, via the specific binding affinity of bombesin to GRPR receptors. In the future work, an agent with triple modality (MRI/NIRF/PET) and formulation with therapeutic efficacy could be designed and tested for highly accurate, sensitive detection and personalized therapy. 


\section{Acknowledgement}

I appreciate Dr. Zongrun Jiang's help and guidance in HPLC operation.

I would like to thank Fengfei Wang's and Dr. Miao Zhang's help in FTIR and DLS sample preparation and measurements, Dr. Bandari, Rajendra P's help in IC50 measurements, Lisa Watkinson and Terry Carmack's help in SCID mice's inoculation, MU Electron Microscopy (EM) Core Facility's support in TEM practice, MU Molecular Cytology Core's support in fluorescent microscope practice. 


\section{Chapter IV. Cancer Cell Specific Silica-coated Iron Oxide Nanoparticles for Photoacoustic Detection of Prostate Cancer}

\section{Introduction}

According to the cancer statistics from American Cancer Society, 90\% of human cancer deaths are caused by metastasis of cancer cells. Circulating cancer cells (CTCs) are thought to be the origin of metastasis. CTCs are those cells shed from the primary tumor, traveling through blood and lymphatic system to form secondary tumors in distant sites. As a sign of cancer cells' generation and metastasis, the detection of CTCs is significant for early-stage cancer diagnosis, cancer prognosis and monitoring therapeutic response of cancer patients. ${ }^{129-131}$ However, the detection of the rare CTCs in the bloodstream (only several CTCs in each $1 \mathrm{ml}$ blood with 10 million leukocytes and 5 billion erythrocytes) remains a huge challenge in the clinical practice. ${ }^{132,133}$ Thus, isolation and enrichment of intact CTCs by the techniques, including immune-magnetic techniques, flow cytometry, microfluidic CTC chips, reverse transcription-polymerase chain reaction (RT-PCR), are general and necessary steps for sampling adequate CTCs prior to the detection. These techniques, however, are not very satisfying due to the limitations, including difficult blood sampling, time-consuming procedures, and high false positive rates. ${ }^{12,134-136}$ 
Photoacoustic (PA) imaging is a hybrid modality, combining the high sensitivity of optical imaging and the high resolution of acoustic imaging, and thus offers a unique opportunity to improve the early detection of cancer cells including the rare CTCs. In PA imaging, a short laser pulse is directed to an object to generate thermoelastic expansion, where the mechanical expansion releases by emitting acoustic waves that are detected by an ultrasonic transducer to generate imaging data. Thus, PA imaging is advantageous of desirable absorption contrast by the optical waves, as well as a high spatial resolution and penetration depth by ultrasonic waves. Hemoglobin and melanin are two typical endogenous chromophores in PA imaging that they display strong optical absorptions and acoustic wave emissions, and thus, the possibility of visualizing skin melanoma and tumor angiogenesis by PA imaging has been vastly explored and significant progress has been made in recent years. ${ }^{137-140}$ Label-free detection of melanoma CTCs can be realized by photoacoustic flow-cytometry $(\mathrm{PAFC}),{ }^{143-145}$ while for other types of cancer metastasis since there is a lack of endogenous contrast agent, exogenous contrast agents are necessary for highlighting small tumors and cancer cells including the CTCs from blood cell background.

Organic dyes and nanoparticles with optical absorption abilities are thought to be promising candidates as exogenous PA contrast agents to extend the applications of PA imaging. Organic dyes, as strong optical absorbers, are widely applied as imaging agents for fluorescence imaging, but most organic dyes are susceptible to photobleaching and are not hydrophilic. Furthermore, in the applications of PA imaging, there is an additional defect for organic dyes that most energy absorbed is 
converted to optical forms instead of acoustic forms, which is unfavorable to generate intense PA signals in living body. On the other hand, nanoparticles such as gold nanoparticles and quantum dots are promising candidates as the nanoparticle based PA contrast agents due to their strong optical absorption and high optic to acoustic conversion. ${ }^{37,39}$ However, due to the potential significant toxicity, the dosage of quantum dots allowed for imaging use is limited. Gold nanoparticles are advantageous of tunable and robust optical absorption, due to the surface plasmon resonance effect, but their bio-distribution, clearance, and potential toxicity are largely unknown, and further pre-clinical studies are necessary. ${ }^{141,142}$

This work aims to develop a silica coated iron oxide (SIO) nanoparticle decorated with Alexa Fluor ${ }^{\circledR} 750$ (AF750) dye and bombesin agonist peptide as a potent cancer cell selective PA contrast agent. Iron oxide nanoparticle (IO) features non-toxicity, biodegradability and has a history of medical applications. The superparamagnetism renders them to be robust contrast agents for MRI with large loading space for targeting molecules and other bio-conjugates. ${ }^{146-151}$ Although bare iron oxide (IO) nanoparticles are not strong infrared (IR) optical absorbers, the silica coated IO nanoparticle has shown to have a significant PA signal, as well as a stable architecture and accessibility to versatile surface bio-conjugations..$^{54,152}$ AF750 is an organic dye with the excitation peak at $749 \mathrm{~nm}$ and the emission peak at $775 \mathrm{~nm}$. Although most organic dyes are hydrophobic with a poor photostability, the conjugation of organic dyes to nanoparticles confers a possibility to improve the effectiveness of the organic dye as a PA contrast agent against the inherent defects. Bombesin (BBN), a 14 amino acid peptide, has 
attracted considerable interests as targeting moieties, due to the high binding affinity and selectivity to gastrin releasing peptide receptors (GRPrs) that are overexpressed in a variety of cancer types like pancreatic cancer, prostate cancer, small cell lung cancer, etc. ${ }^{102-104,106,107,109,110}$

In this work, BBN[7-14] $\mathrm{NH}_{2}$ was first conjugated with Alexa Fluor ${ }^{\circledast} 750$ (AF750) via a pharmacokinetic linker, then chemically connected with the functional groups on the surface of SIO nanoparticles to form SIO-AF750-BBN. This hybrid nanoparticle was purified and characterized and examined in PC-3 cells (a human prostate cancer cell line) for testing the specificity and binding affinity to the GRPrs. Finally, in vivo evaluation was performed in PC-3 tumor bearing mice to explore the in vivo binding affinity and specificity, as well as the PA imaging efficacy, pharmacokinetics, and biodistribution of the SIO-AF750-BBN nanoparticle.

\section{Materials and methods}

\section{Materials}

Lys-8Aoc-Gln-Trp-Ala-Val-Gly-His-Leu-Met-NH2 (K-8Aoc-BBN) was prepared using a conventional solid state peptide synthesis (SSPS) method from a commercial source (EZBiolab, Carmel, IN). Iron oxide nanoparticle with a core size of $10 \mathrm{~nm}$ was purchased from Ocean Nanotech (San Diego, CA). AF750 was purchased from Thermo Fisher Scientific (Waltham, MA). 1-Ethyl-3-(3-dimethylaminopropyl) carbodiimide (EDAC) was 
purchased from Sigma-Aldrich (St. Louis, MO). N-hydroxysulfosuccinimide (Sulfo-NHS) was bought from Fisher Scientific (Waltham, MA). Igepal, cyclohexane, 98\% Tetraethyl orthosilicate (TEOS), (3-Aminopropyl)triethoxysilane (APTES), succinic anhydride (SA), 28.0\%-30.0\% $\mathrm{NH}_{4} \mathrm{OH}$, and other regular chemicals $\left(\mathrm{NaHCO}_{3}, \mathrm{NaCl}\right.$, etc) and solvents (DMF, MES, butanol, isopropanol, ethanol, etc) were supplied by Sigma-Aldrich (St. Louis, MO).

\section{Modification of IO with Silica Coating}

$1.5 \mathrm{mg} \mathrm{IO}$ in $60 \mu \mathrm{l}$ chloroform was mixed with $880 \mu \mathrm{l}$ Igepal, $3.75 \mathrm{ml}$ cyclohexane and stirred for $30 \mathrm{~min}$. Then, $170 \mu \mathrm{l}$ Milli-Q water, $50 \mu \mathrm{l}$ TEOS (98\%) and $60 \mu \mathrm{l} \mathrm{NH}_{4} \mathrm{OH}$ (28.0-30.0\%) was added to the microemulsion, where the mixture was being stirred for $24 \mathrm{hr}$. At the end of the reaction, acetone was added to break the microemulsion, and the mixture was centrifuged $\left(4500 \mathrm{rpm}, 30 \mathrm{~min}, \mathrm{~T}=25^{\circ} \mathrm{C}\right)$ to form an oil phase and a water phase. Butanol, isopropanol, ethanol, and water were applied sequentially to wash away the surfactants and the unreacted molecules with a SuperMag separator (Ocean Nanotech, San Diego, CA). In between, re-dispersion of the precipitates may be accelerated with ultrasonic treatments. Eventually, the silica coated iron oxide nanoparticle (SIO) was dispersed in Milli-Q water. 


\section{Functionalization}

$0.36 \mathrm{mmol}$ APTES was mixed with $0.54 \mathrm{mmol}$ SA (pre-dissolved in $225 \mu \mathrm{l}$ DMF) for a reaction at room temperature for 8 hours. The obtained mixture was tested and the target APTES-SA was verified by a mass spectrum on an Agilent G6520A QTOF mass spectrometer with a Chip Cube source (G4240A) (Santa Clara, CA). No need to do any purification, the obtained $0.144 \mathrm{mml}$ APTES-SA in $90 \mu \mathrm{I}$ DMF was mixed with $0.6 \mathrm{mg}$ (per Fe content) SIO in $270 \mu \mathrm{l}$ water and $600 \mu$ l ethanol under $\mathrm{N}_{2}$ for a functionalization reaction at $50^{\circ} \mathrm{C}$ for 5 hours. The product was washed repeatedly with ethanol, water on the SuperMag separator. The final product was re-dispersed in water.

\section{Conjugation of AF750-K-8Aoc-BBN[7-14]NH2 (AF750-BBN)}

$1 \mathrm{mg}$ AF750 in $50 \mu \mathrm{l}$ DMF was mixed with $1.88 \mathrm{mg} \mathrm{K}-8 \mathrm{Aoc}-\mathrm{BBN}$ [7-14] $\mathrm{NH}_{2}$ in 200 $\mu \mathrm{l}$ DMF and $100 \mu \mathrm{l} 0.1 \mathrm{M} \mathrm{NaHCO}{ }_{3}$ buffer for a conjugation reaction at $4^{\circ} \mathrm{C}$ in the dark overnight. The product was then purified by a Shimadzu reverse phase highperformance liquid chromatography (RP-HPLC) (Kyoto, Japan) and analyzed on an Agilent G6520A QTOF mass spectrometer with a Chip Cube source (Santa Clara, CA).

Conjugation of AF750-K-8Aoc-BBN[7-14]NH2- with COOH Functionalized Silica coated IO Nanoparticle

$0.15 \mathrm{mg} \mathrm{COOH}$ functionalized SIO $(\mathrm{SIO}-\mathrm{COOH})$ in $0.125 \mathrm{ml}$ water was added MES and $\mathrm{NaCl}$ to make a $0.1 \mathrm{M} \mathrm{MES}$ and $0.5 \mathrm{M} \mathrm{NaCl}$ buffer solution, followed by adding $124 \mu \mathrm{g}$ 
EDAC and $110 \mu \mathrm{g}$ Sulfo-NHS for activation of $\mathrm{COOH}$ groups for 15 minutes (Ratio of EDAC and Sulfo-NHS to AF750-BBN-NH 2 is $50 / 40$ to 1). $26.9 \mu \mathrm{g}$ AF750-K-8Aoc-BBN was then added to the solution stirring at $4^{\circ} \mathrm{C}$ and dark overnight (experimental design is 100 AF750-BBN per each nanoparticle). The product was purified using a $100 \mathrm{kDa}$ dialysis membrane three times for 24 hours.

\section{FTIR}

To demonstrate the existence of silica coating on the 10 nanoparticle after the first step of the reaction, Fourier transform infrared (FTIR) spectra were measured on a Mattson Galaxy series 5000 FTIR spectrometer (Madison, WI). Each spectrum in the middle infrared range $(4000 \mathrm{~cm}-1$ to $400 \mathrm{~cm}-1)$ has an average of 16 scans with the spectral resolution set to be $2 \mathrm{~cm}^{-1}$. The FTIR samples were prepared following the $\mathrm{KBr}$ method in which a small quantity of dry sample was mixed with dry $\mathrm{KBr}$ powder, and the mixture was subjected to a press at several tons for about 2 min to generate a sample pellet.

TEM

The morphology and dimension of the nanoparticles in different stages of the reaction were examined and measured on a JEOL 1400 transmission electron microscope (TEM) with accelerating voltage of 40-120 kV (Tokyo, Japan). For the sample 
preparation, the nanoparticle solution was first broken up in a sonicator for 3 minutes, and then $20 \mu \mathrm{l}$ was drawn and dripped onto a piece of carbon coated copper grids. The Incubation was allowed for 5 minutes, and each piece of grids was wiped and air dried before being inserted into the specimen chamber of the TEM instrument.

\section{Determination of iron content of nanoparticles}

The iron concentration in SIO nanoparticles was determined using Prussian blue staining spectrophotometric method. Standard solutions of different iron concentrations $(0.112,0.0896,0.0672,0.0448,0.0224,0 \mathrm{mg} / \mathrm{ml})$ were prepared using Ferridex (Bayer, Leverkusen, Germany). Each $200 \mu \mathrm{L}$ solution was added with $200 \mu \mathrm{L}$ 12.1 $\mathrm{N} \mathrm{HCl}$ for acid hydrolysis reaction at $80^{\circ} \mathrm{C}$ for 4 hours, followed by adding $400 \mu \mathrm{L}$ MilliQ water for dilution and $200 \mu \mathrm{L}$ 5\% Prussian blue for staining. The absorbance of each solution was determined at $690 \mathrm{~nm}$ on a Shimazu $1601 \mathrm{UV}$-vis spectrophotometer

(Kyoto, Kyoto Prefecture, Japan). A standard correlation of the light absorbance versus iron concentration was fitted to a linear curve according to the Beer's Law:

$$
\text { Absorbance }=\log \left(I_{0} / I\right)=\varepsilon L C
$$

Where $I_{0}$ and $I$ are the light intensity before and after passing the solution, $\varepsilon$ is the molar extinction coefficient (or molar absorptivity constant), $L$ is the path length of the sample cuvette, and $\mathrm{c}$ is the concentration of the solution. To prepare the sample solution, $20 \mu \mathrm{L}$ stock solution was diluted to $200 \mu \mathrm{L}$ with Milli-Q water and mixed with 
$200 \mu \mathrm{L} 12.1 \mathrm{~N} \mathrm{HCl}$ for $4 \mathrm{hrs}$ at $80^{\circ} \mathrm{C}$, followed by addition of $400 \mu \mathrm{L}$ water and $200 \mu \mathrm{L} 5 \%$ Prussian blue solution. The light absorption was also measured on the Shimazu 1601 UV-vis spectrophotometer. The iron concentration was estimated against the standard curve with triplicate measurements.

\section{Determination of peptide to nanoparticle ratio}

First, standard solutions of AF750 in $100 \mu \mathrm{L}$ water at different concentrations (0, $0.03125,0.0625,0.125,0.25,0.5$ and $1 \mathrm{ug} / \mathrm{ml}$ ) were prepared in a 96-well Cellstar transparent microplate for the fluorescence measurement on a Synergy $\mathrm{H} 4$ hybrid reader (Biotek, Winooski, VT) with excitation of $740 \mathrm{~nm}$ and emission of $780 \mathrm{~nm}$. The fluorescence intensities versus concentrations were fitted to a linear curve based on the Beer's Law as well. The concentration of AF750-BBN in the nanoparticle solution was estimated against the standard curve of AF750 fluorescence intensity versus concentration. The iron content of SIO-AF750-BBN was obtained by the Prussian blue staining method, and the molar concentration of the SIO nanoparticles was estimated according to the information regarding the ratio of iron content to the molar concentration of nanoparticles given by the supplier. The peptide to nanoparticle ratio was then calculated using the molar concentration of AF750-BBN to be divided by the molar concentration of iron oxide nanoparticles. 


\section{MRI relaxivity Measurement}

The solutions of SIO-AF750-BBN and Feridex were prepared in saline at $\mathrm{pH}$ 7.4. $\mathrm{R}_{2}$ relaxation rates were determined at iron concentrations of $0.1,0.05,0.025,0.0125$ and $0.00625 \mathrm{mM}$. The measurements were repeated on two or more independently prepared samples to ensure consistency. A buffer matched blank sample $(0 \mathrm{mM})$ was also used in the relaxivity measurements of each sample. Measurements were performed using a 7 Tesla Bruker BioSpec Avancelll MRI system (Bruker BioSpin, Corporation) equipped with a volume radiofrequency (RF) coil (35 mm inner diameter) at $25{ }^{\circ} \mathrm{C} . \mathrm{R}_{2}$ was measured using a T2-Map-MSME pulse sequence with slice thickness $=$ $1 \mathrm{~mm}$, matrix $=256 \times 256, \mathrm{FOV}=2.60 \times 2.60 \mathrm{~mm}, \mathrm{TE}=9.5 \mathrm{~ms}$ to $237.5 \mathrm{~ms}$ with an interval of 9.5, and TR = 2000 ms. Signal intensity of every sample was measured, and the relaxation rate was obtained by the exponential fitting functions in the ParaVision 6.0 software (Bruker BioSpin Corporation, 2012). The final relaxivity value was derived from a linear fitting equation of the relaxation rates against the different concentrations of the nanoparticle samples, $R_{2}=R_{20}+r_{2} * c$, where the $r_{2}$ is the relaxivity, $c$ is the concentration, $R_{2}$ and $R_{20}$ are the relaxation rates of the solution at the concentration $c$ and 0 , respectively.

\section{Measurements of Photoacoustic Strength}

The ability to generate photoacoustic signals in a broad wavelength range was evaluated on a Vevo 2100/LAZR photoacoustic imaging system (FUJIFILM VisualSonics 
Inc., Toronto, Canada). In this study, Feridex (clinically approved iron oxide nanoparticle based MRI T2 contrast agent) and casein coated iron oxide nanoparticle (the product of Chapter 3) were used as the references. The sample was prepared in a plastic capillary tube, placed on a gel that is touched by the ultrasonic transducer of the imaging system. Scanning range was set from 680nm to $970 \mathrm{~nm}$.

\section{In Vitro Experiment}

Human prostate cancer (PC-3) cell-lines were bought from American Type Culture Collection (ATCC, Manassas, VA) and maintained by the Cell and Immunobiology Core Facility at the University of Missouri. RPMI 1640 was purchased from Thermo Fisher Scientific (Waltham, MA), combined with $10 \%$ fetal bovine serum (FBS) from Sigma-Aldrich (St. Louis, MO) and $1 \%$ penicillin from Invitrogen (Carlsbad, CA) as the cell culture medium. Trypsin with $0.25 \%$ EDTA from Invitrogen (Carlsbad, CA) was used to suspend cells. Forma water-jacketed incubator, manufactured by Fisher Scientific (Waltham, MA) offered proper growing environment $\left(37^{\circ} \mathrm{C}\right.$ and $\left.5 \% \mathrm{CO} 2\right)$ for $\mathrm{PC}-3$ cells.

The specificity of the compounds' binding to prostate cancer cells was evaluated through an uptake study and a blocking study in PC3 cells. In the uptake study, 2 million PC-3 cells were incubated with $0.00118 \mathrm{mg}$ (in Fe) SIO-AF750-BBN for 50 minutes in the incubator. While in the blocking study, 2 million PC-3 cells from the same cell line were pre-incubated with $22.3 \mu \mathrm{g} B B N[1-14]$ as the blocking agent before the incubation with the same amount of SIO-AF750-BBN for 50 minutes in the incubator. The PC-3 cells were 
then washed with the cold cell culture medium and PBS repeatedly. Eventually, a small amount of PBS was left in the test tube to mix with the cell pellet and $10 \mu$ l solution was drawn to drop onto a glass slide for observations on the Carl Zeiss Axiovert 200M inverted microscope (Oberkochen, Germany) equipped with a Cy7 filter set, whose excitation wavelength ranges from 673-748 $\mathrm{nm}$ and emission wavelength ranges from $765-835 \mathrm{~nm}$. All procedures were performed in the dark.

\section{Animal model}

In vivo studies were performed in severely compromised immunodeficient (SCID) mice bearing human PC-3 prostate cancer xenografts. Animal studies were conducted in accordance with the highest standards of care as outlined in the NIH's guide for care and use of laboratory animals and in accordance with policy and procedures for animal research at the Harry S. Truman Memorial Veterans Hospital. Four-to-five-week-old SCID mice were obtained from Taconic (Germantown, NY). Mice were housed four animals per cage in sterile micro-isolator cages in a temperature- and a humiditycontrolled room with a 12-hour light/dark schedule. The animals were fed with autoclaved rodent chow (Ralston Purina Company, St. Louis, MO) and provided with water ad libitum. Mice were inoculated with $7 \times 10^{6}$ PC-3 tumor cells in $0.1 \mathrm{ml}$ matri-gel on each right and left flank. Six mice ( $n=3$ for uptake and $n=3$ for blocking) were used in NIRF imaging, MRI, and PA experiments between 3-4 weeks post inoculation of tumor cells. Average body weight of mice was 25-30 grams at the time of the study. 


\section{In Vivo MRI}

MRI study of tumor-bearing animals was performed on a 7 T Bruker AVANCE III BioSpec MRI scanner (Bruker BioSpin Inc., Billerica, MA) equipped with a gradient insert (400 mT/m, $115 \mathrm{~mm}$ I.D.) and a quadrature RF coil (35 mm ID). Mice were anesthetized with $1-2 \%$ isoflurane in oxygen via a nose cone over the entire imaging period. A respiratory sensor was placed on the abdomen for respiratory monitoring of vital signs using a Physiological Monitoring System (SA Instruments, Inc.; Stony Brook, NY). Body temperature was maintained at $37^{\circ} \mathrm{C}$ with warm air circulating in the magnet bore. A T2map-MSME imaging sequence was used to obtain the T2 weighted images, and a T2 relaxation parametric imaging sequence was applied to obtain the T2 relaxation time for different regions of interest. For T2-map-MSME imaging sequence: $\mathrm{TR}=4694.9 \mathrm{~ms}$, TE $=$ $15 \mathrm{~ms}$ to $225 \mathrm{~ms}$ with an interval of $15 \mathrm{~ms}, 14$ slices, $\mathrm{ST}=1 \mathrm{~mm}$ with no gap, matrix = $256 \times 128, \mathrm{FOV}=27 \times 22 \mathrm{~mm}$ (axial), number of excitations $(\mathrm{NEX})=2$, acquisition time 20 minutes. After the baseline pre-injection scan was completed, a dose of $55.0 \mu \mathrm{g}$, $64.3 \mu \mathrm{g}$, or $93.0 \mu \mathrm{g}(\mathrm{Fe})$ of SIO-AF750-BBN in $100 \mu$ isotonic saline was injected as a bolus via tail vein as the uptake groups and the same dosage combined with a pre-injection of $75 \mu \mathrm{g} B \mathrm{BN}[1-14]$ in $75 \mu \mathrm{l}$ water was applied for the mice as the blocking group. The MRI images were obtained at 2 hours after injection and compared with the pre-injection MRI.

Image analysis and processing were performed using ParaVision 6.0 software (Bruker BioSpin Corporation, 2012). Regions of interest (ROIs) were manually drawn on the tumor tissue, muscle near the tumor for each time point. Signal intensity (SI) was 
measured as the mean of the intensity over the segmented ROI. The relaxation time for each $\mathrm{ROI}$ was directly read from the $\mathrm{T} 2$ relaxation parametric images.

\section{In vivo PA imaging}

The same mice in the MRI study were used for this study with the imaging time at 1-hour post injection. The PA imaging was evaluated on a Vevo 2100/LAZR photoacoustic imaging system (FUJIFILM VisualSonics Inc., Toronto, Canada) equipped with a transducer LZ250 (13 - $24 \mathrm{MHz})$. The mice were maintained at 1-2\% isoflurane for anesthesia and kept warm throughout the study. The tumor and surrounding area were gently shaved. The tumors were examined by applying the ultrasound gel and the ultrasonic transducer at the scanning range set from $680 \mathrm{~nm}$ to $970 \mathrm{~nm}$ wavelength. Three additional mice were injected with a small molecule AF750-Gpip-Sta-BBN (2 uptakes and 1 blocking) and acquired the PA images under the same parameter setting.

\section{In vivo NIRF imaging}

In vivo and ex vivo NIRF imaging was performed on an IVIS Spectrum imaging system (PerkinElmer, Waltham, MA) equipped with a cooled charge-coupled device (CCD) camera and a 150-W quartz halogen light source. The NIRF images were acquired at a filter setting (excitation $745 \mathrm{~nm}$; emission $800 \mathrm{~nm}$ ) with the parameters: exposure time (2 sec), f/stop (4), binning (M)8, and field of view $(13.2 \mathrm{~cm})$. Fluorescence semi- 
quantification was performed using Living Image 4.4 (Xenogen). Region-of-interests (ROIs) were drawn on the tumors and muscles as the background in the NIRF images, and the expression of fluorescence emission intensity was normalized to average radiant efficiency $\left([\mathrm{p} / \mathrm{sec} / \mathrm{cm} 2 / \mathrm{sr}] /\left(\mu \mathrm{W} / \mathrm{cm}^{2}\right)\right)$.

After the baseline scan had been completed, the same set of mice in the MRI study were used for this study with the imaging time at $15 \mathrm{~min}, 0.5 \mathrm{hr}, 1 \mathrm{hr}, 2 \mathrm{hr}, 3 \mathrm{hr}$ and 4hr post injection. After the time point at 4 hours post injection (for only one group of mice the sacrifice time was at 3.5 hours post injection), mice were sacrificed by cervical dislocation under anesthesia ( $4 \%$ isoflurane in $1 \mathrm{~L} / \mathrm{min} \mathrm{O}_{2}$ ). Organs were collected and were subjected to the following ex vivo NIRF imaging.

\section{Histopathology}

Tumors, prostate, pancreas, and muscles of the mice from the in vivo studies were fixed in $10 \%$ neutral-buffered formalin and then were embedded in paraffin blocks, and slices of $5 \mu \mathrm{m}$ thickness were made on a microtome. Tissues were stained with Prussian blue (potassium ferrocyanide 10\%/ $\mathrm{HCl} 20 \%(\mathrm{v} / \mathrm{v})$ ) and nuclear fast red for tumor specimens. The images were taken using a Leica DM5500B microscope (Wetzlar, Germany). 


\section{Statistical analysis}

Quantitative data were expressed as the mean \pm SD. Means were compared by analysis of a student's $t$-test. $P$ values of less than 0.05 were considered to be statistically significant.

\section{Result}

\section{Synthesis and Characterization}

SIO-AF750-BBN was synthesized from $10 \mathrm{~nm}$ iron oxide nanoparticles, followed by the steps of silica coating with TEOS, $\mathrm{COOH}$-functionalization with APTES-SA, and labeling with K-8Aoc-BBN-AF750. The whole process was illustrated in Figure 4.1. 


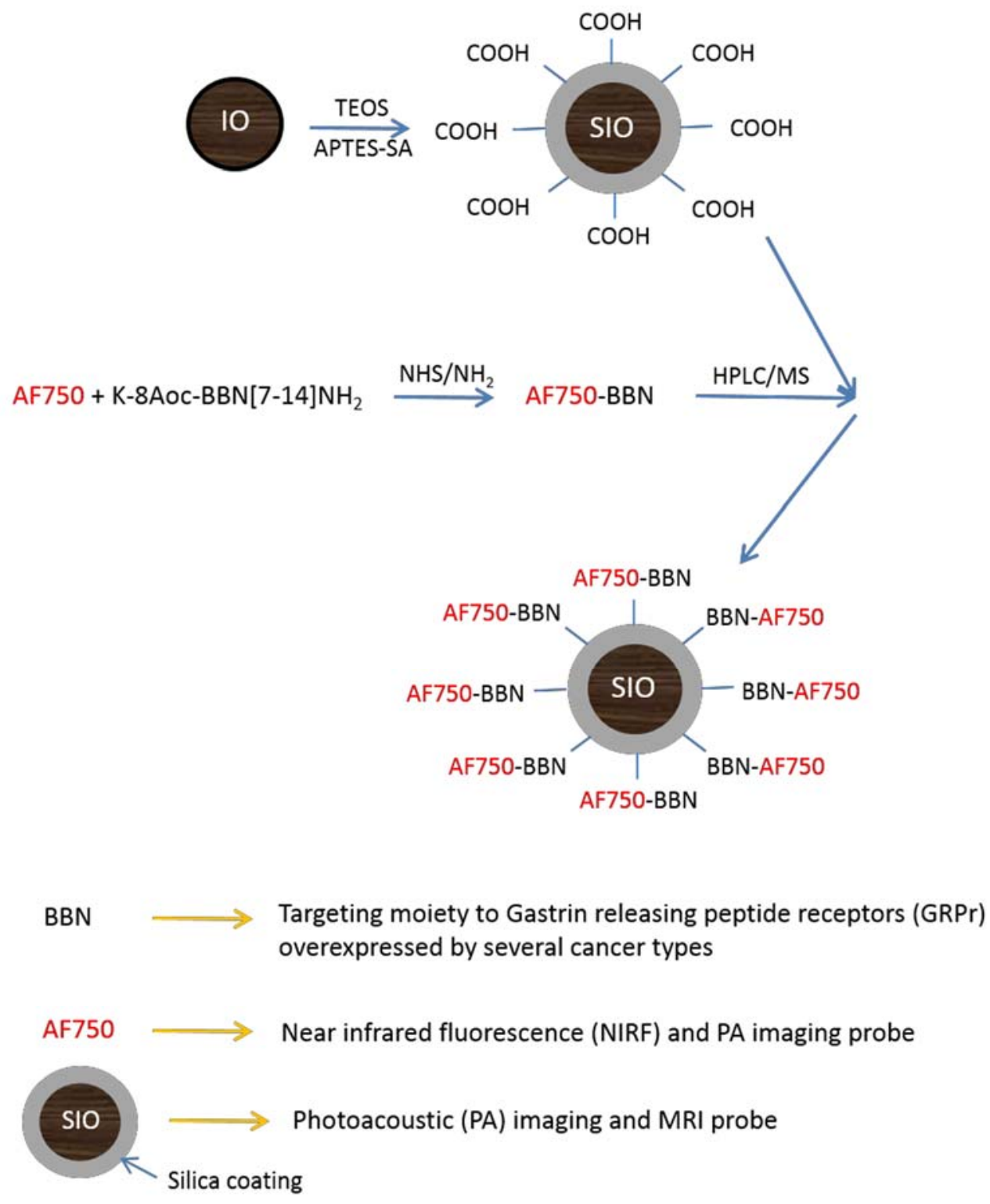

Abbreviations: IO: iron oxide nanoparticle TEOS: Tetraethyl orthosilicate APTES: (3-Aminopropyl)triethoxysilane SA: succinic anhydride SIO: silica coated iron oxide nanoparticle BBN: Bombesin AF750: Alexa Fluor 750

Figure 4.1 Schematic diagram of silica coating, functionalization, and conjugations to obtain SIO-AF750-BBN.

AF750-K-8Aoc-BBN[7-14]NH . AF750-BBN (Figure 4.2) was conjugated via EDAC/NHS chemistry, purified to $>95 \%$ by HPLC (Figure $4.3 \mathrm{~A}$ ), and confirmed by mass 
spectrometry (Figure 4.3B). Its experimental molecular weight (MW) is $2075.91 \mathrm{Da}$, matching the theoretical value of 2075.79 Da as shown in Figure 4.3.

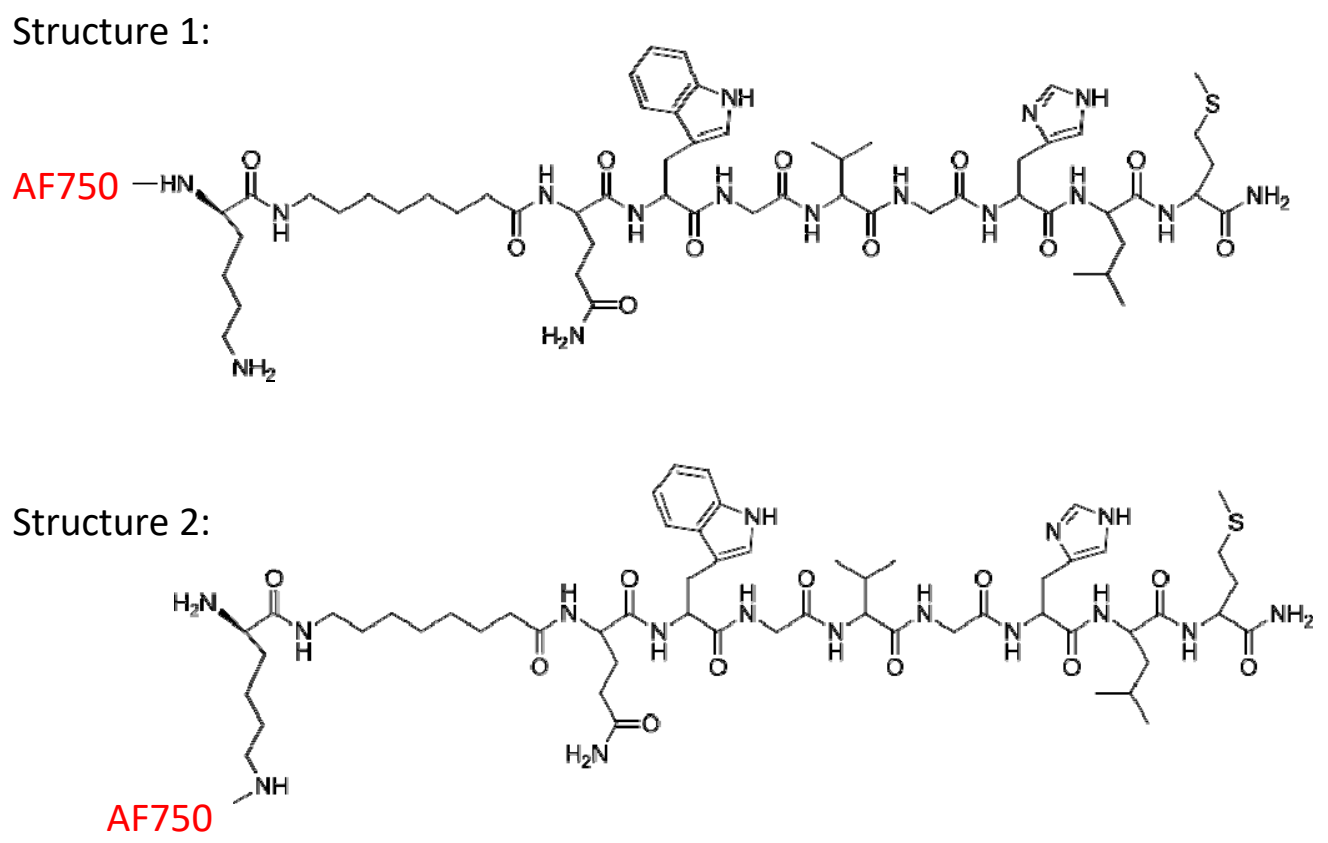

Figure 4.2 Molecular structures of AF750-K-8Aoc-BBN[7-14]NH2 (AF750-BBN). Only one of the two $\mathrm{NH} 2$ on lysine is conjugated with AF750. 

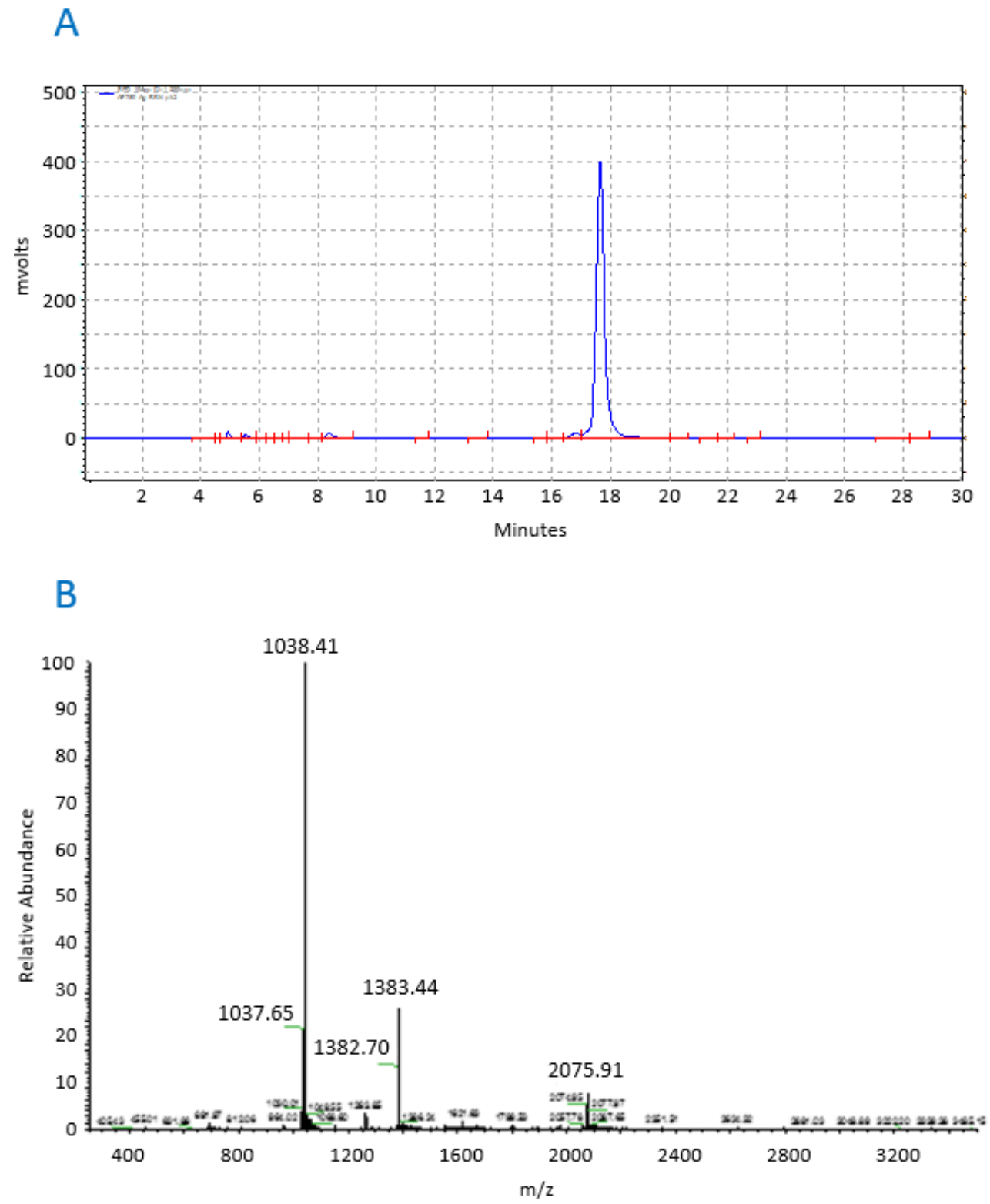

Figure 4.3(A) HPLC profile (retention time: $17.7 \mathrm{~min}$ ) and (B) mass spectrum of AF750-K8Aoc-BBN[7-14] $\mathrm{NH}_{2}$ (AF750-BBN). Measured MW is $2075.9 \mathrm{Da}$, in good agreement with the theoretical MW of 2075.8 Da.

Silica coating and $\mathrm{COOH}$ functionalization. The existence of the silica coating was validated by FTIR. As shown in Figure 4.4 the $\mathrm{Si}-\mathrm{O}$ bond is located at $1088 \mathrm{~cm}^{-1}$ confirming the conjugation of silicon oxide on the 10 nanoparticle. To functionalize the silica coated iron oxide nanoparticles with $\mathrm{COOH}$, APTES-SA was prepared by reaction of 
APTES and SA in DMF and was used to modify the SIO surface with COOH. The APTES-SA product was also confirmed by mass spectroscopy (Figure 4.5). The result showed that the sodium adduct has a molecular weight of $344.16 \mathrm{Da}$ which is equal to $321.17 \mathrm{Da}$ after deducting one sodium, matching the theoretical molecular weight of APTES-SA, 321.44Da. After the $\mathrm{COOH}$ functionalization and purification, the $\mathrm{pH}$ value of the $\mathrm{SIO}$ nanoparticle solution changed from 7.07 to 5.26 , revealing that the addition of $\mathrm{COOH}$ groups reduced the $\mathrm{pH}$ value of the solution.

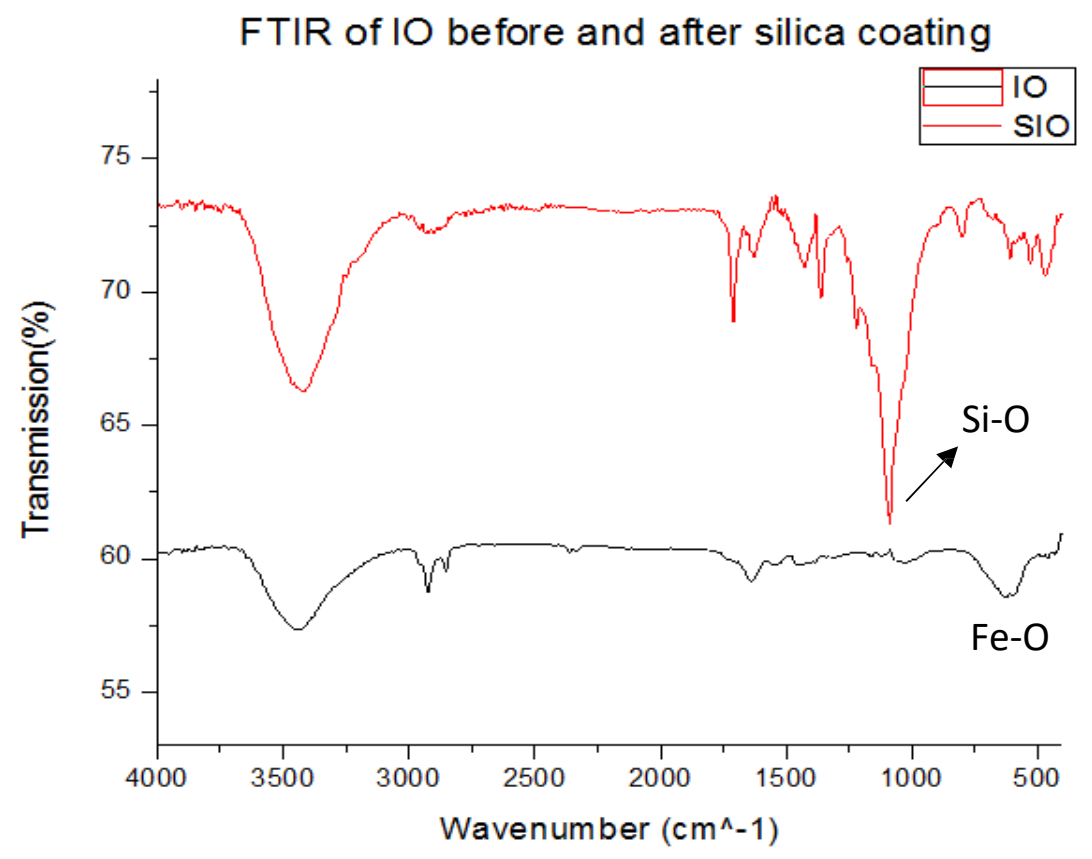

Figure 4.4 FTIR spectra of IO nanoparticle before and after silica coating 


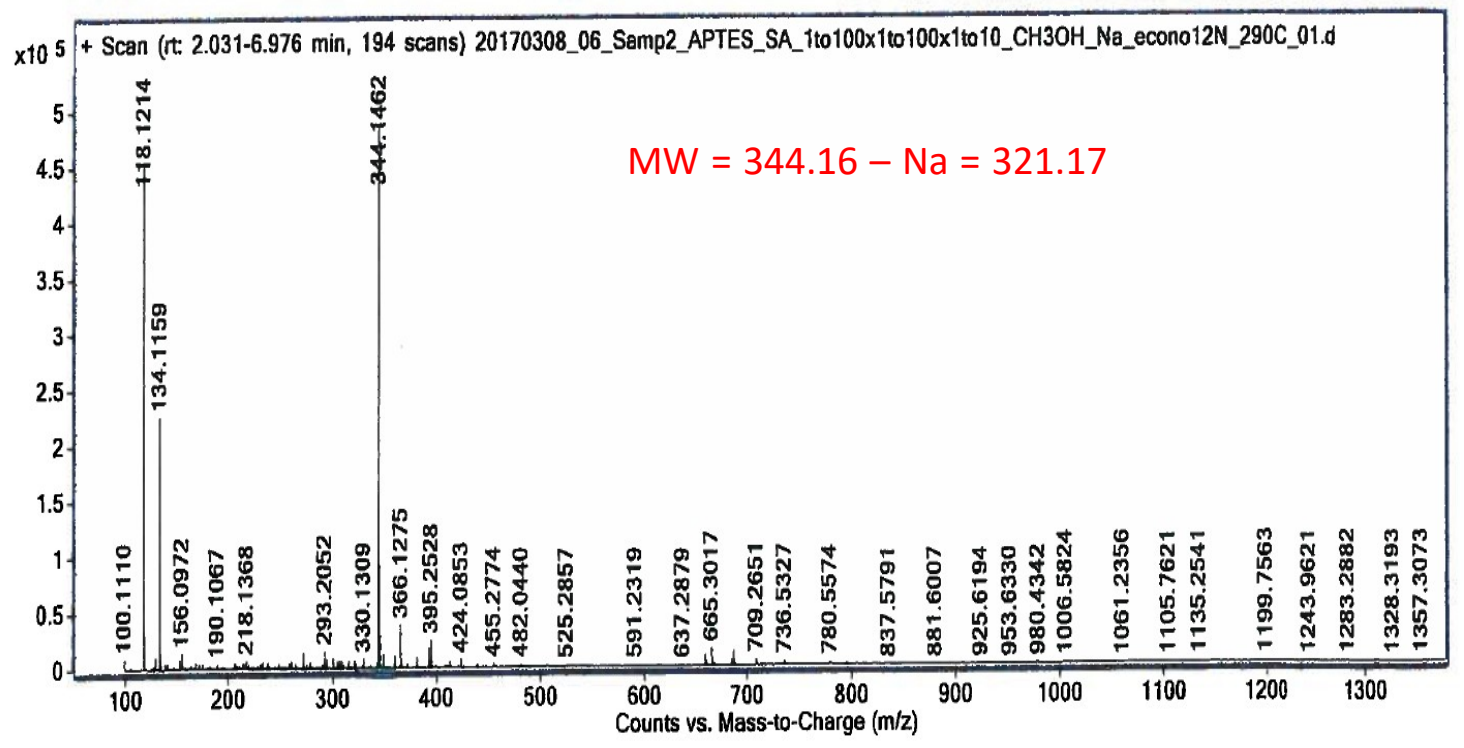

Figure 4.5 Mass spectroscopy of APTES-SA
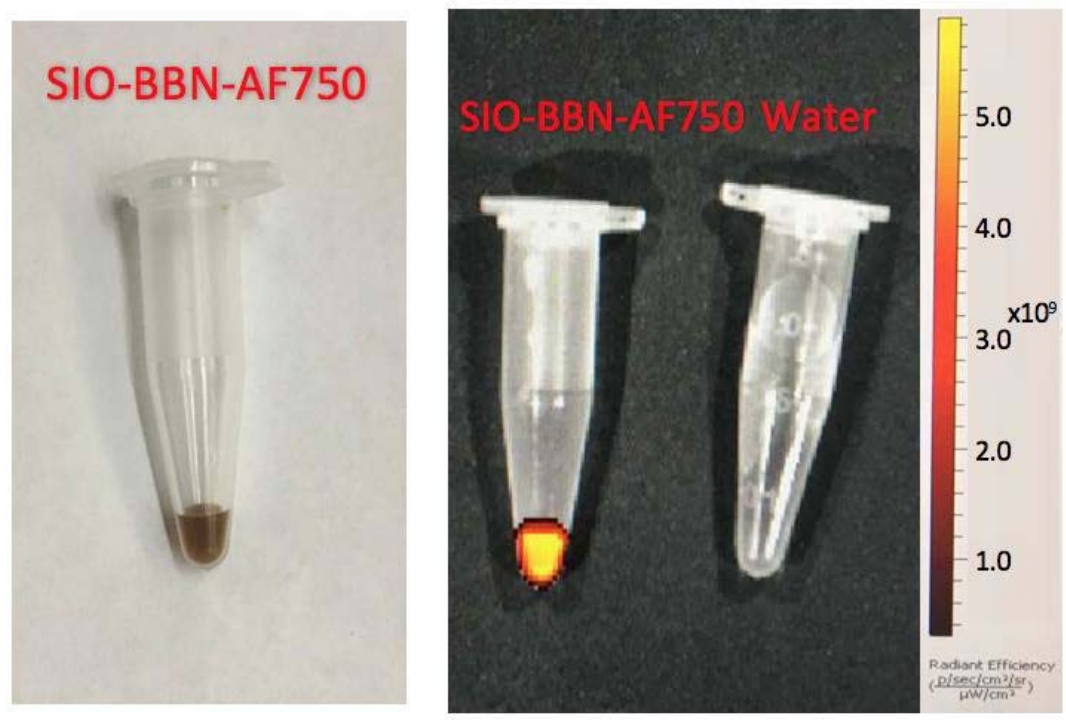

Figure 4.6 SIO-AF750-BBN and its NIR fluorescence imaging on an IVIS system. 
SIO-AF750-BBN. The purified final compound, SIO-AF750-BBN, is water soluble and a dark brown solution and displays strong fluorescent intensities in IVIS imaging (Figure 4.6). The TEM images (Figure 4.7) exhibit the morphologies and dimensions of the SIO, $\mathrm{COOH}$ functionalized $\mathrm{SIO}(\mathrm{SIO}-\mathrm{COOH})$ and the final SIO-AF750-BBN nanoparticles. The silica coated iron oxide nanoparticle (Figure 4.7A) has a silica coating, displayed as the gray coating on the IO core. The SIO-AF750-BBN (Figure 4.7C) exhibits a core diameter of $9.72 \mathrm{~nm} \pm 0.69$ and a silica coating thickness of $7.45 \pm 0.88 \mathrm{~nm}$. The functionalization reaction and the conjugation with AF750-BBN did not alter the thickness of the silica coating and the core materials. 


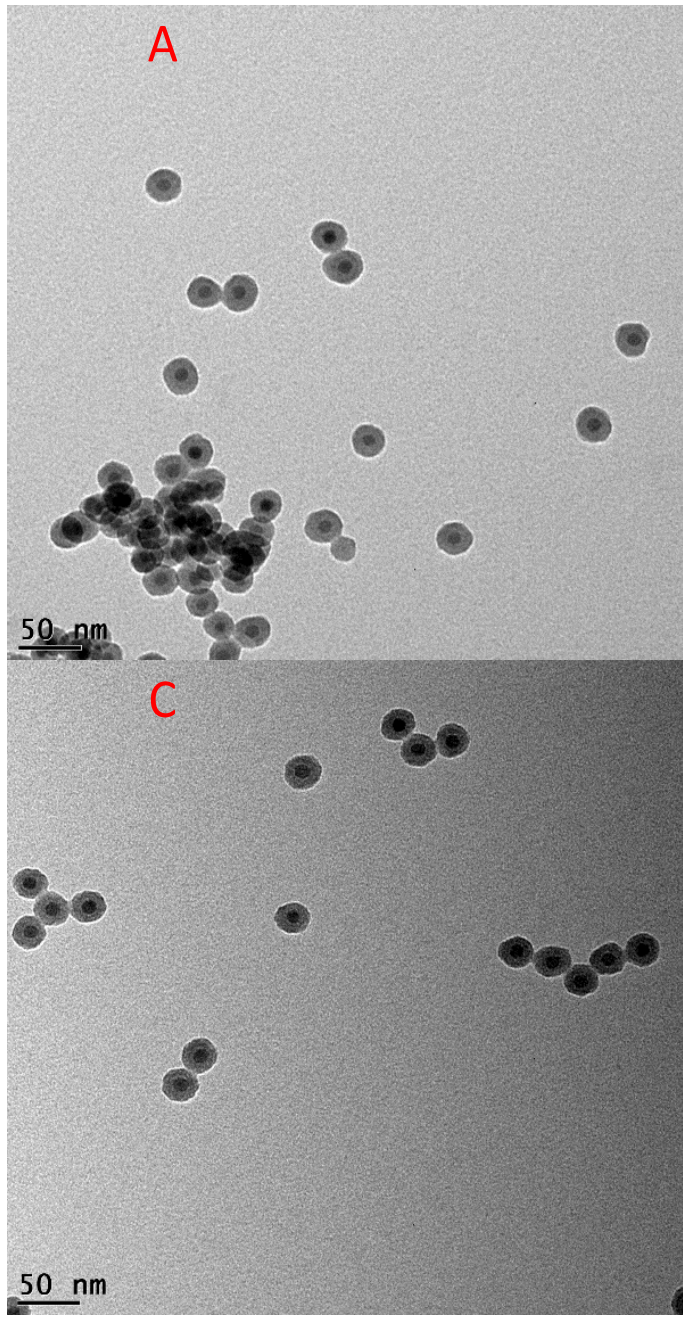

B

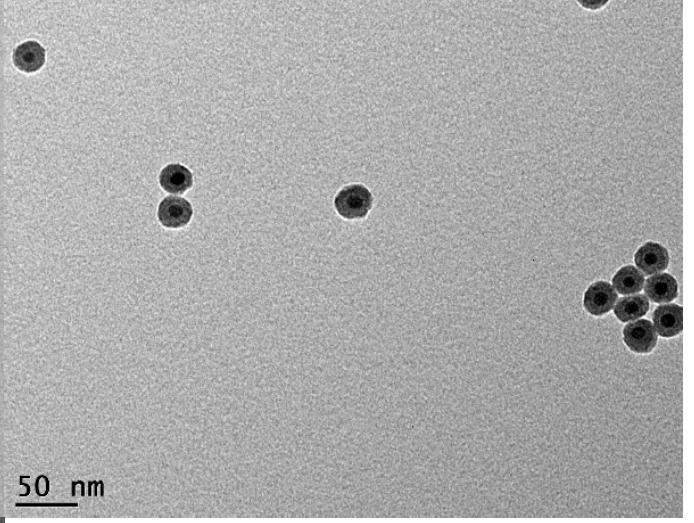

D

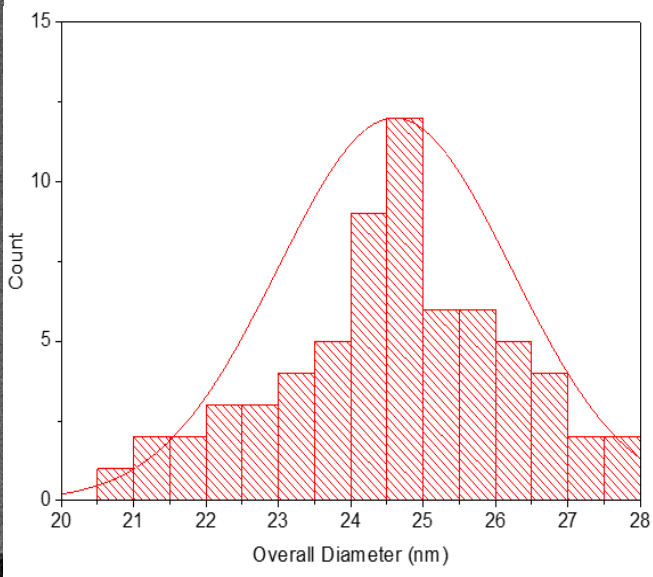

Figure 4.7 TEM images of (A) silica coated iron oxide nanoparticles and (B) $\mathrm{COOH}$ functionalized silica coated iron oxide nanoparticle (C) SIO-AF750-BBN (D) Histogram of the overall diameters of SIO-AF750-BBN

Figure 4.8 shows the T2-weighted MRI images of SIO-AF750-BBN at varies concentrations as compared to Feridex ${ }^{\circledR}$ and their $r_{2}$ relaxivity measurements. The $r_{2}$ relaxivity of SIO-AF750-BBN is $210.35 \mathrm{mM}^{-1} \mathrm{~s}^{-1}$, higher than that of Feridex, a clinically approved MRI contrast agent whose $r_{2}$ relaxivity is $156.29 \mathrm{mM}^{-1} \mathrm{~s}^{-1}$. 
A

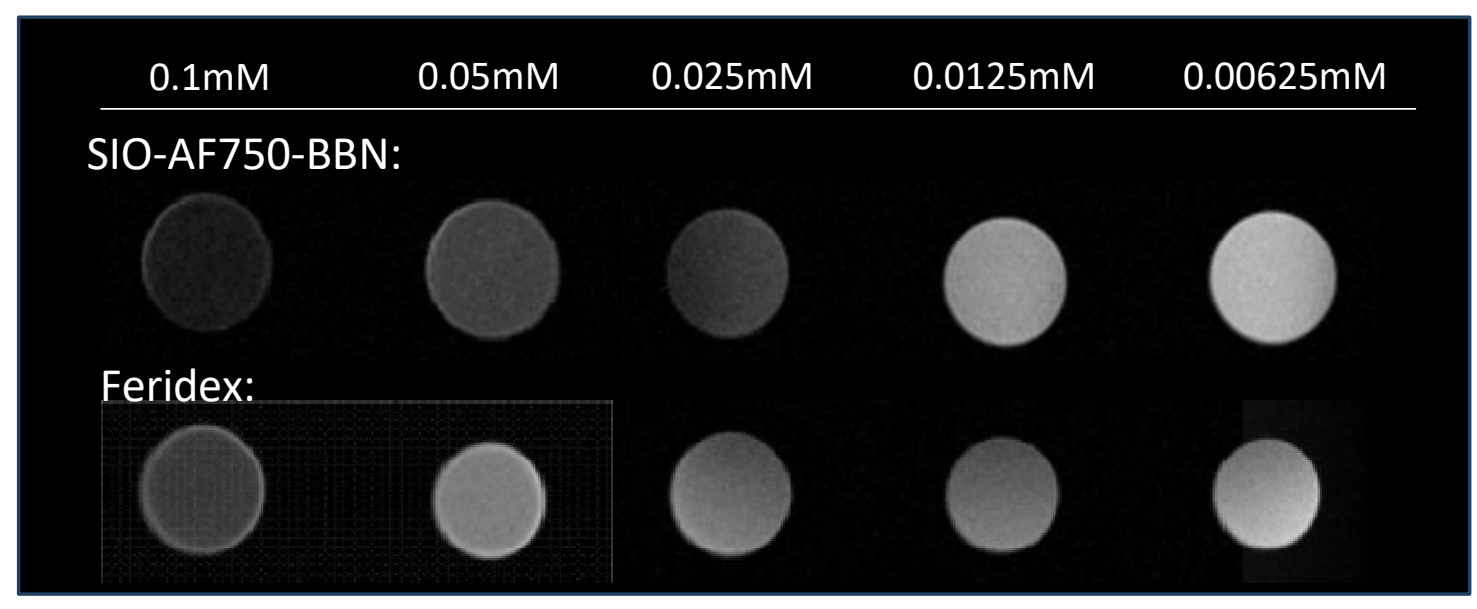

B

C
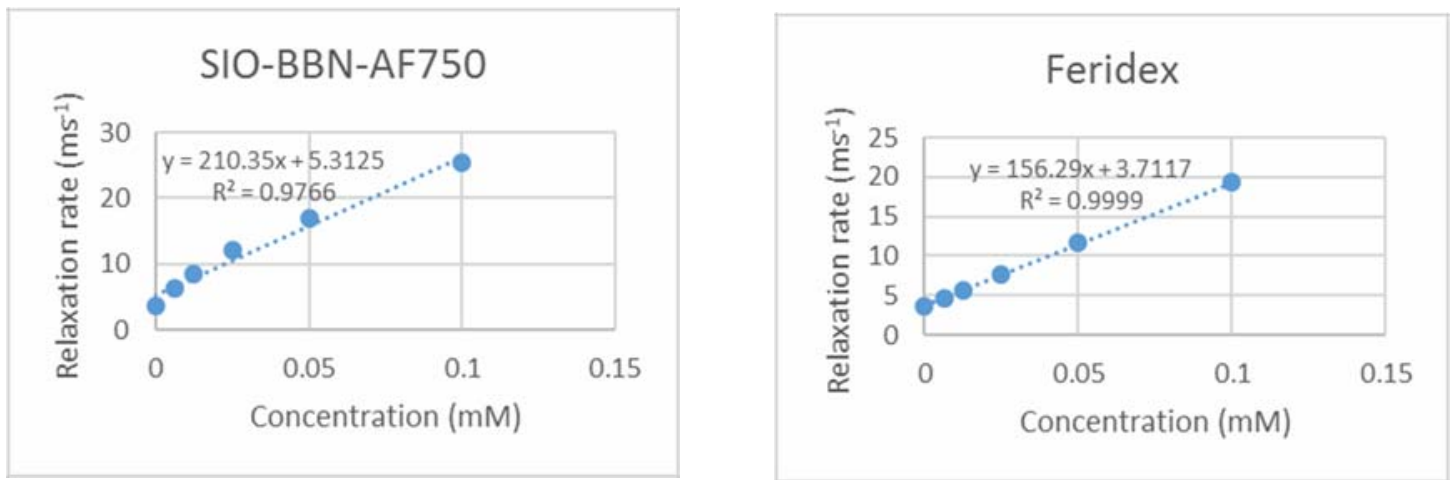

Figure 4.8 $\mathrm{MRI} r_{2}$ relaxivity measurements of SIO-AF750-BBN and Feridex ${ }^{\circledast}$. (A) T2weighted MRI at varies concentrations, and $r_{2}$ fittings of (B) SIO-AF750-BBN and (C) Feridex ${ }^{\circledR}$.

PA strength measurements for SIO, USPIO(Cy7.5)-BBN (the compound in Chapter 3 ), and Feridex ${ }^{\circledR}$ (commercial product) were made to characterize the potential of the compound in optical absorption, as well as conversion efficiency of optical absorption to acoustic energy. After normalization of concentration, as shown in Figure 4.9, the SIO nanoparticle was revealed to have more than 2 times the PA intensity as compared to Feridex, and about 14 times the USPIO(Cy7.5)-BBN at excitation wavelength of $925 \mathrm{~nm}$, 
proving the photoacoustic signal enhancement by the silica coating to the iron oxide nanoparticles.
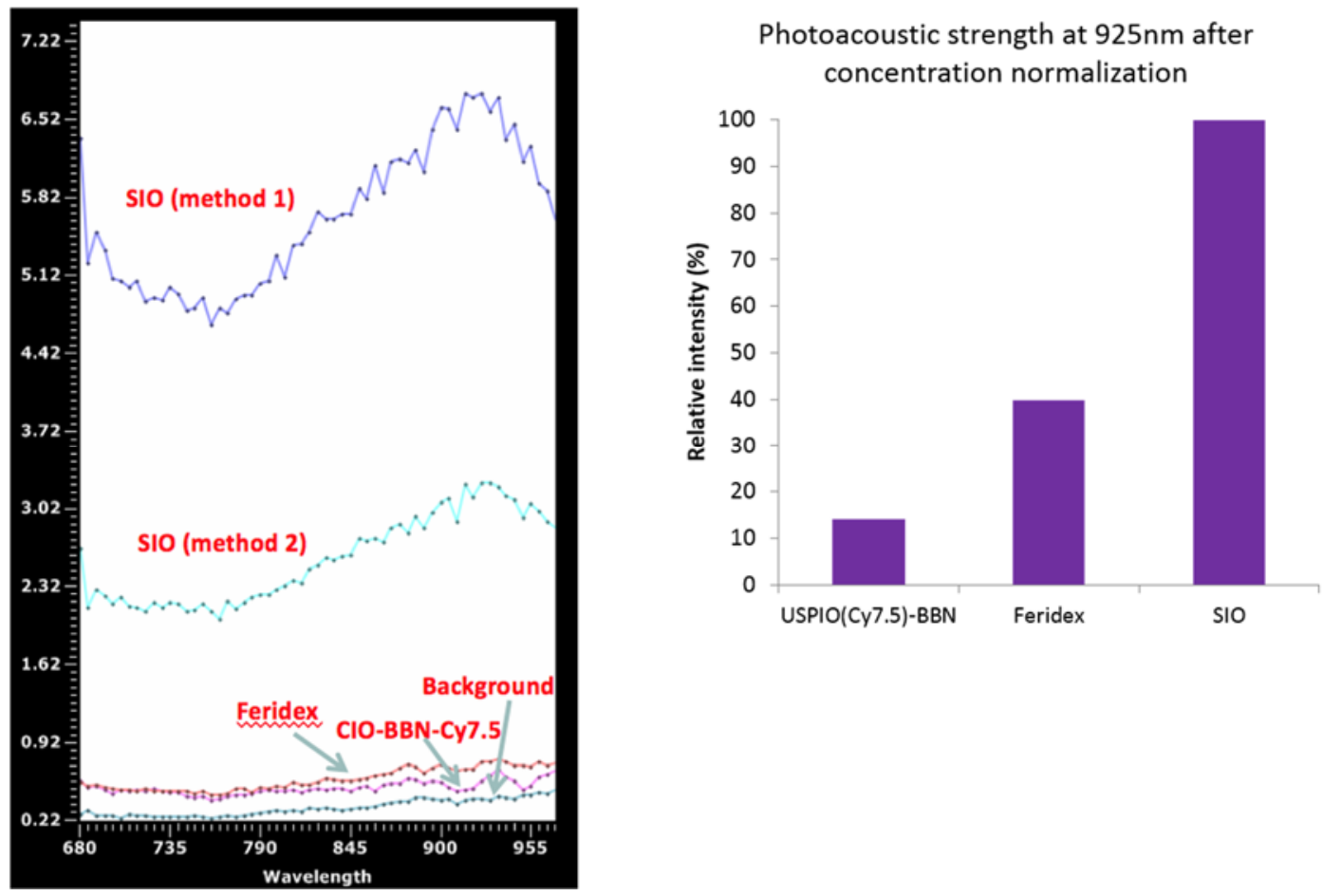

Figure 4.9 PA intensity of SIO as compared with USPIO(Cy7.5)-BBN and Feridex as references. The chart on the right displays the relative PA intensity of different compounds after concentration normalization.

In vitro evaluation of SIO-AF750-BBN. As Figure 4.10 displays, the PC-3 cells in the uptake group demonstrated high uptake of fluorescence, but there is no or little cellular uptake of fluorescence in the blocking group with the unlabeled BBN[1-14] as the blocking agents. This result indicated that SIO-AF750-BBN could specifically bind to the GRP receptors overexpressed on the PC-3 cells. 


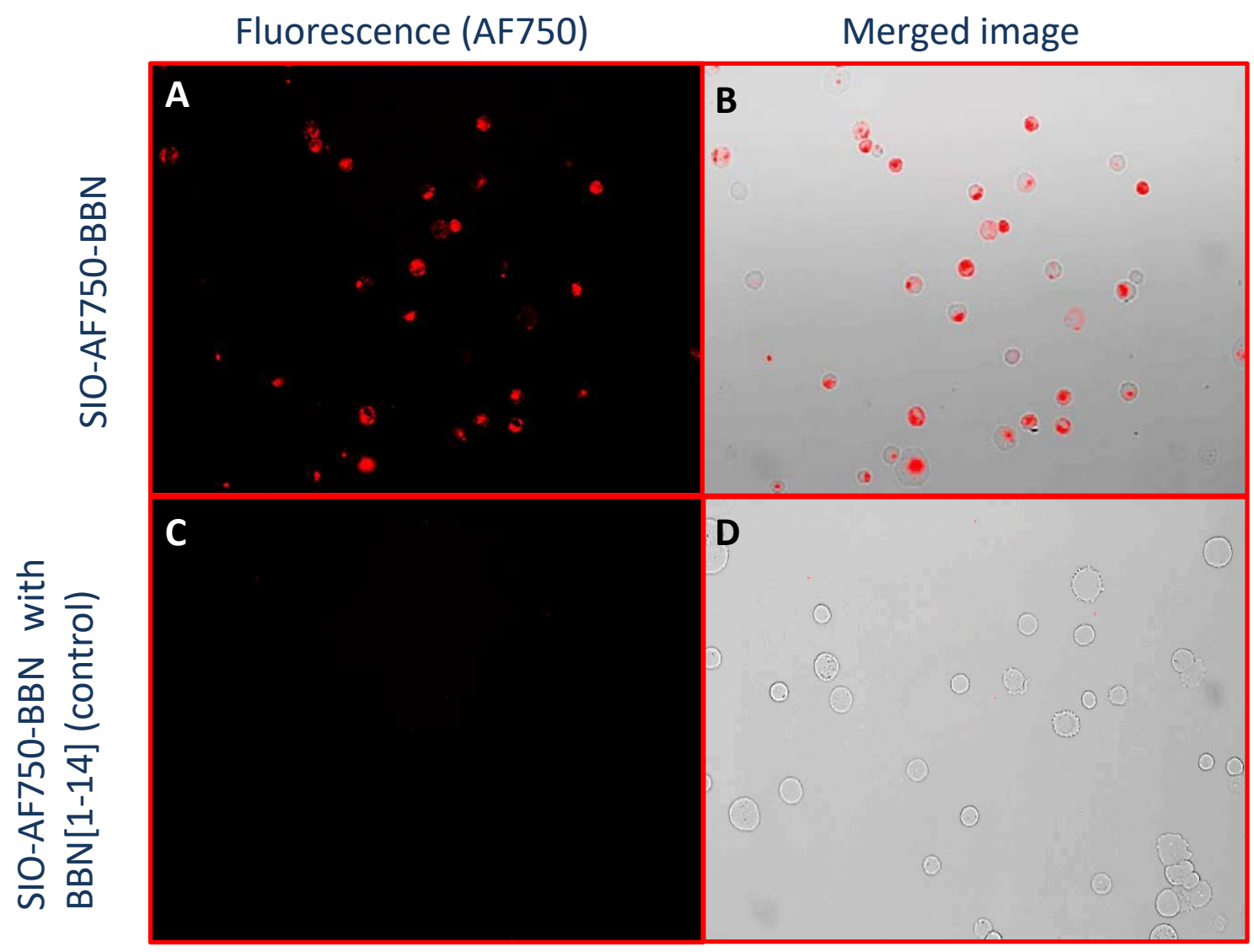

Figure 4.10 In vitro binding specificity of SIO-AF750-BBN to the GRP receptors on PC-3 cells. (A) NIR fluorescence image and (B) merged image (NIRF and bright-field) of SIOAF750-BBN in the uptake study in PC-3 cells; (C) NIR fluorescence image and (D) merged image (NIRF and bright-field) of SIO-AF750-BBN in the blocking study with the BBN[1-14] as the blocking agent in PC-3 cells

In vivo PA imaging of SIO-AF750-BBN in PC-3 tumor mouse model

In vivo photoacoustic imaging was performed pre- and 1-hour post-injection via tail vein for the uptake group and blocking group. As Figure $4.11 \mathrm{~A}$ shows, there is a significant PA contrast increase in tumor tissues in the uptake group than in the blocking group. To examine whether this PA enhancement is mainly due to the silica-coated 
nanoparticle or the AF750 dye, we also performed the PA imaging on mice injected with a small molecular probe AF750-Gpip-Sta-BBN, as shown in Figure 4.11B. Comparing the Figure 4.11 A and B, the PA enhancement from SIO-AF750-BBN is significantly (5-fold) higher than from AF750-Gpip-Sta-BBN at $750 \mathrm{~nm}$ excitation and throughout the full wavelength range. Qualitative ROI analysis (Figure 4.12) demonstrated that the SIOAF750-BBN PA enhancement (PA_post - PA_pre) is over 3-fold in the tumor tissues, and 1.5 -fold in the tumor surface vasculatures in the uptake group than in the blocking group. The SIO-AF750-BBN PA enhancement is also increased 5-fold than the small molecule AF750-Gpip-Sta-BBN group in the tumor tissues and the surface vasculatures. 

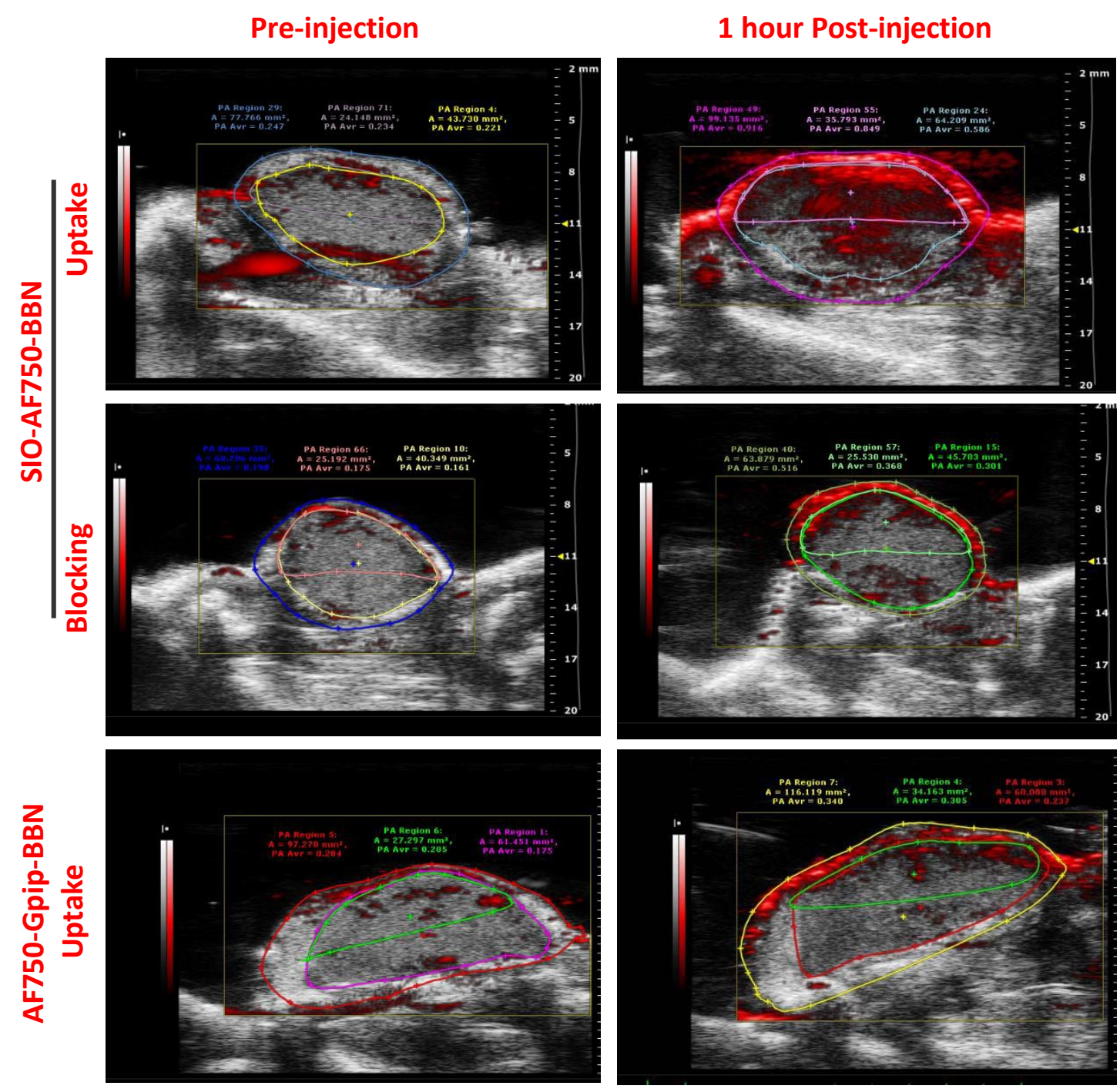

Figure 4.11 In vivo photoacoustic images (color scale) overlaid with ultrasound images (gray scale) pre- (left) and 1-hour post- (right) tail vein injection of SIO-AF750-BBN, uptake as compared to blocking with BBN[1-14], and AF750-Gpip-Sta-BBN, a small molecular BBN antagonist. The PA images shown are at $750 \mathrm{~nm}$ wavelength. Images were acquired using a VEVO 2100/VEVO LAZR system and an LZ250 PA transducer. 

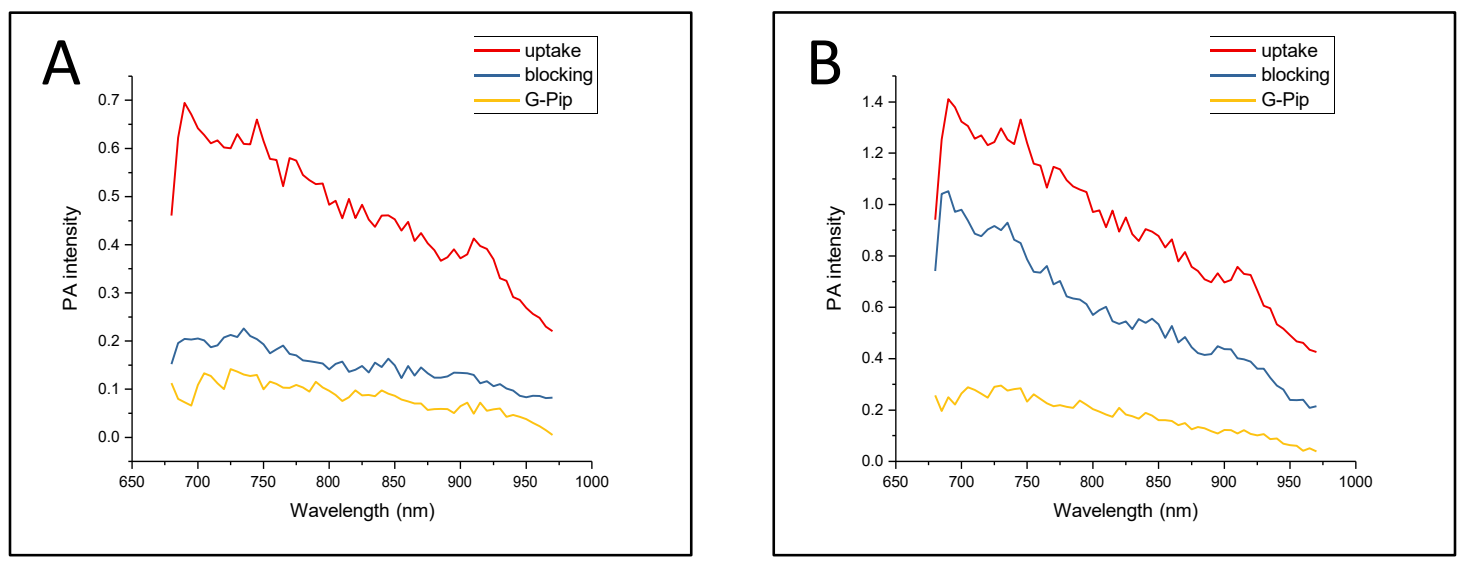

Figure 4.12 Photoacoustic intensity enhancements [PA_post - PA_pre] in PC-3 tumor tissues (A) and the tumor surface vasculatures (B) in the wavelength range of $680 \mathrm{~nm}$ to $970 \mathrm{~nm}$ acquired on VEVO LAZR system. The SIO-AF750-BBN PA enhancement is increased 3-fold than the blocking group in the tumor tissues and 1.5-fold in the tumor surface vasculatures. The SIO-AF750-BBN PA enhancement is also 5 times than the small molecular AF750-Gpip-Sta-BBN group in the tumor tissues and the surface vasculatures.

In vivo NIRF imaging and MRI evaluation of SIO-AF750-BBN. In the result of the in vivo IVIS study, the tumors of the mice in the uptake group displayed a significantly higher signal than that in the blocking group, as shown in Figure 4.13A. A kinetic biodistribution measurement was performed by measuring the epi-fluorescence intensities of tumors and muscles, and in the result, the tumor to muscle ratio for the uptake group was consistently higher than that for the blocking group, as shown in Figure 4.13B. 
Dosage: 93ug (Fe) SIO-AF750-BBN

Imaging time: 1 hour p.i.
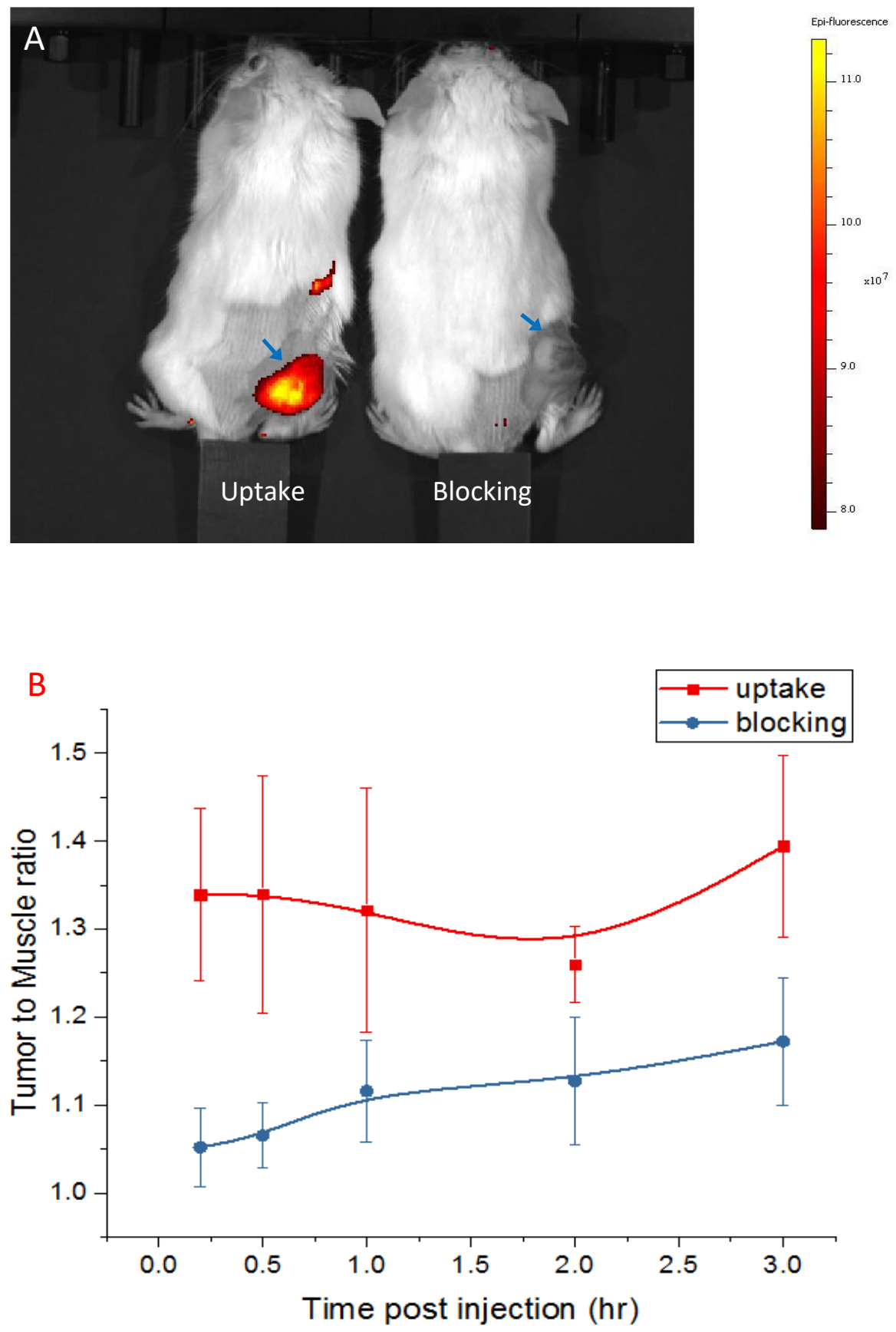

Figure 4.13 In vivo NIRF study of SIO-AF750-BBN in a prostate tumor mouse model. (A)The IVIS image of the mice from the uptake group and the blocking group at $1 \mathrm{hr}$ post tail vein injection. (B) In vivo tumor to muscle ratio versus different time points ( $n=3$ for each group). The TMR is significantly higher in the uptake group as compared to the blocking group at all the time points $(p<0.05)$. 
In the in vivo MRI study as displayed in Figure 4.14, there is no T2 relaxation enhancement observed in tumors and muscles in the uptake and blocking groups. In the T2-map (also called T2 relaxation parametric map) MRI images, the signals of the prostate tumor before and after the injection are not distinguishable, either. These results indicate that the i.v. administration of SIO-AF750-BBN at the current concentration cannot make an adequate enhancement on the tumors' contrast on the MRI.
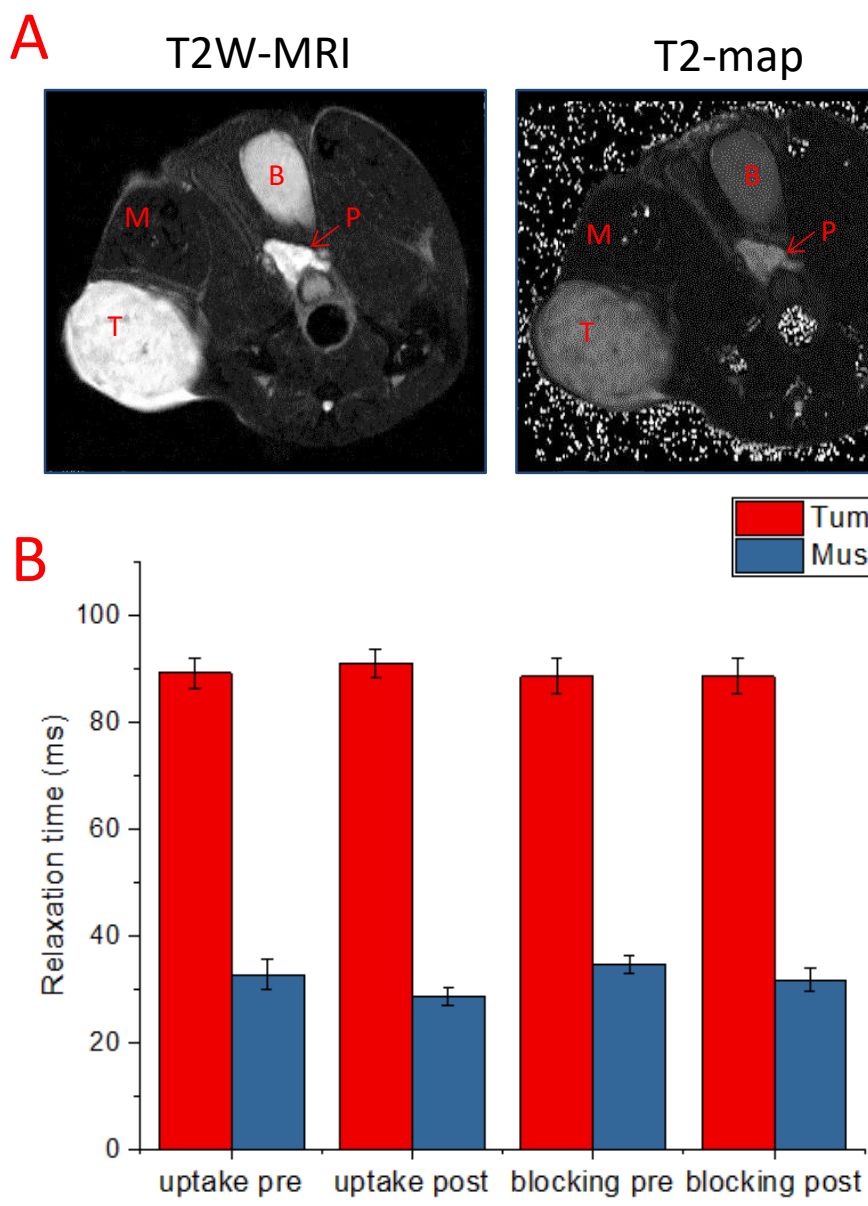

Figure 4.14 In vivo MRI study of SIO-AF750-BBN in SCID mouse model bearing PC-3 prostate cancer. (A) Representative MRI images: T2-weighted (T2W) MRI and T2-map of an axial slice across the tumor in a mouse 2 hours post injection of SIO-AF750-BBN. (B) 
The $\mathrm{T} 2$ relaxation time of tumor and muscle pre- and post-injection. There is no enhancement in the uptake group and the blocking group. T: Tumor, M: Muscle, B: bladder, and P: prostate.

Bio-distribution and histological studies. The mice were then sacrificed at $3.5-$ 4.5 hours post-injection, and organs were collected for ex vivo NIRF imaging study. As Figure 4.15 shows, liver and urine show the highest signal, indicating the excretion routes through the liver and renal/urine. The prostate tumor also shows higher fluorescence intensity in the uptake group than that in the blocking group. The pancreas is one of few normal organs that overexpress GRPr. In this result, the pancreas was observed to have higher fluorescent quantity in the uptake group than that in the blocking group, also validating the specific binding of the compound to GRPr. 

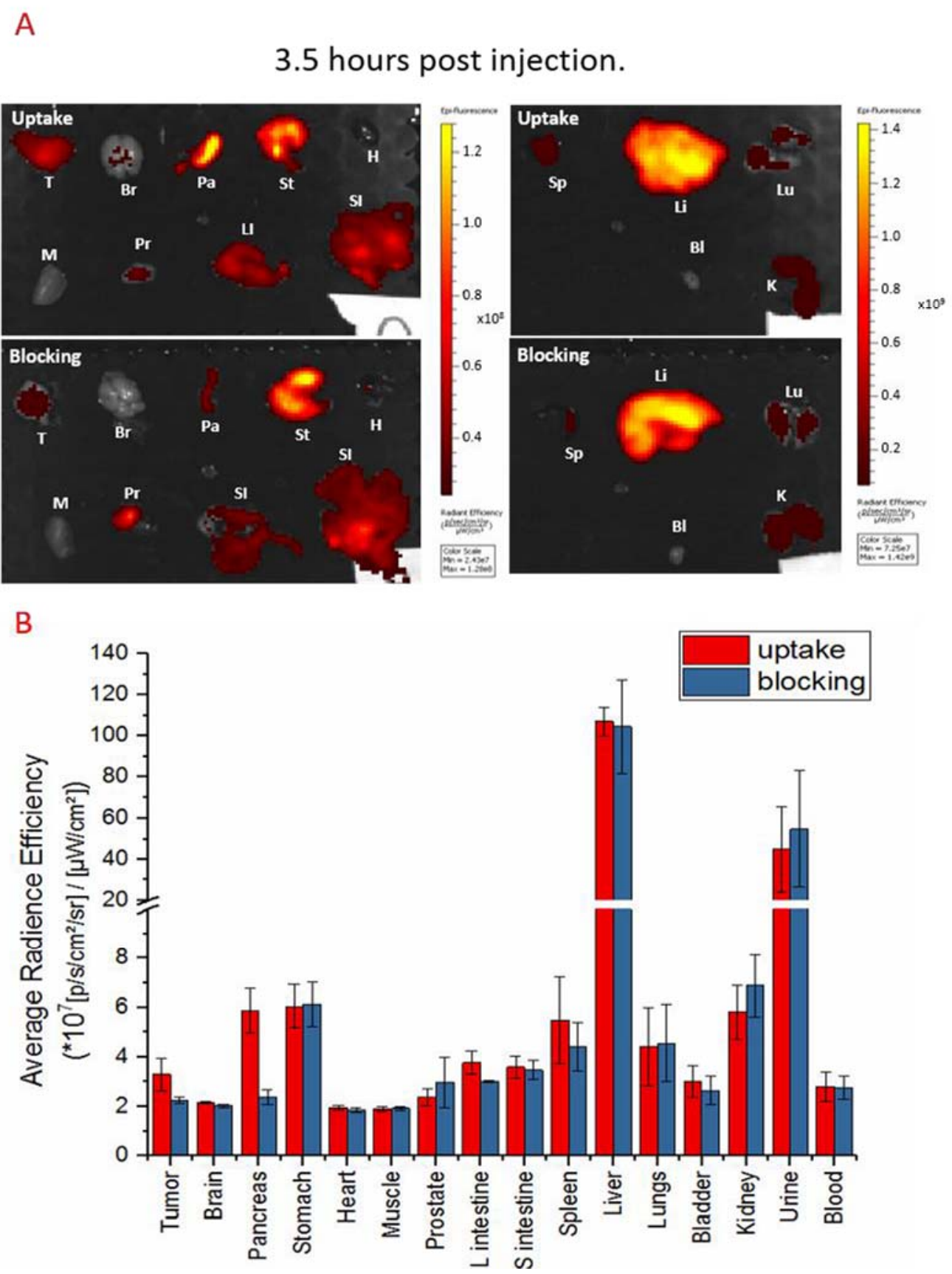

Figure 4.15 Ex vivo NIRF study of SIO-AF750-BBN with a prostate tumor mouse model. (A) The IVIS image of the organs from the uptake group as compared to the blocking group, letters denote: T: Tumor, Br: Brain, Pa: Pancreas, St: Stomach, H: Heart, M: Muscle, Pr: Prostate, LI: Large intestine, SI: Small intestine, Sp Spleen, Li Liver, Lu: Lungs, $\mathrm{BI}$ : Bladder, K: Kidneys. (B) Bio-distribution of SIO-AF750-BBN-AF750 in organs in the uptake groups as compared to the blocking group ( $n=3$ for each group). 
Finally, the tumors, pancreas, prostates and muscles from the ex vivo study were taken for a histological study, subjected to Prussian blue staining and nuclear red staining. As Figure 4.16 shows, in the tumor slices and pancreas slices in the uptake group, iron oxide nanoparticles that were stained to be blue, can be found in a large quantity. But in the other organs in the uptake group and the blocking group, the blue spots were hardly observed, validating the specific delivery of SIO-AF750-BBN to these organs that overexpress GPRr. 


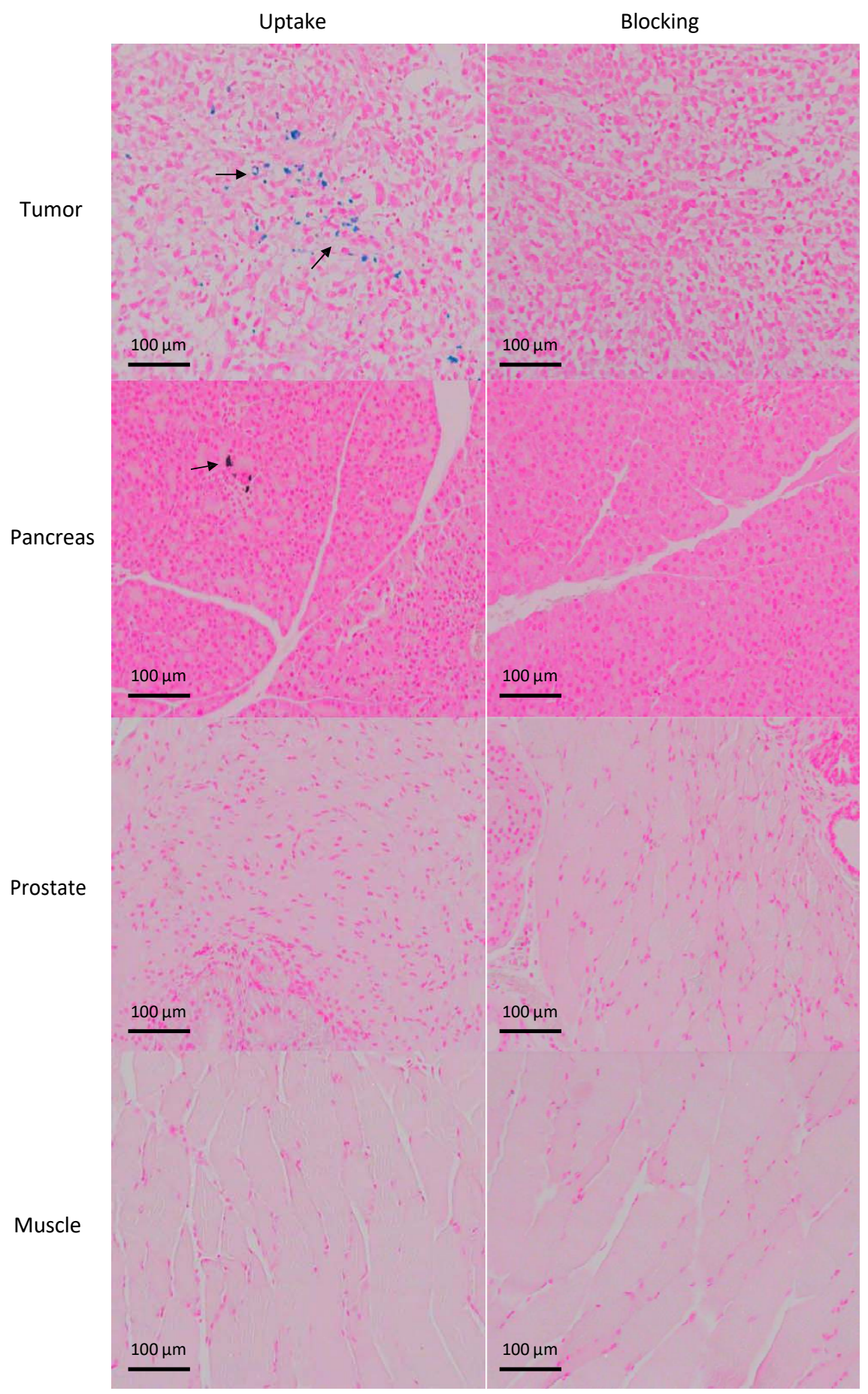

Figure 4.16 Images of histological slices regarding the SIO-AF750-BBN (Prussian blue staining) in tumors, pancreas, prostate, and muscles in the uptake group as compared to the blocking group. 


\section{Discussion}

Taking the outcomes of TEM, FTIR, pH measurements into consideration, the surface modification with silica for the $1 \mathrm{O}$ nanoparticle as well as the $\mathrm{COOH}$ functionalization with APTES-SA is successful. The purified nanoparticles display strong fluorescent intensity in the phantom study, indicating the successful conjugation with AF750-BBN that renders it a potential NIRF imaging probe with targeting ability. PA intensity of the SIO nanoparticle is enhanced by silica coating, compared to Feridex and USPIO(Cy7.5)-BBN. This enhancement is probably due to the change of interfacial heat conducting way from the IO nanoparticle to water due to silica layer. ${ }^{154}$ The SIO-AF750BBN has a high binding specificity to the GRPr on PC-3 cells as indicated by the uptake and blocking cellular experiments. Furthermore, we conducted animal studies in SCID mice bearing PC-3 prostate tumor xenografts to explore the in vivo binding affinity, specificity, and imaging enhancement ability on PC-3 tumors under multimodal imaging modalities, including PA detection, NIRF imaging, and MRI. Both in vivo PA imaging and NIRF imaging show a strong and highly specific enhancement of the tumors after intravenous injection of SIO-AF750-BBN. The MRI imaging, however, did not display any significant enhancement of the tumors. MRI is an imaging modality that lacks sensitivity, and the quantity of the compound in the tumors is not sufficiently high to make an adequate difference to elevate the contrast visually. The mice injected with the compounds did not show any abnormal behavior, indicating no severe toxicity for this compound. 
SIO-AF750-BBN demonstrated a very high PA contrast enhancement (PA_post PA_pre) in the tumor tissues, with a 3-fold increase in the uptake group as compared to the blocking group, and a 5-fold increase than the small molecule AF750-Gpip-Sta-BBN group.

The ex vivo NIRF bio-distribution study also demonstrated the specific binding of the compounds to the PC-3 tumors and pancreas that overexpress GRPr. The histological study, where iron oxide nanoparticle content was found in the tumor and pancreas in the uptake group, but not in the blocking group, validated that the SIOAF750-BBN nanoparticles were delivered into the tumors in the uptake group. Urine and liver show the strongest NIRF signals at the sacrifice at $3.5-4.5$ hours post-injection, implementing a renal-urine excretion route for a fairly large portion of the nanoparticles. From the TEM observation, there exists single nanoparticle, dimer, trimer or bigger aggregations of the nanoparticles in the solution. The single nanoparticle and dimers are likely quickly removed through kidneys due to their small dimension, and the bigger particles are usually captured by the macrophages in the liver and spleen and being excreted for a relatively longer time. No significant amount of fluorescence was detected in the blood in the ex vivo result at 3.5 - 4.5 hours post injection, indicating a fast clearance of the compound in the blood circulation. It's probably because the compound has a negatively charged surface contributed by $\mathrm{Si}^{-} \mathrm{O}^{-}$and $\mathrm{COO}^{-}$that could potentially pose a nonspecific protein adsorption from plasma and serum in the blood. The problem may be solved through reversibly switchable polymer with 
cationic/zwitterionic/anionic behavior through synergistic protonation and deprotonation on the surface. More studies need to be conducted to address more details about the pharmacokinetics, biodistribution, excretion route and long term toxicity.

The future work will include an in vitro study where we will capture circulating cancer cells from cancer patients to mix with blood cells and incubate with this hybrid nanoparticle for the photoacoustic flow cytometry (PAFC) test to demonstrate the potential of highlighting CTCs from a background of blood cells. To obtain cancer patients' blood sample, we will need the collaboration with Dr. Richard Hammer in MU clinics. SIO-AF750-BBN would be incubated with the blood sample, followed by washing away free compounds not loaded by cells, and a control group may also be taken with BBN[1-14]. PA and NIRF imaging would be performed to display nanoparticles' loading on the CTCs for the uptake group and the control group. In Wang et al.'s work, he successfully visualized clusters of CTCs from the red and white blood cells in patients' blood samples by hematoxylin staining. ${ }^{155}$ There are at least two advantages of the SIOAF750-BBN nanoparticle developed in this work. First, SIO-AF750-BBN is highly specific to the cancer cells overexpressing GRP receptors, making an exclusive PA signal enhancement of cancer cell and CTC. Second, the PA signal enhancement is $325 \%$ at $750 \mathrm{~nm}$ wavelength and $250 \%$ throughout the wavelength range in the tumor tissues, likely due to the coupling of both silica-coating on the IO nanoparticle and the NIRF dye on the surface, thus potentially improve the PA detection sensitivity of rare CTCs. 
Finally, we expect to have a chance to perform an in vivo test for a direct CTC detection on the xenograft prostate cancer model with a photoacoustic flow cytometry. Mice ear is thin (250-300 um) and optically semi-transparent. It has well-distinguished veins and arteries that locate only 30-100um deep with a diameter of about 50um, which is desirable for PAFC measurement. ${ }^{133}$ The challenge is the sensitivity of detection, which is determined by the binding efficacy of nanoparticles and the instrumental sensitivity of PAFC. To break the limit of the detection sensitivity, an external magnet may be used to concentrate nanoparticles loaded CTCs in the focused areas. In collaboration with the research group led by Dr. Ping Yu, one PAFC instrument will be constructed for the in vivo CTCs detection, whose outcome will be of great clinical significance. This experiment will be of great clinical significance to initiate instant CTC detection for improvements of early cancer detection.

\section{Acknowledgement}

I would like to thank Fengfei Wang's help in FTIR sample preparation and measurements. MU Electron Microscopy (EM) Core Facility's support in TEM practice, MU Molecular Cytology Core's support in fluorescent microscope practice, Terry Carmack's help in PA imaging and organ collection, Li Lee and Ran Li's assistance in MRI and NIRF imaging, and Lisa Watkinson for tumor inoculations. 


\section{Chapter V. Near-Infrared Fluorescence Dye Labeled Bombesin Antagonist Peptides for Molecular Imaging of GRPR: an In Vitro and In Vivo Evaluation in Human Prostate Cancer}

\section{Introduction}

Prostate cancer is the second leading cause of cancer-related mortality in the United States for men. ${ }^{97}$ Prostate cancer can be screened or early-diagnosed with prostate-specific antigen test (PSA test), digital rectal examination (DRE) and biopsy examination, but none of them is accurate enough, and overdiagnosis and misdiagnosis frequently occur. ${ }^{98,99} \mathrm{To}$ overcome these problems or limitations, molecular imaging, especially when applied with a tumor-specific targeting probe has shown a good promise. ${ }^{70}$

Bombesin (BBN) that was initially isolated from the skin of a fire-bellied toad, ${ }^{100}$ has attracted considerable interests due to its ability to target to gastrin releasing peptide receptor (GRPr or BB2r) overexpressed by tumors. BBN closely resembles two mammalian BBN-related peptides: gastrin-releasing peptide (GRP) and neuromedin (NMB).GRP and BBN have the same seven amino acids [-Trp-Ala-Val-Gly-His-Leu-Met$\mathrm{NH} 2$ ] in their C-terminus that govern their agonist activity. ${ }^{101,102}$ The BBN receptor family consists of four subtypes: (1) neuromedin B receptor (NMB-r or BB1r), (2) gastrin- 
releasing peptide receptor (GRPr or $\mathrm{BB} 2 \mathrm{r}$ ), (3) the orphan receptor (BB3r) and (4) the amphibian receptor (BB4r), and it has been demonstrated that BB2 receptors are overexpressed in various tumor types, including prostate, pancreatic, gastric and small cell lung cancers. ${ }^{102-108}$ The BBN analogs have high binding affinities to GRPr, and therefore it could serve as targeting vector for tumor imaging. ${ }^{109,110}$ In fact, much work has been done on developing radiolabeled, or fluorescence dye labeled bombesin analogs with positive outcomes in the past decades. $68,69,72,81,90,111-121$

Several years ago, bombesin antagonists that are poorly internalized into cancer cells were discovered to have equally high binding affinities, desirable pharmacokinetics, and biodistribution, compared to bombesin agonists, for GRPr overexpressed prostate cancer cells. ${ }^{7}$ Additionally, bombesin antagonists have the potential to avoid side effects that could be induced by bombesin agonist, including abdominal cramps, nausea, etc. BBN agonists are known to stimulate tumor growth and angiogenesis, while BBN antagonists may prevent this side effect as well. ${ }^{9,10}$ Very recently, some efforts were being devoted to exploring the potential of bombesin antagonist as a novel targeting vector, and a lot of promising outcomes were achieved. ${ }^{70,116,122-125}$

In this work, we would investigate the potential of D-Phe-GIn-Trp-Ala-Val-GlyHis-Sta-Leu-NH2 (Sta-BBN), an antagonist analog of BBN peptide, as the targeting vector with a very high sensitivity, specificity and imaging efficacy for near infrared fluorescence (NIRF) imaging of GRPr positive cancer such as prostate cancer. 
Near infrared fluorescence imaging is a good alternative to nuclear imaging for prostate cancer through monitoring prognosis and tumor progression at a low cost and short operation time. ${ }^{111}$ Fluorescence dyes that emit optical signals at a wavelength of $600 \mathrm{~nm}$ to $900 \mathrm{~nm}$ are beneficial for achieving higher penetration depth since water, lipids, and hemoglobins exhibit minimum absorption and light scattering in this wavelength range. ${ }^{56,85,126}$ Within this wavelength range, three fluorescence dyes are selected and studied, including Dylight800/Dylight800-4PEG (excite/emission: 770/794nm), Alexa Fluor750 (749/775nm) and Cyanine7.5 (788/808nm). In addition to the peptide and the dye, the linker that connects them will also have a profound influence on probe's binding selectivity, affinity, pharmacokinetics, and biodistribution. ${ }^{117,127,128}$ Three different linkers, Gly-Ser-Gly (GSG) as a long flexible linker, ${ }^{127}$ Gly-4Amino_Carboxymethyl_Piperidine (G-Pip) as a positively charged linker, ${ }^{128}$ and 6-(amino)hexanoic acid $-\mathrm{NH}\left(\mathrm{CH}_{2}\right)_{5} \mathrm{COOH}$ (6Ahx) as a hydrophobic linker, are selected to conjugate to the $\mathrm{N}$-terminus of the bombesin antagonist (Sta-BBN).

In this work, a total of nine NIRF dye labeled bombesin antagonist with different choices of linkers (G-Pip, GSG, and 6Ahx) and organic fluorescent dyes (AF750, DL800/DL800-PEG and Cy7.5) would be synthesized to explore the possibility of specifically enhancing prostate tumor's contrast in NIRF imaging systems. Analytical chemistry methods are used to characterize all the compounds. In vitro and in vivo experiments are designed to explore the compounds' binding selectivity, binding affinity to prostate cancer cells, as well as the pharmacokinetics and bio-distributions in a 
mouse model bearing prostate tumor xenografts. The outcomes will be analyzed to screen out the optimal agent for the future studies.

\section{Materials}

Gly-Ser-Gly-DPhe-Gln-Trp-Ala-Val-Gly-His-Sta-Leu-NH2 (GSG-Sta-BBN), Gly-GPip_Carboxymethyl_Piperidine-DPhe-GIn-Trp-Ala-Val-Gly-His-Sta-Leu-NH2 (G-Pip-Sta$\mathrm{BBN})$ and $\left[\mathrm{NH}_{2}\left(\mathrm{CH}_{2}\right)_{5} \mathrm{CO}\right]-$ DPhe-Gln-Trp-Ala-Val-Gly-His-Sta-Leu-NH2 (6Ahx-Sta-BBN) were synthesized by a commercial source (CPC Scientific, Sunnyvale, CA) using conventional solid phase peptide synthesis (SPPS) method. Cyanine 7.5 NHS ester was purchased from Lumiprobe (Hallandale Beach FL). DyLight800/Dylight800-4PEG NHS ester and Alexa Fluor ${ }^{\circledR} 750$ NHS ester were purchased from ThermoFisher Scientific (Waltham, MA). $\mathrm{NaHCO}_{3}, \mathrm{DMF}$, and other regular reagents were purchased from SigmaAldrich (St. Louis, MO). Bombesin[1-14] from American peptide company (Sunnyvale, CA) was used as the blocking agent in this study. ${ }^{125}$-Tyr ${ }^{4}-B B N$ from PerkinElmer (Waltham, MA) was applied for the half maximal inhibitory concentration (IC50) determination. Fluo-4NW calcium assay kit from Thermo Fisher Scientific (Waltham, MA) was used for the calcium mobilization study. 


\section{Methods}

\section{Chemical Conjugation, Purification and Mass Spectrum Examination.}

To 2 mg peptide (GSG-Sta-BBN or G-Pip-Sta-BBN or 6Ahx-Sta-BBN) that dissolved in $150 \mu \mathrm{l} 0.1 \mathrm{M} \mathrm{NaHCO} 3$ buffer $(\mathrm{pH}=8.4), 1.06 \mathrm{mg}$ AF750 in $53 \mu \mathrm{l}$ DMF was added dropwise. The reaction was stirred at $4^{\circ} \mathrm{C}$ overnight. The procedure was protected from light. Similarly, DL800 and Cy7.5 conjugated bombesin antagonist were made. For DL800 conjugated bombesin antagonist, $1 \mathrm{mg}$ peptide (GSG-Sta-BBN or G-Pip-Sta-BBN or 6AhxSta-BBN) in $30 \mu \mathrm{l} 0.1 \mathrm{M} \mathrm{NaHCO} 3(\mathrm{pH}=8.5)$ buffer with $20 \mu \mathrm{l}$ DMF was added into $0.32 \mathrm{mg}$ DL800/DL800-PEG in $32 \mu \mathrm{L}$ DMF. The reaction was stirred at $4^{\circ} \mathrm{C}$ in the dark overnight. For Cy7.5 conjugated bombesin antagonist, $0.754 \mathrm{mg}$ peptide (GSG-Sta-BBN or G-PipSta-BBN or 6Ahx-Sta-BBN) in $94.25 \mu \mathrm{l} 0.1 \mathrm{M} \mathrm{NaHCO}$ buffer(pH=8.5) with $18.85 \mu \mathrm{l}$ DMF was added into $0.3 \mathrm{mg}$ Cy7.5 in $60 \mu \mathrm{DMF}$, and the reaction was stirred at $4^{\circ} \mathrm{C}$ in the dark overnight. All products were purified with a Shimadzu reverse phase highperformance liquid chromatography (RP-HPLC) (Kyoto, Japan) and a Phenomenex $5 \mu \mathrm{m}$ C18 $300 \AA 250 \times 4.6$ mm column, and were subsequently examined on 4700 MALDI TOF/TOF mass spectrometer (Applied Biosystem Inc., now AB Sciex). The absorption spectrum of The AF750-G-Pip-Sta-BBN was measured on Shimadzu UV-Vis Spectrometer

UV-2401PC (Kyoto, Japan), and the fluorescence spectrum was measured on a Shimadzu Spectrofluorophotometer RF-6301 (Kyoto, Japan) with excitation at 692nm. 


\section{Cell Culture}

The human prostate cancer (PC-3) cell-line was supplied by the Cell and Immunobiology Core Facility at University of Missouri. All PC-3 cells were cultured in medium consisted of 89\% RPMI 1640 from Thermo Fisher Scientific (Waltham, MA), 10\% fetal bovine serum (FBS) from Sigma-Aldrich (St. Louis, MO) and $1 \%$ penicillin from Invitrogen (Carlsbad, CA). Trypsin from Invitrogen (Carlsbad, CA), containing $0.25 \%$ EDTA, was used for suspending cells. Cells were cultured in the growing environment $\left(37^{\circ} \mathrm{C}\right.$ and $\left.5 \% \mathrm{CO}_{2}\right)$ using a Forma water-jacketed incubator from Fisher Scientific (Waltham, MA).

\section{Cellular Uptake, Blocking and Internalization Assay}

PC-3 cells were suspended by trypsin and washed with culture medium. $1 \mathrm{ml}$ cell suspension, containing 4 million PC-3 cells, was placed into each tube. In the specific binding assay, namely, uptake test, $1 \mathrm{ml}$ cell suspension was incubated with $0.14 \mathrm{nmol}$ NIRF dye labeled bombesin antagonist for $45 \mathrm{~min}$, washed with ice-cold culture medium and ice-cold PBS 3 times before the observation. In the blocking assay, $1 \mathrm{ml}$ cell suspension was pre-incubated with $56.8 \mathrm{nmol} B B N[1-14]$ for $10 \mathrm{~min}$ and then incubated with $0.14 \mathrm{nmol}$ NIRF dye labeled bombesin antagonist for $45 \mathrm{~min}$, then washed with ice-cold culture medium and ice-cold PBS 3 times for the following observation. In the internalization assay, $1 \mathrm{ml}$ cell suspension was incubated with $0.14 \mathrm{nmol}$ NIRF dye labeled bombesin antagonist for $45 \mathrm{~min}$ and subsequently washed with an ice-cold 
$\mathrm{pH}=2.5$ buffer $(0.2 \mathrm{M}$ acetic acid, $0.5 \mathrm{M} \mathrm{NaCl})$ for two times. At last, it was washed with ice-cold PBS before the observation. All the incubation processes were taken at $37^{\circ} \mathrm{C}$ and $5 \% \mathrm{CO}_{2}$ in the dark. After the wash cycles, concentrated cell suspension samples were examined for microscopic NIRF signals in the cells. Briefly, $10 \mu \mathrm{l}$ of the concentrated cell suspension was dropped onto a glass slide and covered using a coverslip. The microscopic imaging was performed on a Zeiss Axiovert 200M inverted microscope (Gottingen, Germany) installed with a Cy7 filter set at the excitation wavelength range of $673-748 \mathrm{~nm}$ and emission wavelength range of $765-835 \mathrm{~nm}$.

\section{Calcium Mobilization Study to PC-3 Cells.}

Intracellular calcium mobilization was measured in PC-3 cells using the Fluo-4NW calcium assay kit according to the method described by Cescato et al ${ }^{7} .40,000$ PC-3 cells in $200 \mu \mathrm{l} \mathrm{RPMI}$ 1640(10\% FBS and 1\% Penicillin) were seeded in the 96 well plate for one day. The RPMI culture media were then removed from the seeded cells, followed by the addition of $100 \mu \mathrm{l}$ Fluo-4NW dye in the assay buffer (2\%V of $1 \mathrm{M}$ HEPES in $1 \mathrm{X}$ HBSS solution) with $2.5 \mathrm{mM}$ probenecid to each well for an incubation for $30 \mathrm{~min}\left(37^{\circ} \mathrm{C}, 5 \%\right.$ $\mathrm{CO}_{2}$ ) and another $30 \mathrm{~min}$ at room temperature. The fluorescence signal intensity of each well in the presence of compounds at different concentrations was measured in a kinetic experiment for $60 \mathrm{~s}$ at room temperature by a Synergy H4 hybrid reader (Biotek, Winooski, VT) at the excitation of $485 \mathrm{~nm}$ and the emission of $525 \mathrm{~nm}$. For a baseline measurement, one well of cells without any treatment by the Fluo-4NW dye was 
included. BBN[1-14], as a bombesin agonist, was also measured and used as a reference curve to the NIRF dye labeled bombesin antagonists. Each data point was triplicated, and data were fitted to obtain the calcium mobilization curves.

\section{Binding Affinity to PC-3 Cells.}

In vitro competitive cell binding assays of the compounds were performed against the gold standard ${ }^{125} \mathrm{I}-\mathrm{Ty}^{4}-\mathrm{BBN}$ for the BB2 receptor. The quantification of binding affinity was represented by the inhibitory concentration fifty percent, IC50. Briefly, $3 \times 10^{4}$ PC-3 human prostate cancer cells in the culture medium were incubated with $30,000 \mathrm{cpm}^{125} \mathrm{I}-\mathrm{Tyr}^{4}-\mathrm{BBN}$ and increasing concentrations of the compound (AF750G-Pip-Sta-BBN or AF750-GSG-Sta-BBN) at $37^{\circ} \mathrm{C}$ and $5 \% \mathrm{CO}_{2}$ for $45 \mathrm{~min}$. The incubation medium was subsequently aspirated, and the cells were washed three times with icecold media. Cell-associated radioactivity was determined by counting in a Wizard 3" 1480 automatic gamma counter (PerkinElmer, Waltham, MA). This procedure was repeated for three times for statistical significance.

\section{Mouse Model}

All the animal studies were conducted in line with the highest standards of care outlined in the National Institutes of Health Guide for care and use of laboratory animals, as well as the policy and handling procedures for animal research in the Harry 
S. Truman Memorial Veterans Hospital. The in vivo studies were performed on SCID (severely compromised immunodeficient) mice (The Jackson Laboratory, male, 20-25 g body weight, 4-6 weeks age) bearing human PC-3 prostate cancer xenografts. Four weeks before the experiment, the mice were subjected to inoculations of PC-3 cells. In the preparation, 3 million PC-3 cells were washed with PBS three times to remove the culture medium. Matri-gel was taken out from storage at $-80^{\circ} \mathrm{C}$ and placed into ice for melting. $1 \mathrm{ml}$ melted matri-gel was mixed with $2 \mathrm{ml}$ PBS added to the tube that contains PC-3 cells. In the inoculation, the mouse was anesthetized, and the inferior back fur that is close to the lateral flanks was shaved off. Afterwards, 3 million PC-3 cells in $0.1 \mathrm{ml}$ matri-gel were injected into the right flank. The mice were then housed in sterile microisolator cages with four in each one and they were daily fed with autoclaved rodent chow (Ralston Purina Company, St. Louis, MO) and water ad libitum. CF1 mice (Charles River Laboratory, male, 25-35 g, 8-12 weeks age) were also used for dosage determination.

\section{Dosage Determination for In vivo Study}

In the first stage, the desirable dosage was explored for in vivo imaging. Mice in the uptake group of AF750-G-Pip-Sta-BBN were injected with $39.1 \mathrm{nmol}, 27.6 \mathrm{nmol}$, and $19.2 \mathrm{nmol}$ respectively via the tail vein. In the blocking group of AF750-G-Pip-Sta-BBN, two mice were co-injected with $27.6 \mathrm{nmol}$ and $19.2 \mathrm{nmol}$ AF750-G-Pip-Sta-BBN and $123.5 \mathrm{nmol}$ unlabeled BBN[1-14] as the blocking agent. For AF750-GSG-Sta-BBN, two 
mice in the uptake group were injected with $16.0 \mathrm{nmol}$, and $11.0 \mathrm{nmol}$ AF750-GSG-StaBBN and two mice in the blocking group was co-injected with 16.0 and $11.0 \mathrm{nmol}$ AF750-GSG-Sta-BBN and 123.5 and $77.8 \mathrm{nmol}$ BBN[1-14], respectively. DL800-6Ahx-StaBBN and Cy7.5-6Ahx-Sta-BBN were also preliminarily tested in mice at appropriate doses for uptake and blocking studies to explore the possible differences made by different fluorescent dyes.

\section{In vivo NIRF and MRI Imaging}

Since the number of mice is limited, the number per each group is not strictly the same. A total of twenty mice bearing human PC-3 tumor xenografts were examined. Among them, eleven mice were used in the study at the desired dosage and assigned as the follows: two in the AF750-G-Pip-Sta-BBN uptake group and one in the blocking group. One was used as the AF750-GSG-Sta-BBN uptake group and one as the blocking group. Three were used for the AF750-6Ahx-Sta-BBN uptake group and three for the blocking group. For AF750-G-Pip-Sta-BBN and AF750-6Ahx-Sta-BBN studies, mice in the uptake group were intravenously injected with $0.44 \mathrm{nmol}$ of the compound, and mice in the blocking group were pre-injected with $38.9 \mathrm{nmol} B B N[1-14]$ followed by an injection of $0.44 \mathrm{nmol}$ AF750-G-Pip-Sta-BBN. For AF750-GSG-Sta-BBN studies, the uptake group was injected with $2.19 \mathrm{nmol}$ of the compound, while the blocking group was preinjected with $58.4 \mathrm{nmol}$ BBN[1-14] followed by an injection of $2.19 \mathrm{nmol}$ AF750-GSGSta-BBN. 
After the intravenous injections via the tail vein, all mice were subjected to NIRF imaging at $15 \mathrm{~min}, 30 \mathrm{~min}, 1 \mathrm{hr}, 2 \mathrm{hr}$ (some will be imaged at $3 \mathrm{hr}$ ) and finally being sacrificed for acquiring bio-distribution data for an ex vivo analysis. All the in vivo and ex vivo NIRF imaging studies were conducted on an IVIS spectrum imaging system from PerkinElmer (Waltham, MA) equipped with a cooled charge-coupled device (CCD) camera and a 150-W quartz halogen light source. The mice were anesthetized under 1.5 $-3.5 \%$ isoflurane in oxygen via nose cones and maintained a body temperature of $37^{\circ} \mathrm{C}$ during the imaging. Data acquisition was taken at a specific filter combination of excitation at $745 \mathrm{~nm}$ and emission at $800 \mathrm{~nm}$. Other parameters were set as: exposure time at $1 \mathrm{sec}, \mathrm{f} / \mathrm{stop}$ at 2 , the binning number at 8 , lamp at high/low level and field of view at $13.2 \mathrm{~cm}$. Drawing of the region of interest (ROI) and semi-quantification of average fluorescence intensity were performed using Living Image 4.4. The tumor to muscle ratio (TMR) was measured by dividing average radiant efficiency $\left([\mathrm{p} / \mathrm{sec} / \mathrm{cm} 2 / \mathrm{sr}] /\left(\mu \mathrm{W} / \mathrm{cm}^{2}\right)\right)$ of the tumor by the counterpart of muscle.

In addition to NIRF study, MRI images were taken on these mice prior to the injection to obtain the anatomical structure of tumor and other organs for correlation with the NIRF images. Mice were anesthetized under $1.5-2.5 \%$ isoflurane in oxygen via a nose cone and maintained the body temperature with warm air. T2-weighted (T2W) MRI axial scans were performed on a $7 T / 20 \mathrm{~cm}$ Bruker AVANCE III BioSpec system (Bruker BioSpin, Belarica, MA) equipped with a Quadruture-35 $\mathrm{mm}$ radiofrequency (RF) coil with the parameters: time of repetition (TR) at $2500-3500 \mathrm{~ms}$, time of echo (TE) at 
30 or $45 \mathrm{~ms}, 13-19$ slices, slice thickness of $1 \mathrm{~mm}$, matrix at $256 \times 128$, field of view at $3.00 \times 2.50 \mathrm{~cm}$.

\section{Histological Study}

The tumors, muscles and normal prostates of the mice used in the in vivo study were collected and preserved in $4 \%$ formalin and transferred to $70 \%$ ethanol, and subsequently embedded in paraffin blocks and cut to $5 \mu \mathrm{m}$ thick slices on a microtome. They were stained with DAPI, and the microscopic imaging was taken under a Carl Zeiss Axiovert 200M inverted microscope equipped with a Cy7 filter (excitation wavelength ranges $673-748 \mathrm{~nm}$, and emission wavelength ranges $765-835 \mathrm{~nm})$.

\section{Statistical analysis}

Quantitative data were expressed as the mean \pm SD. Means were compared by analysis of a student's $t$-test. $P$ values of less than 0.05 were considered to be statistically significant. 


\section{Result}

\section{Synthesis and Purification}

The amino acid sequence -DPhe-GIn-Trp-Ala-Val-Gly-His-Sta-Leu-NH2 (Sta-BBN) was selected as a bombesin antagonist sequence. Three linkers, -Gly4Amino_Carboxymethyl_Piperidine- (G-pip), -Gly-Ser-Gly- (GSG), and - $\mathrm{NH}\left(\mathrm{CH}_{2}\right)_{5} \mathrm{CO}$ (6Ahx), were chosen with a various hydrophobicity and charge as the pharmacokinetics modifiers. All three synthesized peptides, G-pip-Sta-BBN, GSG-Sta-BBN and 6Ahx-StaBBN, are water-soluble, have a HPLC retention time of $12.0 \mathrm{~min}(27 \%-37 \%$ B buffer in 20 min, flow rate $1 \mathrm{ml} / \mathrm{min}$. A: $0.1 \%$ TFA in $\mathrm{H}_{2} \mathrm{O}$, B: $0.09 \%$ TFA in $80 \% \mathrm{ACN}+20 \% \mathrm{H}_{2} \mathrm{O}$ ), 8.8 $\min (32 \%-42 \%$ B buffer in $20 \mathrm{~min})$, and $8.7 \mathrm{~min}(33 \%-43 \% \mathrm{~B}$ buffer in $20 \mathrm{~min})$, and a molecular weight (MW) of 1310.6Da, 1314.5Da, and 1226.5Da, respectively. Three NIRF NHS ester dyes, Alexa Fluor ${ }^{\circledR} 750$ (AF750), DyLight800 (DL800), and (Cy7.5), were investigated in the conjugation reactions. A total of nine BBN antagonist analogs were synthesized as illustrated in Figure 5.1. The reactions worked well without any precipitations generated in the process. The products were purified by RP-HPLC with a pre-set gradient (the percentage of $B$ solvent in the $A$ and $B$ solvent mixture during the mobile phase: $0-15 \min : 20 \%-40 \%, 15-20 \min : 40 \%-80 \%, 20-25 \min : 80 \%-20 \%$, where A solvent represents $0.1 \%$ TFA in water and $B$ solvent represents $0.1 \%$ TFA in acetonitrile). 


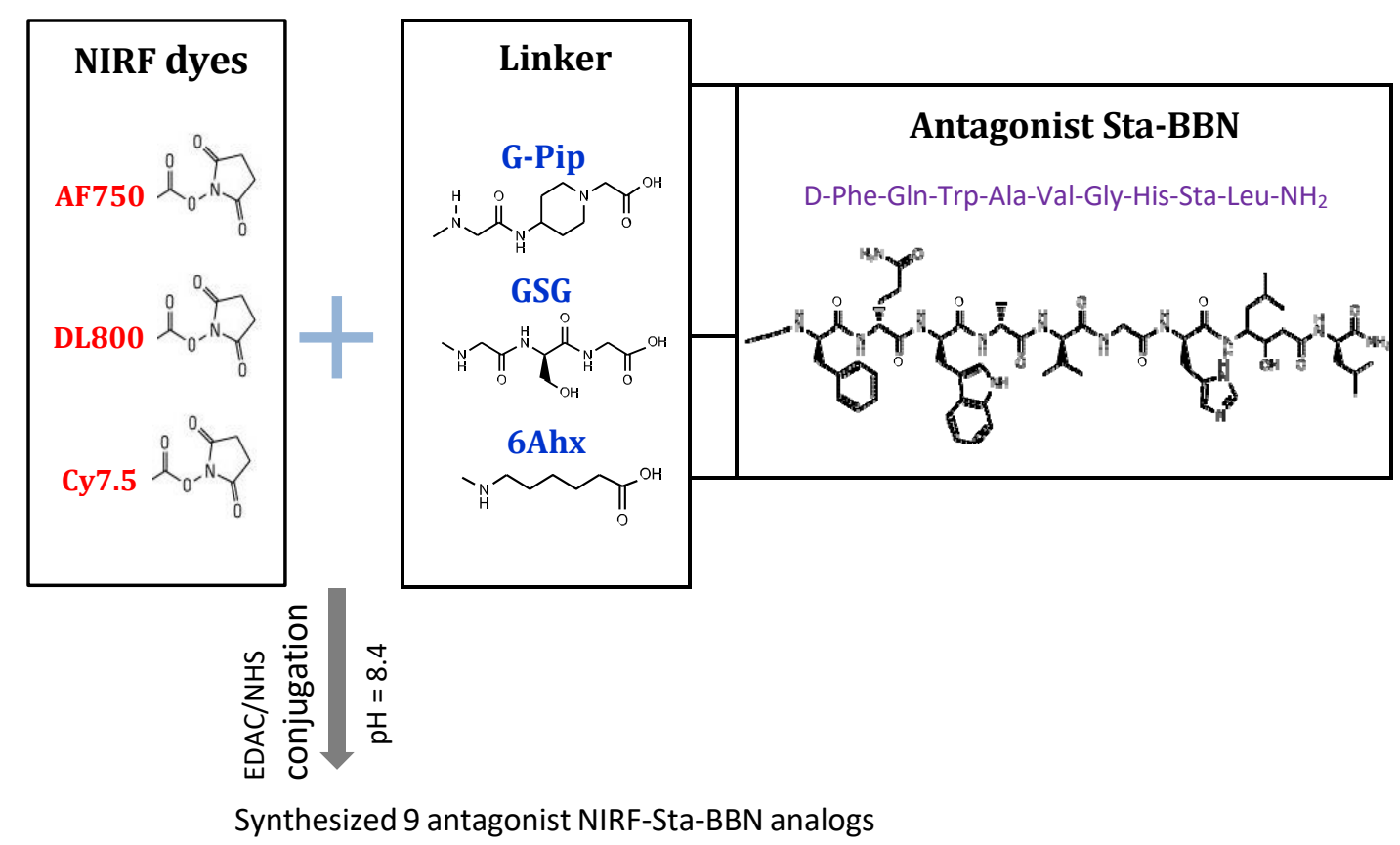

Figure 5.1 Scheme of synthesis of bombesin antagonist with NIRF dyes. (B) The fluorescence profile for AF750-G-Pip-Sta-BBN

The characteristics of the compounds are summarized in Table 4.1. All the compounds were purified with purity higher than $95 \%$. The compounds collected were analyzed for a mass spectrum, whose values match the values calculated based on the chemical formula for all the compounds, validating the success of the chemical synthesis. As shown in Table 4.1, AF750 conjugates show good water solubility, high yield, and easy HPLC purification, DL800 conjugates show good water solubility, hard HPLC separation, and an average yield, whereas Cy7.5 conjugates show very low water solubility and as a result a very low yield. Also, the AF750 conjugates show strongest fluorescence efficacy. Therefore, the AF750 conjugates were further investigated for their in vitro and in vivo characteristics. 
Table 4.1 Characteristics of the synthesized antagonist NIRF-linker-Sta-BBN

\begin{tabular}{|c|c|c|c|c|c|}
\hline Compounds & $\begin{array}{c}\text { Solubility } \\
\text { in } \mathrm{H} 20\end{array}$ & Yield & $\begin{array}{c}\text { Retention } \\
\text { time }\end{array}$ & $\begin{array}{c}\text { HPLC } \\
\text { purification }\end{array}$ & $\begin{array}{c}\text { Measured } \\
\text { MW } \\
\text { (theoretical } \\
\text { MW) }\end{array}$ \\
\hline AF750-G-Pip-Sta-BBN & Good & High & $12.3 \mathrm{~min}$ & Easy separation & $\begin{array}{l}2176.9 \\
(2177)\end{array}$ \\
\hline AF750-GSG-Sta-BBN & Good & High & $13.1 \mathrm{~min}$ & Easy separation & $\begin{array}{l}2180.8 \\
(2181)\end{array}$ \\
\hline AF750-6Ahx-Sta-BBN & Good & High & $14.6 \mathrm{~min}$ & Easy separation & $\begin{array}{l}2092.4 \\
(2093)\end{array}$ \\
\hline DL800(PEG)-G-Pip-Sta-BBN & Good & Average & $17.2 \mathrm{~min}$ & $\begin{array}{c}\text { Hard } \\
\text { separation }\end{array}$ & $\begin{array}{l}2836.08 \\
(2836.1)\end{array}$ \\
\hline DL800(PEG)-GSG-Sta-BBN & Good & Average & $18.1 \mathrm{~min}$ & $\begin{array}{c}\text { Hard } \\
\text { separation }\end{array}$ & $\begin{array}{l}2839.2 \\
(2840)\end{array}$ \\
\hline DL800-6Ahx-Sta-BBN & Good & Average & $>25 \min$ & $\begin{array}{c}\text { Hard } \\
\text { separation }\end{array}$ & $\begin{array}{c}2117.0 \\
(2117.0)\end{array}$ \\
\hline Cy7.5-G-Pip-Sta-BBN & Bad & Low & -- & Easy separation & 1941.1 \\
\hline Cy7.5-GSG-Sta-BBN & Bad & Low & -- & Easy separation & $\begin{array}{c}1945.4 \\
(1945.0)\end{array}$ \\
\hline Cy7.5-6Ahx-Sta-BBN & Bad & Low & -- & Easy separation & $(1857.0)$ \\
\hline
\end{tabular}

Figure 5.2 shows the molecular structures of three AF750-linker-Sta-BBN antagonist analogs. The compounds have slightly different retention time on HPLC (Figure 5.3). AF750-G-Pip-Sta-BBN has the shortest retention time, $12.3 \mathrm{~min}$, implying the highest hydrophilicity. The AF750-6Ahx-Sta-BBN has the longest retention time, 14.6min, implying the highest hydrophobicity, and the AF750-GSG-Sta-BBN has a retention time of $13.1 \mathrm{~min}$. The molecular weight (MW) is 2176.9 for AF750-G-pip-StaBBN, 2180.8 for AF750-GSG-Sta-BBN, and 2092.4 for AF750-6Ahx-Sta-BBN determined by mass spectroscopy, in agreement with the theoretical estimated MW (Figure 5.4). Figure 5.5 shows the absorption spectrum and fluorescence spectrum of AF750-G-Pip- 
Sta-BBN, with the absorption maximum at $751.4 \mathrm{~nm}$ and the emission peak at $774.5 \mathrm{~nm}$.

The result is in good agreement with the fluorescence profile for AF750 (excitation peak at $749 \mathrm{~nm}$ and emission peak at $775 \mathrm{~nm})$, confirming that the conjugation of the BBN antagonist to AF750 did not alter its fluorescence property.

(A) AF750-G-Pip-Sta-BBN

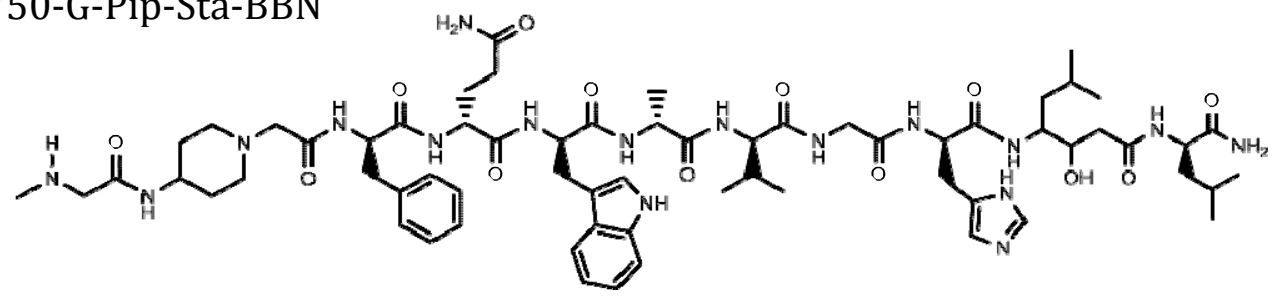

(B) AF750-GSG-Sta-BBN<smiles>CNCC(=O)N[C@@H](CO)C(=O)NCC(=O)N[C@@H](Cc1ccccc1)C(=O)N[C@@H](CCC(N)=O)C(=O)N[C@@H](Cc1c[nH]c2ccccc12)C(=O)N[C@@H](C)C(=O)N[C@H](C(=O)NCC(=O)N[C@@H](Cc1cnc[nH]1)C(=O)N[C@@H](CC(C)C)C(O)CC(=O)NCC(=O)NC)C(C)C</smiles>

(C) AF750-6Ahx-Sta-BBN

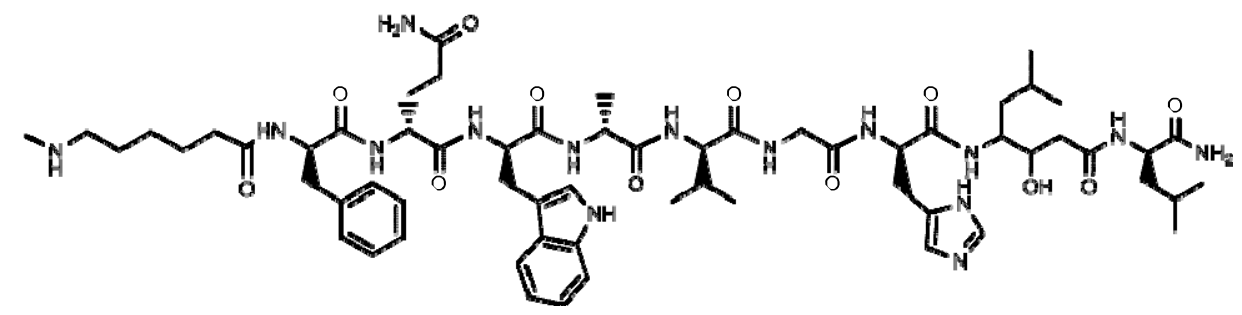

Figure 5.2 Molecular structures of AF750-linker-Sta-BBN analogs. (A) AF750-G-Pip-StaBBN, (B) AF750-GSG-Sta-BBN, and (C) AF750-6Ahx-Sta-BBN 


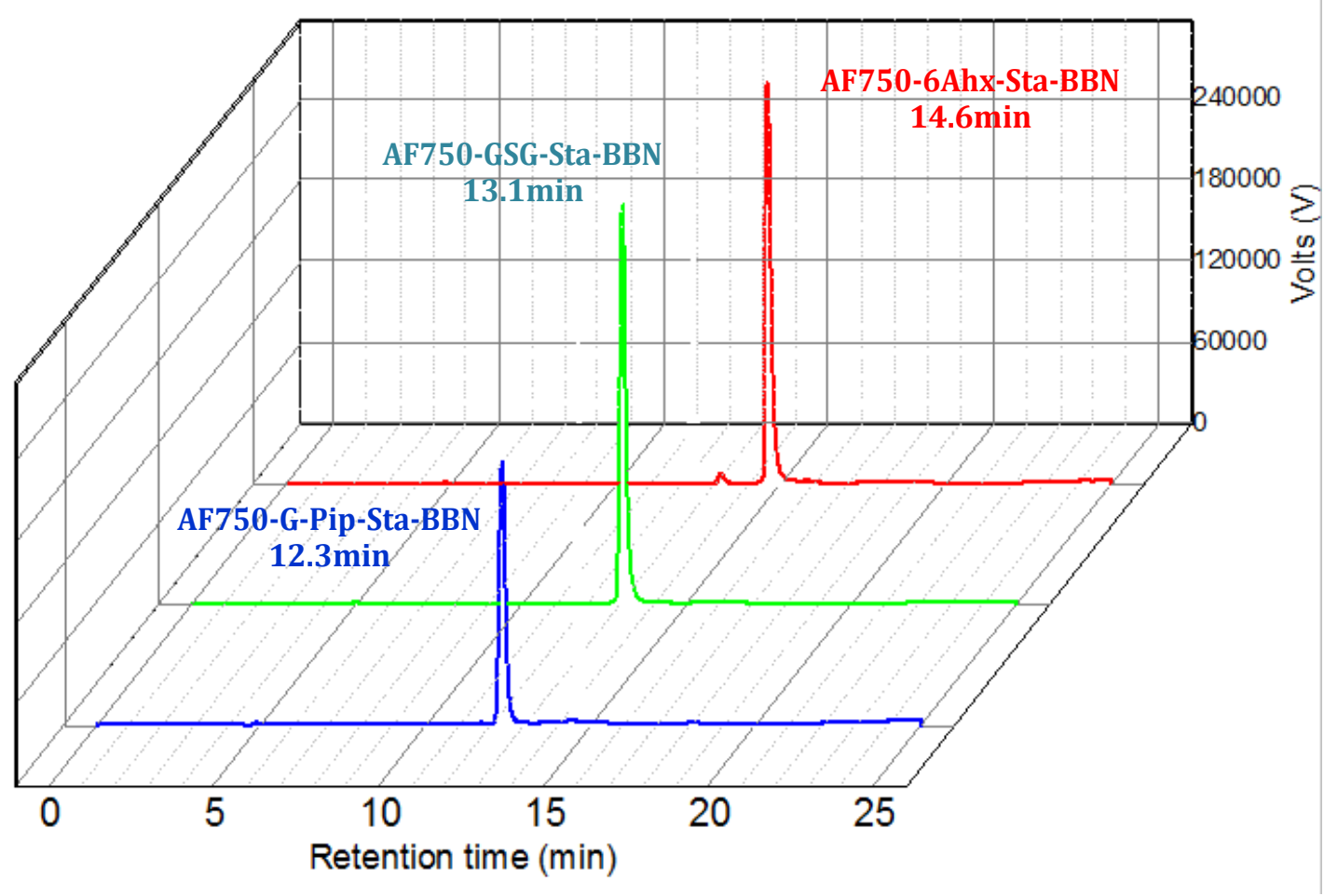

Figure 5.3 HPLC profiles of the purified AF750 labeled Sta-BBN antagonist analogs. HPLC gradient: $20 \%-40 \%$ B buffer in $15 \mathrm{~min}$, flow rate $1 \mathrm{ml} / \mathrm{min}, \mathrm{A}$ buffer: 0.1 TFA in H2O, B buffer: 0.1 TFA in acetonitrile (ACN). 
AF750-G-Pip-Sta-BBN (MW: 2176.9)

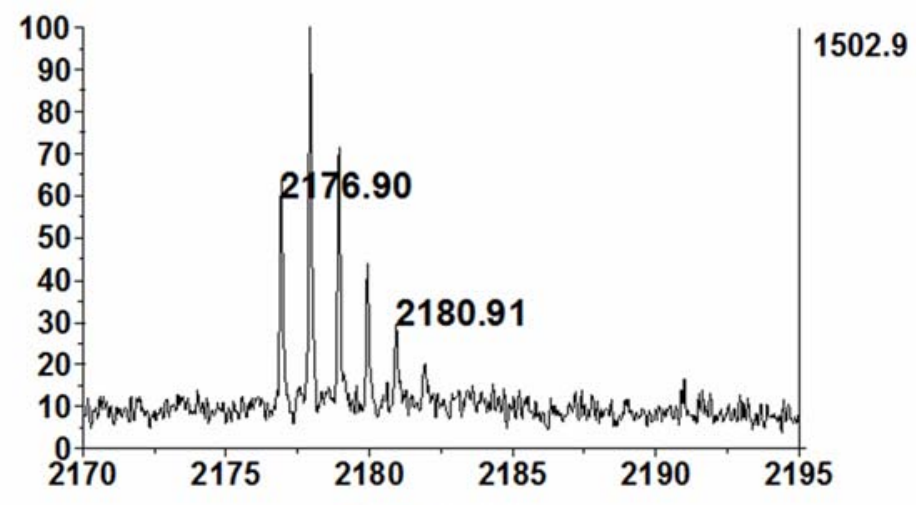

AF750-GSG-Sta-BBN (MW: 2180.8)

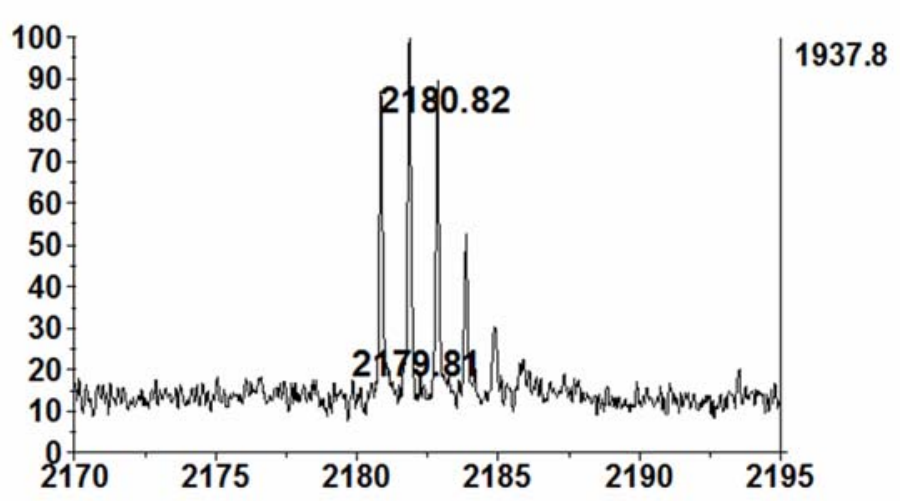

AF750-6Ahx-Sta-BBN (MW:

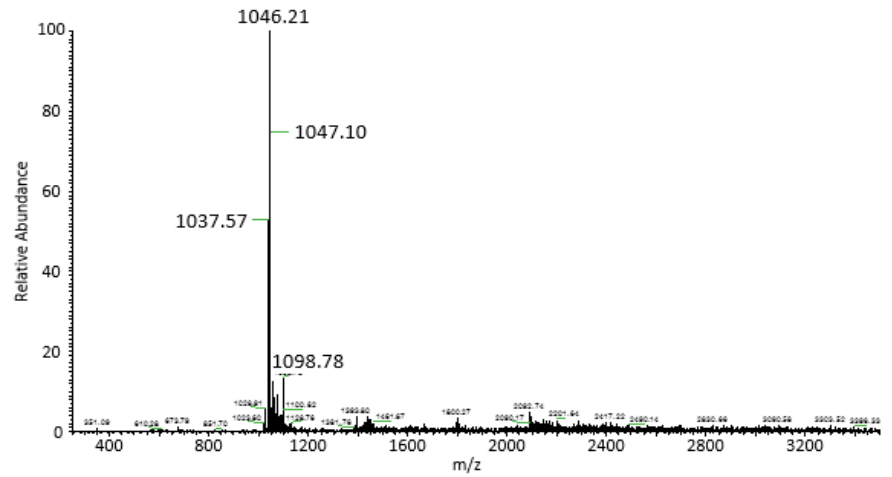

Figure 5.4 Mass spectra of AF750-G-pip-Sta-BBN (theoretical MW: 2177), AF750-GSGSta-BBN (theoretical MW: 2181) and AF750-6Ahx-Sta-BBN (theoretical MW: 2093) 


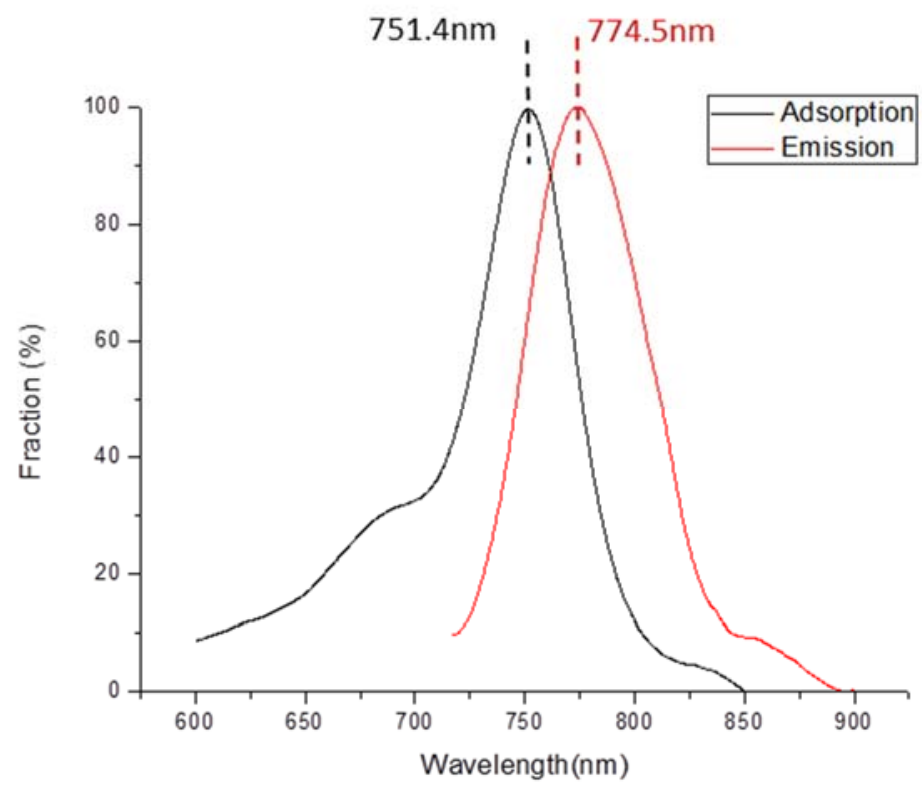

Figure 5.5 Fluorescence spectrum of AF750-G-Pip-Sta-BBN

\section{In vitro Study}

Cellular uptake, blocking and internalization assay. The binding specificities and antagonism were tested for the three compounds, AF750-G-Pip-Sta-BBN, AF750-GSGSta-BBN, and AF750-6Ahx-Sta-BBN, in PC-3 cells. Figure 5.6 shows the NIRF microscopic images. All three compounds displayed a high uptake and a strong fluorescence intensity in the PC-3 cells (left column in Figure 5.6), whereas in the blocking study, there was little fluorescence signal in the PC-3 cells (middle column in Figure 5.6), indicating a high binding selectivity of the compounds to the GRP receptors overexpressed on PC-3 cells. In the internalization study, the cell surface bound ligands were washed off using acidic buffers; only weak fluorescence signals could be found 
around the cells (right column in Figure 5.6), indicating the compounds were not internalized into the PC-3 cells, which is an important character of antagonistic properties for BBN antagonist. Also, the cell's morphology looks normal after treatment with these BBN analogs, implying a low level of toxicity.

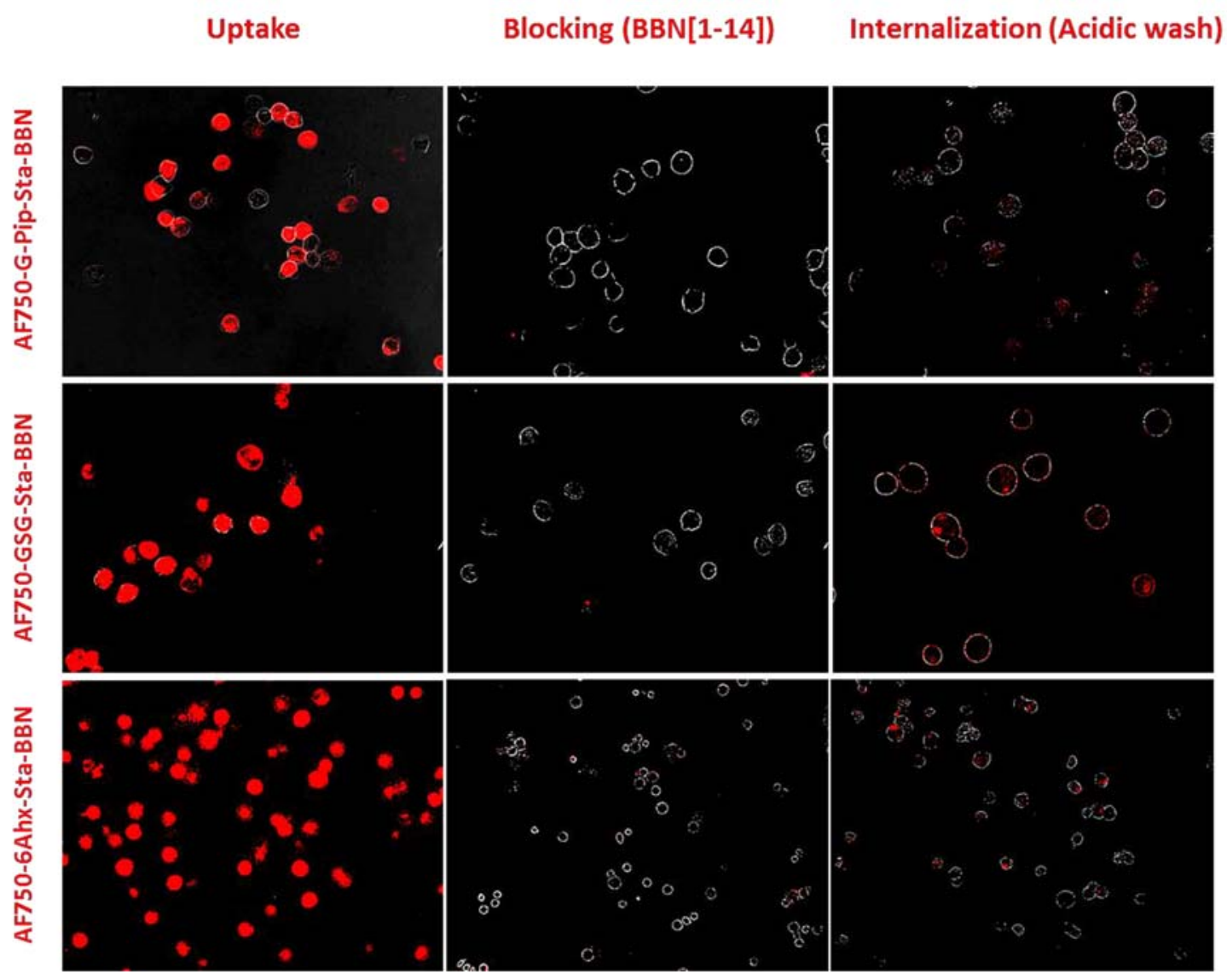

Figure 5.6 In vitro NIRF microscopic images overlaid on the bright-field images for PC-3 cells treated with AF750-G-Pip-Sta-BBN (top row), AF750-GSG-Sta-BBN (middle row), and AF750-6Ahx-Sta-BBN (bottom row) in the uptake study (left), the blocking study (middle) and the internalization study (right). The red represents AF750 fluorescence signals.

Calcium mobilization study. Calcium mobilization study was performed to examine further the antagonism of the AF750 labeled bombesin antagonist analogs. As 
Figure 5.7 shows, BBN[1-14], a bombesin agonist, was able to induce a strong intracellular calcium mobilization, even when the incubation quantity of BBN[1-14] was as low as $10^{-9} \mathrm{M}$ for $40,000 \mathrm{PC}-3$ cells. On the other hand, all three AF750 labeled BBN antagonist analogs induced little or none calcium response in the PC-3 cells, even when the quantity to treat these cells was more than $10^{-5} \mathrm{M}$, validating the antagonistic properties of the compounds.

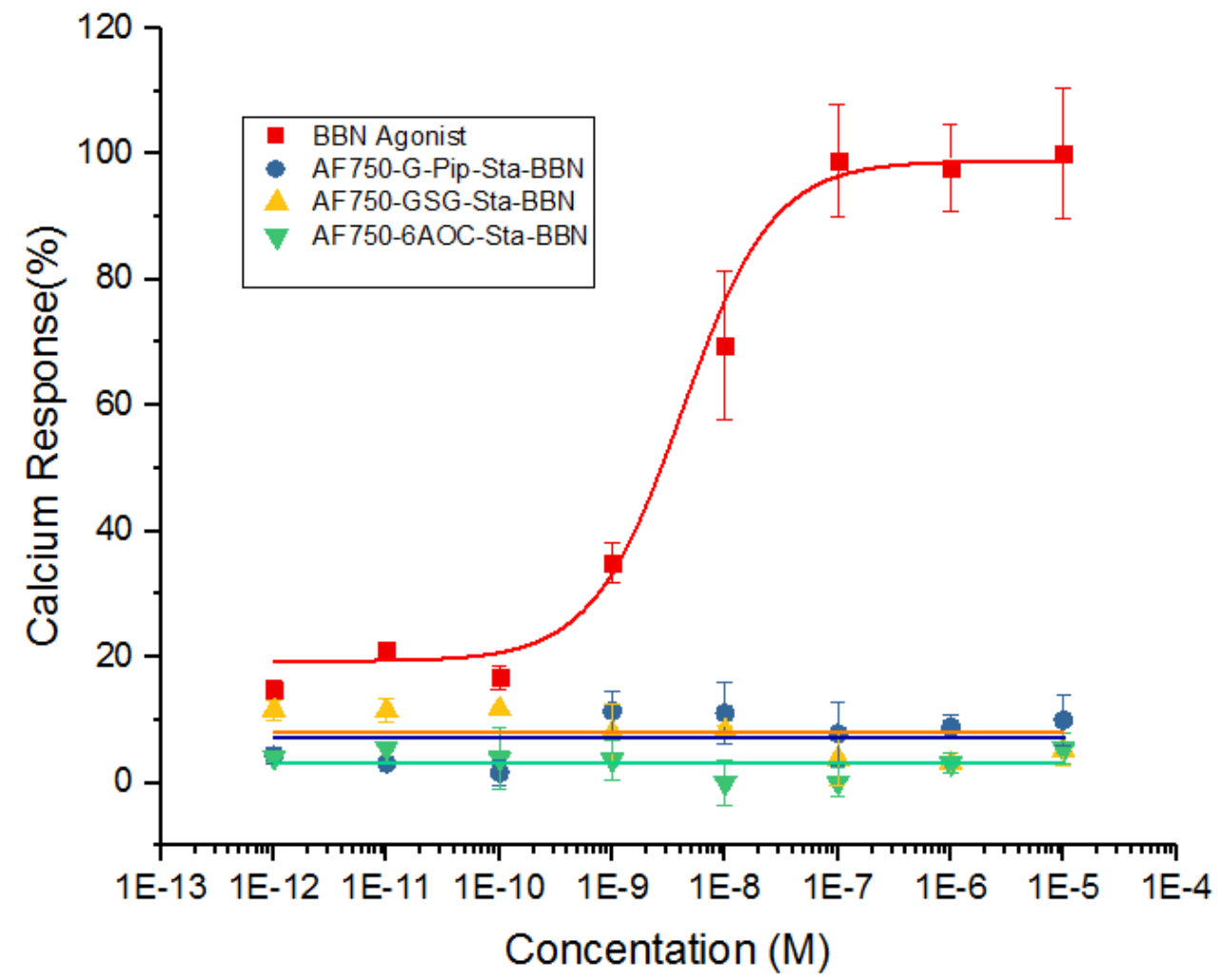

Figure 5.7 The fitting curves for calcium mobilization study for BBN[1-14] (BBN agonist) and AF750 labeled BBN antagonist

Binding affinity determination. The IC50 values of AF750-G-Pip-Sta-BBN and AF750-GSG-Sta-BBN against ${ }^{125}$ I-Tyr ${ }^{4}$-BBN are $9.8 \pm 2.9 \mathrm{nM}$ and $16.5 \pm 4.6 \mathrm{nM}$, 
respectively, determined in PC-3 cells (Figure 5.8), indicating a very high binding affinity to the GRP receptors overexpressed by PC-3 cells.

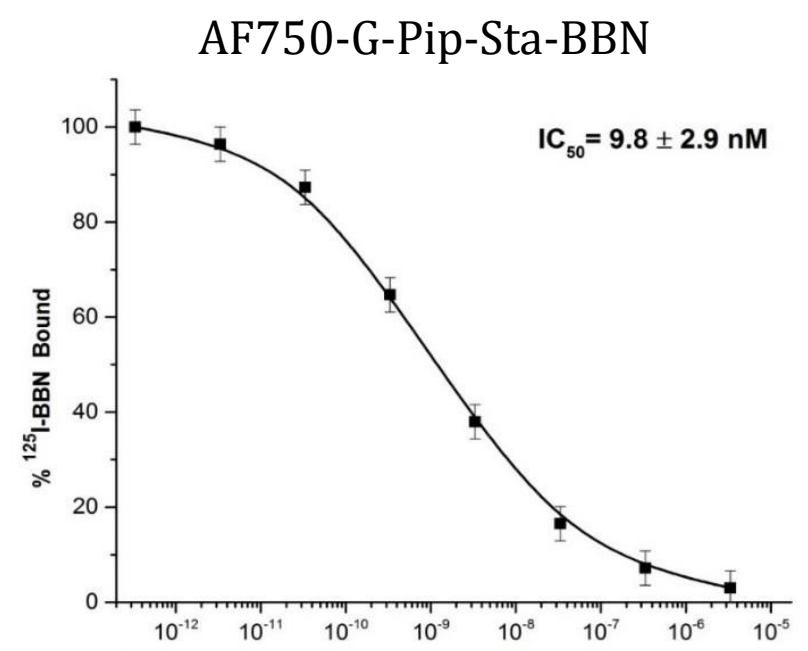

Molar Concentration of AF750-Gly-4Amino Carboxymethyl-Piperidine-RM2

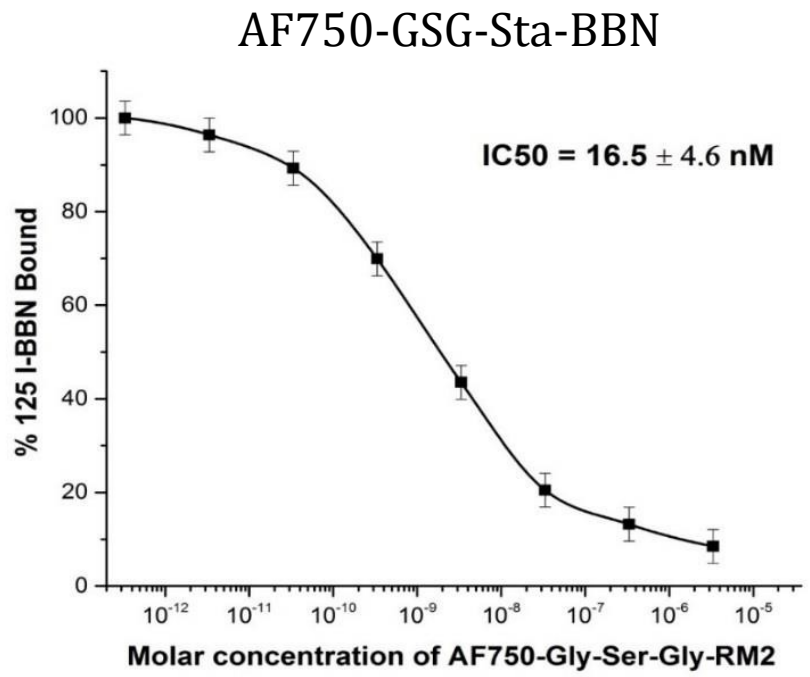

Figure 5.8 IC50 value of AF750-G-Pip-Sta-BBN/ and AF750-GSG-Sta-BBN against 125ITyr4-BBN in PC-3 cells is $9.8 \pm 2.9 \mathrm{nM}$ and $16.5 \pm 4.6 \mathrm{nM}$, respectively. 


\section{In vivo and ex Vivo NIRF Imaging Study}

Selection of optimal compounds for in vivo studies. In a preliminary in vivo test with Cy7.5-6Ahx-Sta-BBN, the compound did not go through the tail into the main body, probably due to the bad water solubility. In the in vivo preliminary test with DL800$6 \mathrm{Ahx}-\mathrm{Sta}-\mathrm{BBN}$, the mouse in the blocking group died at $1 \mathrm{hr} 40 \mathrm{~min}$ post injection (p.i.) possibly due to the adverse effect of the BBN[1-14], and compounds were found to be stuck to every organ by the ex vivo imaging. The other mice in the uptake group stayed alive during the NIRF imaging study. The bio-distribution result at $27 \mathrm{hr}$ p.i. shows that most of the compounds accumulated in the liver, probably due to the relatively high hydrophobicity, but all the other organs also have detectable bio-distribution of the compound. Due to the low reaction yield, unfavourable water solubility and unfavorable bio-distribution data in the preliminary in vivo study, further studies with Cy7.5 and DL800(-PEG) labeled bombesin antagonist analogs were laid aside, and the rest of this work will be focused on exploring the performance of AF750 labeled bombesin antagonist analogs in the prostate tumor xenograft bearing mouse model.

Dosage and time course determination No adverse effects and toxicity were observed for mice at all doses (0.44 - $39.1 \mathrm{nmol} /$ per mouse) tested for up to 25 -hour post tail vein injections with any of the three AF750 labeled Sta-BBN antagonist analogs in SCID and CF1 mice. At dosages higher than $10 \mathrm{nmol}$, mice showed an elevated tumor to muscle ratio in the in vivo IVIS imaging study. However, tumors in the uptake group did not exhibit significantly higher signal intensities than that in the blocking group in the ex vivo imaging study at 20 hours or at 1-hour p.i. in the study with AF750-GSG-Sta- 
BBN. In this case, the dosage of the compounds was thought to be too high that they could even kick out the BBN[1-14] that occupied the GRP receptors, and therefore, the contrast of tumor signals between the uptake groups and the blocking groups attenuated. The dosage of the agents was then cut down to be lower than $2.2 \mathrm{nmol}$, or even lower than $0.5 \mathrm{nmol}$ for the in vivo NIRF imaging analyses.

In vivo NIRF imaging analysis. The in vivo NIFR imaging evaluation was conducted at $15 \mathrm{~min}, 30 \mathrm{~min}$, one $\mathrm{hr}$, two $\mathrm{hr}$ and/or $3 \mathrm{hr}$ post injection at a very low dose $(0.44$ $\mathrm{nmol}$ or $2.19 \mathrm{nmol}$ per mouse) for each of three AF750 labeled antagonists in mice grouped in the uptake and blocking groups. Figure 5.9 shows the in vivo NIRF images at 1-hour post tail vein injection of the compounds. The tumor NIRF signal intensity was significantly higher in the uptake group (Figure 5.9A,C,E) than that in the blocking group (Figure 5.9B,D,F) for each of the three compounds, respectively. The tumor size and anatomical structure were assessed by MRI and shown on a cross-section T2-weighted MRI image for each of the mouse (Figure 5.9G - L). 


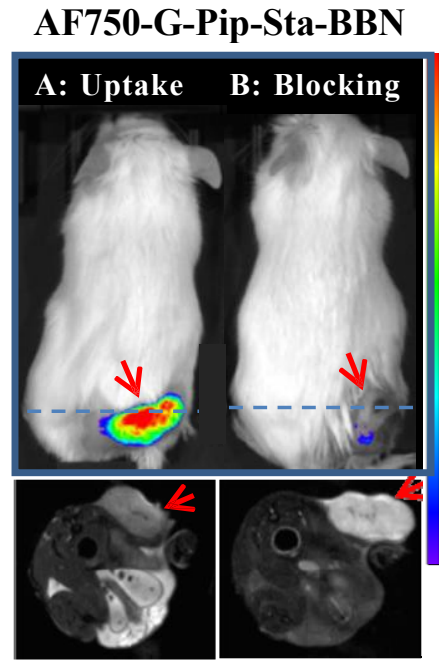

T2W-MRI

Dose: $0.44 \mathrm{nmol}$

Blocker: $50 \mu \mathrm{g}$ BBN[1-14]
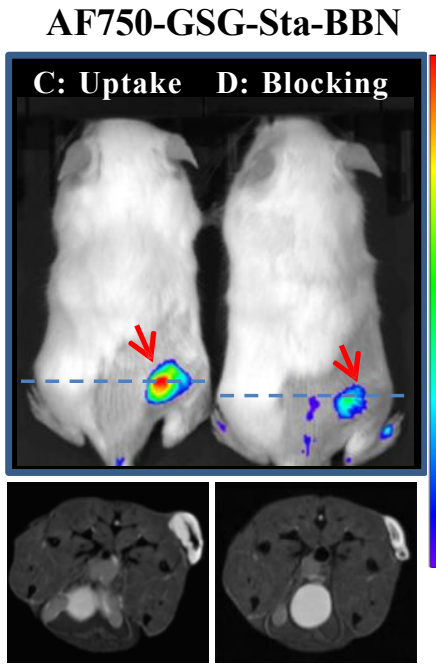

T2W-MRI

Dose: $2.19 \mathrm{nmol}$

Blocker: $75 \mu \mathrm{g}$ BBN[1-14]

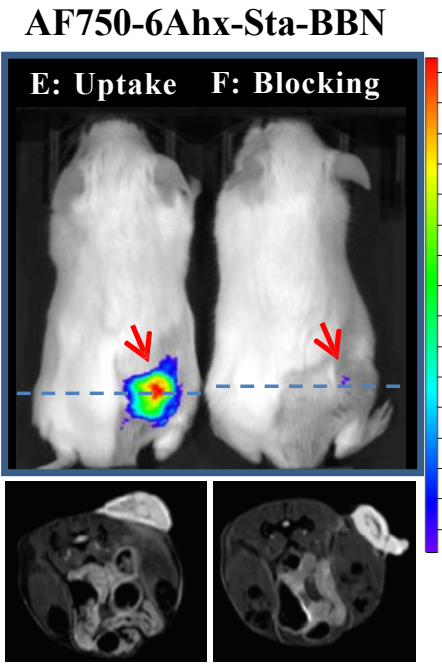

T2W-MRI

Dose: $0.44 \mathrm{nmol}$

Blocker: $50 \mu \mathrm{g}$ BBN[1-14]

Figure 5.9 In vivo NIRF imaging (Ex: $745 \mathrm{~nm} / \mathrm{Em}: 800 \mathrm{~nm}$ ) of SCID mice bearing PC-3 tumor xenograft on the right flank at 1 hour post tail vein injection of AF750-G-Pip-Sta-BBN (left), AF750-GSG-Sta-BBN (middle) and AF750-6Ahx-Sta-BBN (right), respectively, for the uptake group $(A, C, E)$ as compared to the blocking group $(B, D, F)$. Corresponding T2-weighted MRI of the tumor cross section is shown below each NIRF image (G-L). Dotted line indicates the MRI cross section location.

The tumor NIRF intensity was measured at each time point and shown in Figure

5.10. The blocking study with a pre-injection of unlabeled full-length BBN[1-14] showed a fairly effective blocking of the GRP receptor binding as indicated by the significantly decreased tumor signals at 15 min up to $2 \mathrm{~h}$ time points for AF750-G-pip-Sta-BBN, and at 30 min up to 2 or $3 \mathrm{~h}$ for AF750-GSG-Sta-BBN and AF750-6Ahx-Sta-BBN, as compared to the uptake group. 

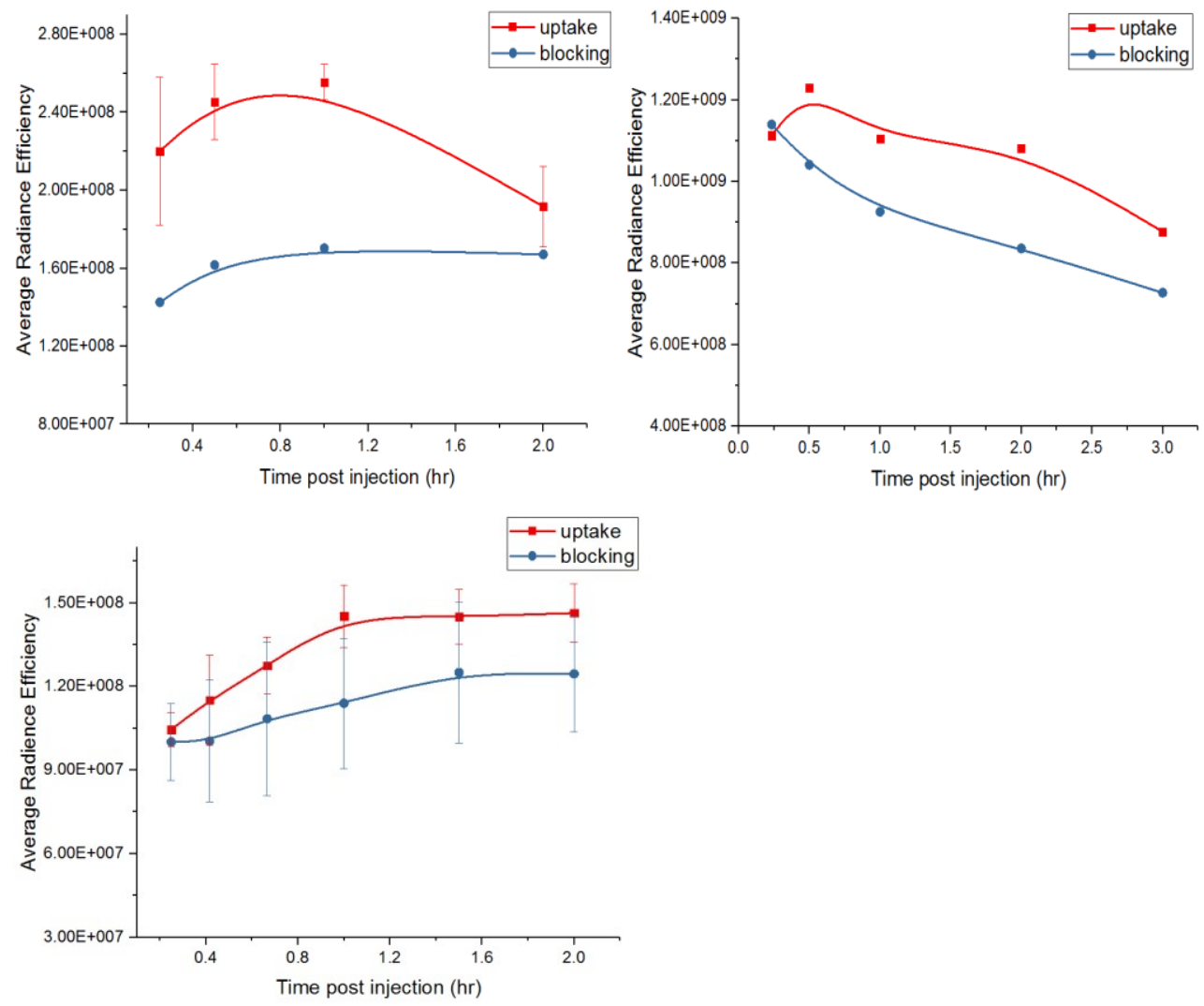

Figure 5.10 In vivo kinetic tumor NIRF signal strength(Ex: $745 \mathrm{~nm} / \mathrm{Em}: 800 \mathrm{~nm})$ of SCID mice bearing PC-3 tumor xenograft on the right flank for AF750-G-Pip-Sta-BBN (A)(N=2 for the uptake and $\mathrm{N}=1$ for the blocking), AF750-GSG-Sta-BBN (B)(N=1 for the uptake and $\mathrm{N}=1$ for the blocking) and AF750-6Ahx-Sta-BBN (C) ( $\mathrm{N}=3$ for the uptake and $\mathrm{N}=3$ for the blocking), respectively.

Ex vivo NIRF imaging and biodistribution analysis. In the ex vivo analysis, all mice were sacrificed at $2.5 \mathrm{hr}$ post injection, except the mice in the AF750-GSG-Sta-BBN study that were sacrificed at $3.5 \mathrm{hr}$ post injection. The NIRF imaging was performed immediately after the tissue collection. As Figure 5.11 shows, tumor and pancreas in the uptake group displayed significantly higher signals in the uptake group than the counterparts in the blocking group for all three compounds, while muscle and normal 
prostate tissue showed little or no fluorescence signals. It is noted that pancreases is one of few normal organs with GRP receptors overexpression. ${ }^{157}$ These ex vivo results validated the in vivo NIRF imaging analysis and indicate a high in vivo binding specificity of the AF750 labeled bombesin antagonist analogs to GRP receptors that overexpressed in PC-3 tumors and pancreas.

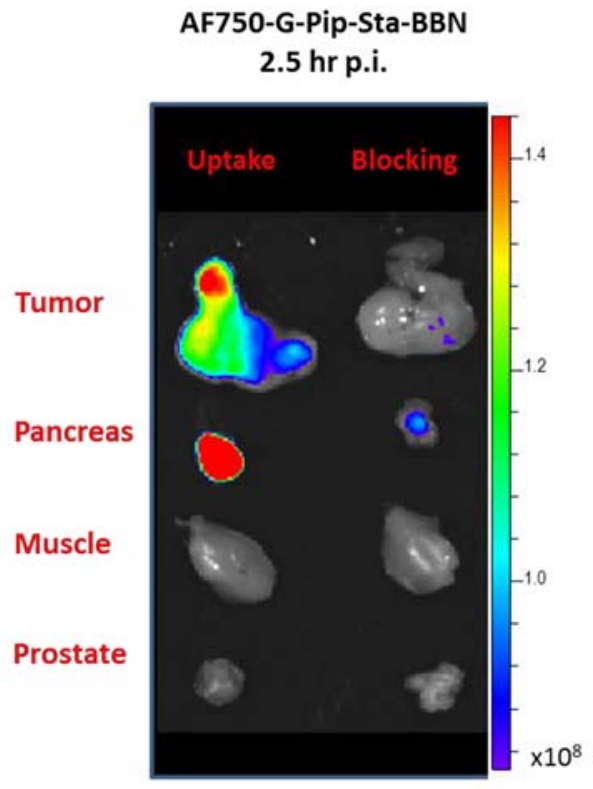

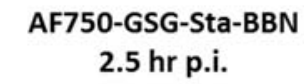

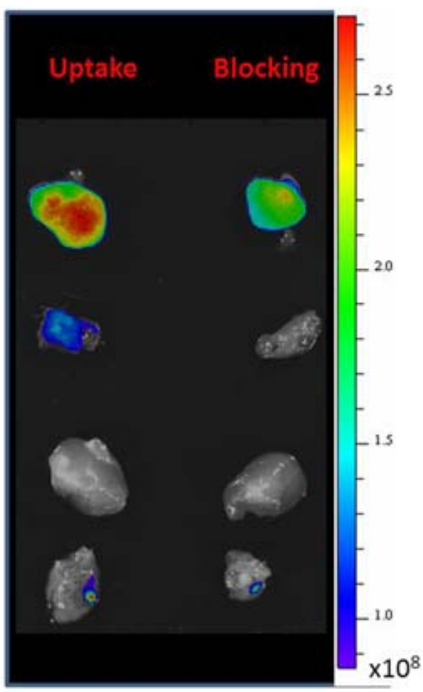

AF750-6Ahx-Sta-BBN

2.5 hr p.i.

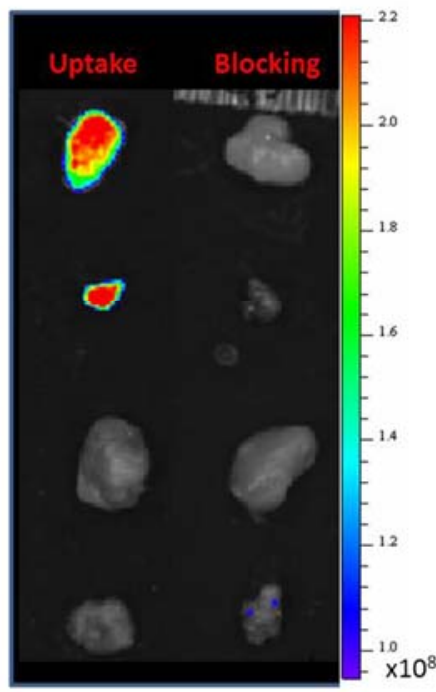

Figure 5.11 Ex vivo NIRF imaging (Ex: $745 \mathrm{~nm} / \mathrm{Em}: 800 \mathrm{~nm}$ ) of tumor, pancreas, muscle, and prostate in the uptake group as compared to the blocking group for AF750-G-PipSta-BBN (left), AF750-GSG-Sta-BBN (middle), and AF750-6Ahx-Sta-BBN (right). The color bar represents the average radiance efficiency $\left(\frac{\mathrm{p} / \mathrm{sec} / \square \mathrm{m}^{2} / \mathrm{sr}}{}\right.$ $\mu W /$ 回 $m^{2} \quad$.

The NIRF bio-distribution studies are shown in Figure 5.12, Figure 5.13, and Figure 5.14 for each of the three compounds, respectively. The fluorescence measurements of multiple organs revealed that urines displayed much higher signal intensities than all of other tissues, and at the higher dose $(2.19 \mathrm{nmol})$ the kidney also showed a much higher signal than other organs, indicating the renal-urine excretion 
route of the compounds. Among all of the tissues, tumor and pancreas are the only two tissues showed a significantly higher signal intensity in the uptake group than that in the blocking group ( $p<0.5)$ (Figure 5.12 - Figure 5.14), quantitatively confirming the high in vivo binding affinity and specificity of each compound to the GRP receptors. On the other hand, normal prostate tissue, brain, heart, spleen, muscle, small and large intestines showed very low fluorescence signals, indicating there is no non-specific binding of the compounds in these organs hence minimizing potential toxicities. Liver, lungs, bladder, kidneys, and urine showed slightly higher signals in the blocking group than that in the uptake group, due to the blocked GRPr binding sites by BBN[1-14] in the blocking group. The stomach also showed an elevated fluorescence signal in both groups, likely due to some degree a GRPr expression in the gastric fluids. ${ }^{158}$ There was little detectable blood fluorescence at 2.5 hours for the $0.44 \mathrm{nmol}$ dose or 3.5 hours for the $2.19 \mathrm{nmol}$ dose, indicating the clearance of the compounds in the blood circulation. 


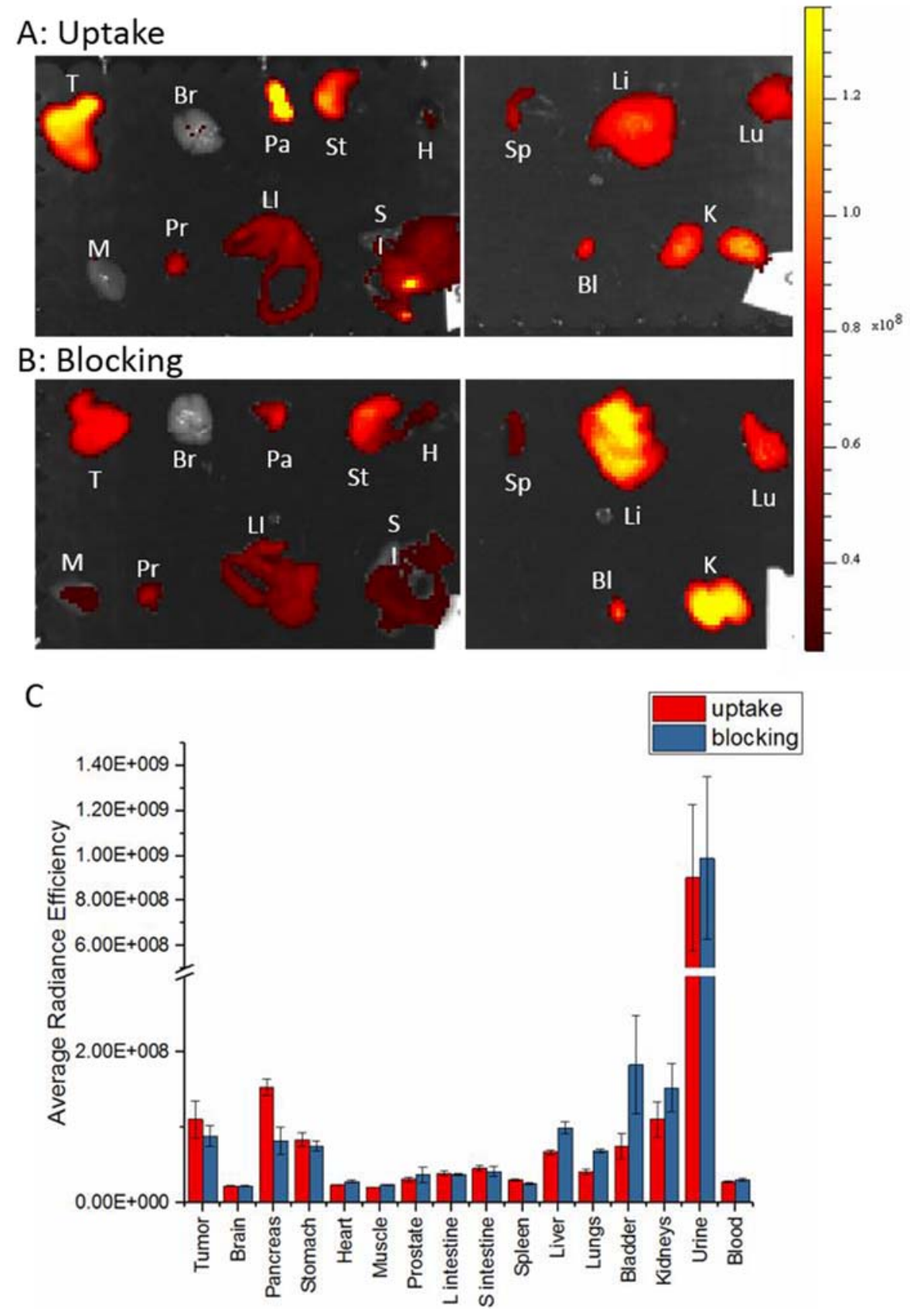

Figure 5.12 Ex vivo NIRF image of (A) uptake and (B) blocking representative animals, and (C) bio-distribution data for AF750-G-Pip-Sta-BBN at 2.5 hour p.i. * indicates a significant difference by the student t-test, $\mathrm{p}<0.5$. Letters denote: $\mathrm{T}$ : Tumor, Br: Brain, Pa: Pancreas, St: Stomach, H: Heart, M: Muscle, Pr: Prostate, LI: Large intestine, SI: Small intestine, Sp: Spleen, Li: Liver, Lu: Lungs, BI: Bladder, K: Kidneys 

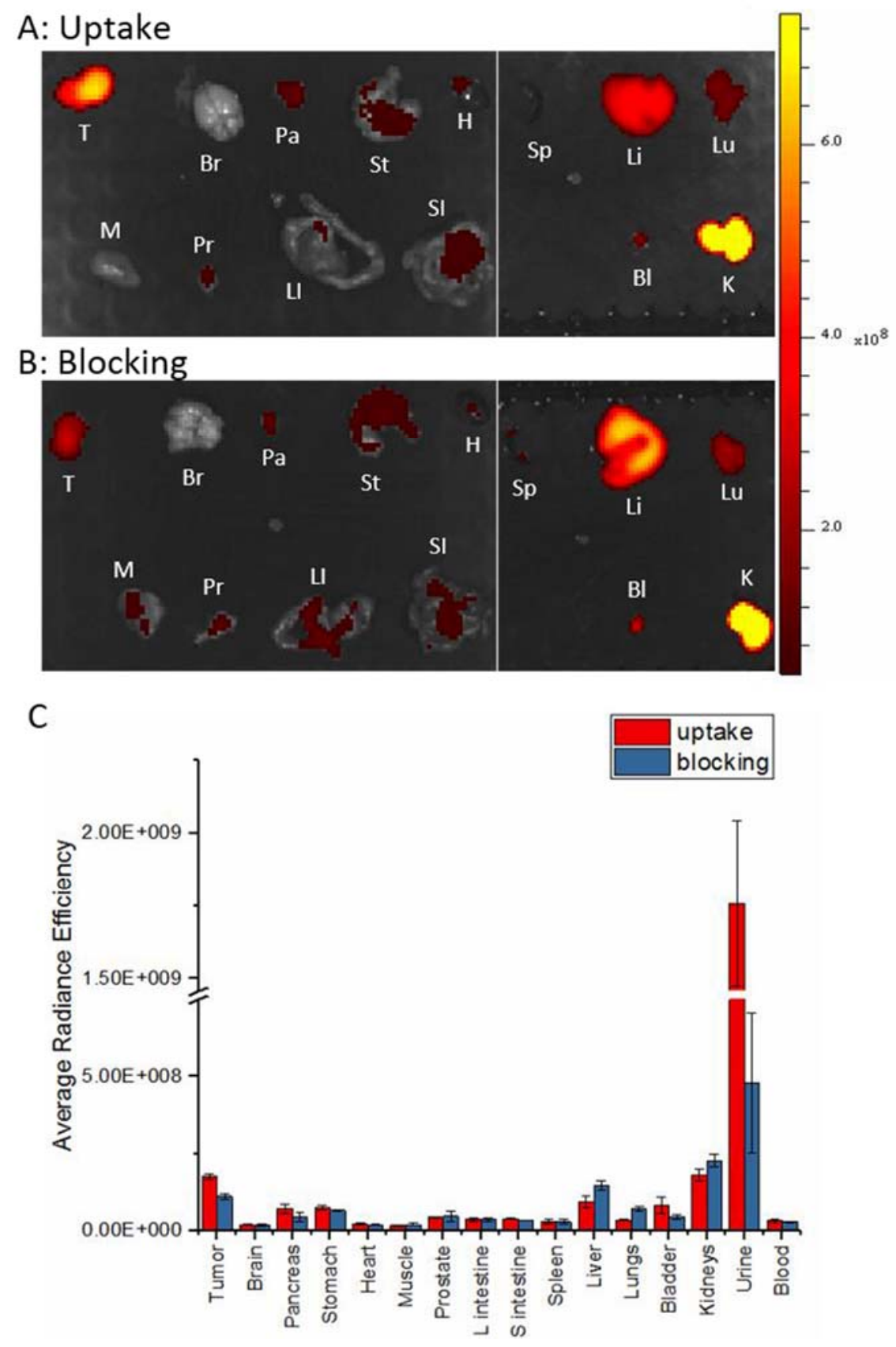

Figure 5.13 Ex vivo NIRF image of (A) uptake and (B) blocking representative animals, and (C) bio-distribution data for AF750-GSG-Sta-BBN at 3.5 hours p.i. * indicates a significant difference by the student t-test, $p<0.5$. Letters denote: T: Tumor, Br: Brain, Pa: Pancreas, St: Stomach, H: Heart, M: Muscle, Pr: Prostate, LI: Large intestine, SI: Small intestine, Sp: Spleen, Li: Liver, Lu: Lungs, BI: Bladder, K: Kidneys 


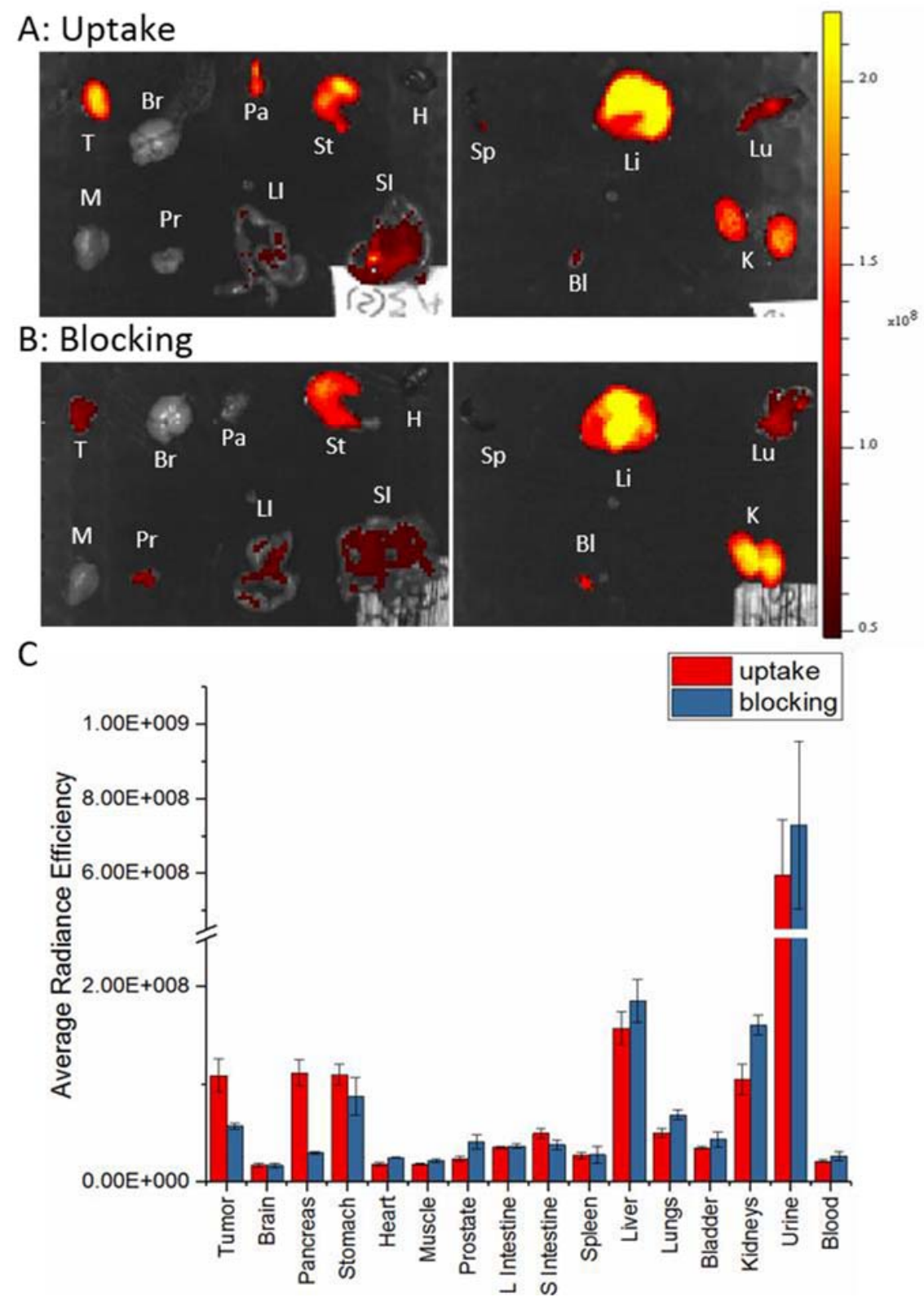

Figure 5.14 Ex vivo NIRF image of (A) uptake and (B) blocking representative animals, and (C) bio-distribution data for AF750-6Ahx-Sta-BBN at 2.5-hour p.i. * indicates a significant difference by the student t-test, $\mathrm{p}<0.5$. Letters denote: $\mathrm{T}$ : Tumor, Br: Brain, Pa: Pancreas, St: Stomach, H: Heart, M: Muscle, Pr: Prostate, LI: Large intestine, SI: Small intestine, Sp: Spleen, Li: Liver, Lu: Lungs, BI: Bladder, K: Kidneys 


\section{Histological study}

The in vivo binding specificity of the three AF750 labeled antagonist analogs to the tumor cells was further confirmed by histological studies. As shown in Figure 5.15, the fluorescence dye was only detected in the tumor tissue slices in the uptake groups, but not in the blocking groups, confirming the specific binding of the AF750 labeled bombesin antagonist analogs to the PC-3 tumor on a microscopic level. Furthermore, normal prostate tissue and muscle tissue slices had no detectable fluorescence dyes, indicating no non-specific bindings of the compounds in these organs. 


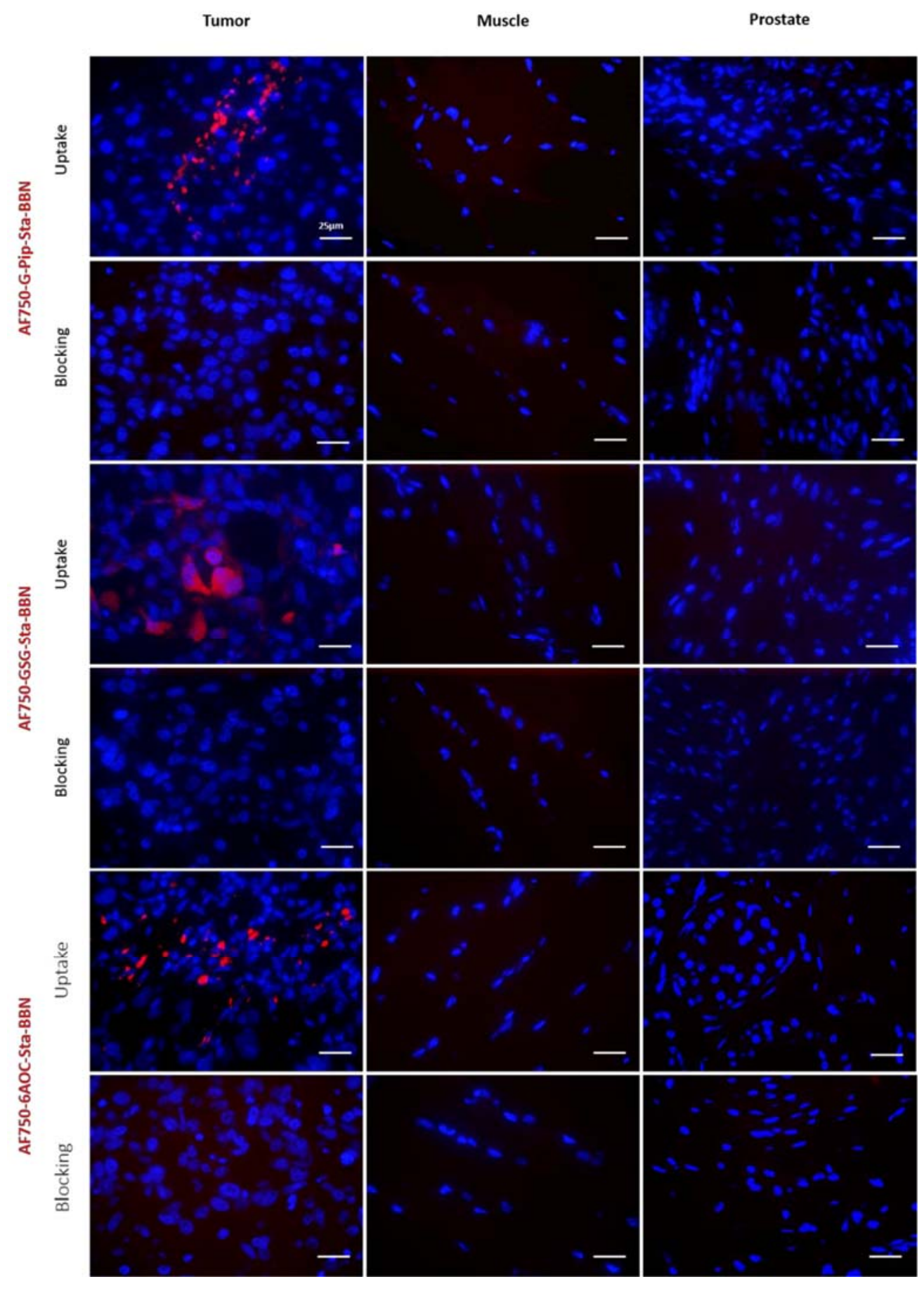

Figure 5.15 Histological studies for the tumor, muscle, and prostate tissue slices in the uptake and blocking group for AF750-G-pip-Sta-BBN (first two rows), AF750-GSG-StaBBN (middle two rows) and AF750-6Ahx-Sta-BBN (last two rows). Red: AF750 dye; Blue: DAPI staining. 


\section{Discussion}

GRP receptor antagonists were reported to have high binding affinities with desirable pharmacokinetics and biodistribution as compared to the bombesin agonists to GRPr overexpressed by prostate cancer cells. ${ }^{7}$ In this work, BBN antagonists labeled with NIR fluorescent dyes, AF750, DL800(-PEG), Cy7.5, were synthesized and explored as potential novel NIRF molecular imaging probes for specific prostate cancer detection and targeted therapy. Their specificities and binding affinities to GRP receptors overexpressed on prostate cancer were evaluated in the cellular experiment and the mouse model of human prostate cancer. Since properties, such as hydrophilicity, fluorescent efficiency, and signal penetration depth of the compound strongly rely on the fluorescence dye, three different dyes, AF750, DL800/DL800-PEG, and Cy7.5, were initially chosen for conjugation with the bombesin antagonists for a preliminary screening of an optimal dye. Furthermore, the dye moiety that directly links to the Nterminus of peptide would interfere with the binding efficacy, so a pharmacokinetic modifier or a linker is needed between them to enable the preservation of biological integrity and receptor specificity of the bombesin antagonists. ${ }^{127}$ In this study, Gly-SerGly (GSG) was selected as a long flexible linker, ${ }^{128}$ while Gly4Amino_Carboxymethyl_Piperidine (G-pip) was selected as a positively charged linker that may be beneficial to its binding to the negatively charged cellular membranes, ${ }^{70}$ and 6Ahx (- $\left.\mathrm{NH}\left(\mathrm{CH}_{2}\right)_{5} \mathrm{CO}-\right)$ was selected as a hydrophobic linker that may lead to prolonged blood circulation time, and therefore, enhanced entrapment in tumors. 
A total of nine compounds, with three different dyes, AF750, DL800(-PEG), and Cy7.5, and three different linkers, G-Pip, GSG, and 6Ahx, were synthesized via the EDAC/NHS conjugation chemistry and purified. The three Cy7.5 bombesin antagonist analogs were regarded not qualified for further studies due to their poor water solubility. The DL800/DL800-PEG bombesin antagonist analogs were difficult to be separated and purified from the unconjugated peptides in the HPLC due to the very close retention times of the conjugated and unconjugated peptides. Due to the difficult purification and low yield, we did not pursue further in vivo experiments with the DL800 bombesin antagonist analogs. However, this series of the DL800 BBN antagonist compounds might still be good candidates for site-specific prostate tumor NIRF imaging, since we later obtained a positive result in an in vitro study that proved the specificity of their binding to PC-3 cells. On the other hand, the AF750 bombesin antagonist analogs exhibit the highest fluorescence efficiency, the highest water solubility, the simplest synthesis and purification protocol and the highest reaction yield, and thus, they were studied in the in vitro and in vivo evaluations.

The in vitro study demonstrated a very high binding affinity and specificity of AF750-G-Pip-Sta-BBN, AF750-GSG-Sta-BBN, and AF750-6Ahx-Sta-BBN to the PC-3 prostate cancer cells. They showed the antagonism in which they did not induce any intracellular calcium mobilization as well as were not internalized into the cancer cell. The binding affinity of AF750-G-Pip-Sta-BBN and AF750-GSG-Sta-BBN to GRP receptors, evaluated by the ${ }^{125}$ I-Tyr ${ }^{4}-B B N$ replacement IC50 binding assay, showed similar nM 
values within the uncertainty indicating a very high in vitro binding efficacy of the compounds to the PC-3 cells.

In the in vivo studies, all three compounds showed excellent tumor binding affinity and specificity, and NIRF imaging efficacy in SCID mice bearing PC-3 xenografts (Figure 5.9). I.v. injection of a dose as low as $0.44 \mathrm{nmol}$ per $25 \mathrm{~g}$ mouse or $11 \mathrm{pmol} / \mathrm{kg}$ is sufficient to generate very high in vivo NIRF imaging contrast on the tumor sites at 30 min, one hours and two hours post injection. In the uptake groups, tumor signals appeared the highest at one-hour time point for all three compounds, then decreased rapidly for AF750-G-pip-Sta-BBN and slightly for AF750-GSG-Sta-BBN at two hours time point. The tumor signal remained stable for AF750-6Ahx-Sta-BBN up to two hours time point (Figure 5.10). Furthermore, all three compounds showed rapid urine excretion as indicated by the peaked bladder intensities at $30 \mathrm{~min}$ to $1 \mathrm{~h}$ time points in the uptake groups, and one hour to 1.5 hours in the blocking groups. Among the three compounds, AF750-G-pip-Sta-BBN showed the fastest and AF750-6Ahx-Sta-BBN showed the slowest pharmacokinetics measured by the NIRF signals on the tumor sites, from the background signals due to blood circulation, and on the bladder. This difference can be attributed to the different linker hydrophilicity, with the -G-pip- most hydrophilic and 6Ahx- most hydrophobic.

The ex vivo NIRF imaging at $2.5 \mathrm{~h}$ (Figure 5.11 - Figure 5.14) and the histological studies of the tissue slices (Figure 5.15) confirmed that all three compounds have a very high in vivo binding affinity and specificity to the GRP receptors overexpressed in the 
PC-3 prostate tumors. Comparing the 2.5-hour bio-distribution data of the three compounds, the AF750-6Ahx-Sta-BBN had a relatively higher liver signal than the other two compounds. While AF750-G-pip-Sta-BBN showed lower tumor signal than the pancreas signal, AF750-GSG-Sta-BBN showed a significantly higher tumor signal than the pancreases and other organ signals. These bio-distribution data differences agree with the observed in vivo pharmacokinetics difference among the three compounds and may be explained by the linker properties due to the hydrophilicity and charge groups on the linkers. These findings warrant future in depth investigations.

The results show that AF750 bombesin antagonist analogs with -G-pip-, -GSG-, and -Ahx- linkers are potential candidates for site-specific NIRF molecular imaging of human prostate cancer. Tumor-targeting molecular imaging probes with a high imaging efficacy in the NIRF wavelength are desirable for imaging guided therapy and surgery using the low-cost and high sensitivity NIRF imaging modality. Since some other cancer types, including breast cancer and small cell lung cancer, also overexpress GRP receptors, the applications of these compounds can be extended to these other human cancers. Although no abnormal behavior was observed for up to 27 hours in mice injected with the compounds, more studies need to be conducted to address the short-term and long-term toxicities of these compounds. 


\section{Acknowledgement}

I appreciate Dr. Zongrun Jiang's help in guidance of HPLC operation, and I would

like to thank Dr. Bandari, Rajendra P for his practice in IC50 measurements, Lisa Watkinson and Terry Carmack for SCID mice tumor inoculation and tissue collection, and Li Lee and Dr. Ran Li for the assistance in imaging experiments, and MU Molecular Cytology Core's support in microscope practice. 


\section{Chapter VI. Summary and future work}

Imaging modalities applied for early detections of cancer have approached a choke point due to the inherent limitations in resolutions or sensitivities, and the applications with site selective or biomarker-specific imaging probes are the future trend for improving detection efficacy. Furthermore, the imaging probes assembled with targeting vectors can specifically enhance the contrast of the disease site to the surrounding areas, promising good potentials for early detection of tumors.

To design an imaging probe with targeting capaaacities, the work will include the development of the original materials with excellent imaging potentials, the modification of the original materials to confer good physical, chemical and biological properties, and additions with targeting moieties. Concerning the raw imaging materials, a large number of imaging materials like iron oxide nanoparticles, gold nanoparticles, composite nanoparticles, quantum dots, organic dyes, radioactive chelators are under development in chemistry labs worldwide. To make them compatible for the imaging use in biological environments, as well as versatile for conjugation reactions with other imaging molecules or targeting molecules, materials like polymers, proteins or lipids are frequently used to coat their surfaces. On the other hand, improving drug delivery efficiency with targeted delivery techniques will lead to enhanced dose in the targeted site for a maximum therapeutic or imaging efficacy utilizing cell-specific ligands or 
receptors. In both industrial and academic labs, new targeting molecules are being designed and screened can be peptides, antibodies and small molecules with specific and strong binding to disease sites for cardiovascular diseases, diabetes and especially cancerous tumors without causing severe adverse effects or bringing about strong imaging backgrounds. Due to the large demands about personalized medicine and treatment in the future, companies, especially the leading companies like Pfizer, Roche, Johnson \& Johnson and Bayer are increasingly investing in the drug delivery technology.

My Ph.D. research focuses on developing novel molecular imaging probes for increasingly utilized imaging tools, including MRI, PA tomography, and NIRF imaging, for site-specific and multi-modality medical imaging of prostate tumors and circulating tumors cells. In the first studies, a casein coated iron oxide nanoparticle labeled with BBN-Cy7.5 with the core size of $4.93 \pm 0.42 \mathrm{~nm}$ and the hydrodynamic diameter of 37.3 $\pm 13.8 \mathrm{~nm}$ was developed. The objective was to utilize the nanoparticle based delivery strategy to solve problems regarding enhancing the contrast of the tumor site over normal tissues. The MRI T2 relaxivity of the compound was determined to be $70.2 \mathrm{~s}^{-}$ ${ }^{1} \mathrm{mM}^{-1}$, which was a positive result when core size is only $5 \mathrm{~nm}$. The high binding affinity (IC50 = $2.5 \pm 0.7 \mathrm{nM})$ was confirmed by the in vitro cellular experiment in PC-3 cells. The in vivo study likewise proved a desirable and specific accumulation of the nanoparticles in tumors in a mouse model bearing human PC-3 tumors. In the second studies, an iron oxide nanoparticle with $7.45 \pm 0.88 \mathrm{~nm}$ silica coating loaded with AF750 and agonist BBN was designed and developed for PA imaging of cancerous tumor and circulating tumor cells. Since silica coating is a widely explored method to decorate nanoparticle, 
and the recent report shows that the silica coating can potentially improve the photoacoustic strength of iron oxide nanoparticle, we aimed to adapt this strategy to target prostate tumor for enhanced photoacoustic imaging as well as NIRF imaging. The detection of CTCs has become a recent research focus due to the possibility of predicting or detecting tumors in an early stage. In this case the first phase of the project is successful, and the SIO-AF750-BBN nanoparticle demonstrated a 3-fold increase in the PA contrast enhancement in the tumor tissues in the uptake group as compared to the blocking group, and a 5-fold increase of the PA enhancement as compared to the control group with only the small molecule AF750-G-pip-Sta-BBN injection. The future experiments will include some preliminary experiments with CTCS to explore the possibility of the novel compound to target CTCs and generate distinguishable contrast for CTCS in PA imaging and NIRF imaging. The binding specificity to GRP receptors overexpressed by cancer cells was demonstrated by an in vitro study on PC-3 cells. In the in vivo test with the PC-3 mouse model, results, including the kinetic bio-distribution data in IVIS imaging and PA imaging, the ex vivo outcomes, and the histological outcomes, all demonstrated the specific binding of the compound to GRPr that are overexpressed by prostate tumor and normal pancreas. For the future part of this project, an in vitro study with CTCs in the blood stream will be conducted for investigating the possibility of the compound to target CTCS, although it is not for certain that CTCs also overexpress GRPr. In the third studies, several types of NIRF dyes, including AF750, DL800(-PEG) and Cy7.5, were conjugated with bombesin antagonist (Sta-BBN) via different linkers, Gly-Ser-Gly (GSG), Gly-Pip-Carboxymethyl-Piperidine (G- 
Pip), and $-\mathrm{NH}\left(\mathrm{CH}_{2}\right)_{5} \mathrm{COOH}(6 \mathrm{Ahx})$. The objective was to investigate the outcomes of imaging enhancement of human prostate tumor by a small peptide, especially bombesin antagonist. Bombesin antagonist is a recently discovered peptide sequence that can bind to the surface of the cancer cells with high affinities, and its pharmacokinetics, biodistributions and low toxicity may bring about new scopes of small peptides for molecular imaging applications. The results showed that either cellular experiment or in vivo study proved the specific uptake of the compounds to prostate cancer PC-3 cells or tumor, and IC50 study showed a high binding affinity for both AF750-G-Pip-Sta-BBN (9.8 $\pm 2.9 \mathrm{nM})$ and AF750-GSG-Sta-BBN (16.5 $\pm 4.6 \mathrm{nM})$.

To further explore the possibility of these compounds to be used clinically, these compounds would need to be evaluated in large animal studies and strict toxicity studies. For example, the bombesin antagonist analogs can be studied in details about the possibilities to prevent side effects by bombesin agonist. Since one of the major obstacles of targeted drug delivery techniques to be clinically applied is the toxicity by the new targeting vector, it would be promising for bombesin antagonist to substitute bombesin agonist if it has a better toxicity profile and compatibility to human bodies. The applications of these compounds can be even extended to therapeutic objectives since the modified nanoparticles can offer large loading potentials for therapeutic agents. For example, the casein coated iron oxide nanoparticles can have doxorubicin to be included in the casein matrix by electrical attraction. In the acidic environment surrounding cancer cells, the electrical attraction may be lost and the doxorubicin can be released at a high specificity to cancer cells. Combining therapy with imaging can 
reduce the total dosage of drugs for these two aims, and to offer a unique opportunity to monitor the therapeutic effect to the specific patients. It would be of great importance for progressing personalized imaging and treatment. 


\section{Reference}

(1) Howrey, B. T.; Kuo, Y. F.; Lin, Y. L.; Goodwin, J. S. The journals of gerontology. Series A, Biological sciences and medical sciences 2013, 68, 56.

(2) Hoffman, R. M. New England Journal of Medicine 2011, 365, 2013.

(3) Wei, L.; Li, S.; Yang, J.; Ye, Y.; Zou, J.; Wang, L.; Long, R.; Zurkiya, O.; Zhao, T.; Johnson, J.; Qiao, J.; Zhou, W.; Castiblanco, A.; Maor, N.; Chen, Y.; Mao, H.; Hu, X.; Yang, J. J.; Liu, Z.-R. Mol Imaging Biol 2011, 13, 416.

(4) Wu, X.; Lindner, D.; Yu, G.-P.; Brady-Kalnay, S.; Lu, Z.-R. MR molecular imaging of prostate cancer with a small molecular CLT1 peptide targeted contrast agent. J Vis Exp [Online Early Access]. DOI: 10.3791/50565. Published Online: 2013. (accessed 2013).

(5) Bates, D.; Abraham, S.; Campbell, M.; Zehbe, I.; Curiel, L. PLoS ONE 2014, 9, e97220.

(6) Gibbs, S. L. Quantitative imaging in medicine and surgery 2012, 2, 177.

(7) Cescato, R.; Maina, T.; Nock, B.; Nikolopoulou, A.; Charalambidis, D.; Piccand, V.; Reubi, J. C. Journal of Nuclear Medicine 2008, 49, 318.

(8) Lim, J. C.; Cho, E. H.; Kim, J. J.; Choi, S. M.; Lee, S. y.; Nam, S. S.; Park, U. J.; Park, S. H. Nuclear Medicine and Biology, 42, 131.

(9) Schally, A. V.; Comaru-Schally, A. M.; Nagy, A.; Kovacs, M.; Szepeshazi, K.; Plonowski, A.; Varga, J. L.; Halmos, G. Frontiers in Neuroendocrinology 2001, 22, 248.

(10) Xiao, D.; Qu, X.; Weber, H. C. Cellular Signalling 2003, 15, 945.

(11) Bhattacharyya, K.; Goldschmidt, B. S.; Hannink, M.; Alexander, S.; Viator, J. A. Clinics in Laboratory Medicine 2012, 32, 89.

(12) Hong, B.; Zu, Y. Theranostics 2013, 3, 377.

(13) Wu, X.; Luo, L.; Yang, S.; Ma, X.; Li, Y.; Dong, C.; Tian, Y.; Zhang, L. e.; Shen, Z.; Wu, A. ACS Applied Materials \& Interfaces 2015, 7, 9965.

(14) Huang, X.; El-Sayed, M. A. Journal of Advanced Research 2010, 1, 13.

(15) Harmon, B. V.; Takano, Y. S.; Winterford, C. M.; Gobe, G. C. International journal of radiation biology 1991, 59, 489.

(16) Sakaguchi, Y.; Stephens, L. C.; Makino, M.; Kaneko, T.; Strebel, F. R.; Danhauser, L. L.; Jenkins, G. N.; Bull, J. M. Cancer research 1995, 55, 5459.

(17) Quinto, C. A.; Mohindra, P.; Tong, S.; Bao, G. Nanoscale 2015, 7, 12728.

(18) Maeda, H.; Wu, J.; Sawa, T.; Matsumura, Y.; Hori, K. Journal of controlled release : official journal of the Controlled Release Society 2000, 65, 271.

(19) Tanaka, T.; Shiramoto, S.; Miyashita, M.; Fujishima, Y.; Kaneo, Y. International journal of pharmaceutics 2004, 277, 39.

(20) Nune, S. K.; Gunda, P.; Thallapally, P. K.; Lin, Y. Y.; Forrest, M. L.; Berkland, C. J. Expert opinion on drug delivery 2009, 6, 1175. 
(21) Homan, K.; Shah, J.; Gomez, S.; Gensler, H.; Karpiouk, A.; BrannonPeppas, L.; Emelianov, S. Journal of biomedical optics 2010, 15, 021316.

(22) Ling, Y.; Wei, K.; Luo, Y.; Gao, X.; Zhong, S. Biomaterials 2011, 32, 7139.

(23) Wang, A. Z.; Gu, F.; Zhang, L.; Chan, J. M.; Radovic-Moreno, A.; Shaikh, M. R.; Farokhzad, O. C. Expert opinion on biological therapy 2008, 8, 1063.

(24) Yu, M. K.; Park, J.; Jon, S. Theranostics 2012, 2, 3.

(25) Beer, A. J.; Grosu, A. L.; Carlsen, J.; Kolk, A.; Sarbia, M.; Stangier, I.; Watzlowik, P.; Wester, H. J.; Haubner, R.; Schwaiger, M. Clinical cancer research : an official journal of the American Association for Cancer Research 2007, 13, 6610.

(26) Kenny, L. M.; Coombes, R. C.; Oulie, I.; Contractor, K. B.; Miller, M.; Spinks, T. J.; McParland, B.; Cohen, P. S.; Hui, A. M.; Palmieri, C.; Osman, S.; Glaser, M.; Turton, D.; Al-Nahhas, A.; Aboagye, E. O. Journal of nuclear medicine : official publication, Society of Nuclear Medicine 2008, 49, 879.

(27) Yang, Y. S.; Zhang, X.; Xiong, Z.; Chen, X. Nucl Med Biol 2006, 33, 371.

(28) Zhang, X.; Cai, W.; Cao, F.; Schreibmann, E.; Wu, Y.; Wu, J. C.; Xing, L.; Chen, X. Journal of nuclear medicine : official publication, Society of Nuclear Medicine 2006, 47, 492.

(29) Veiseh, O.; Sun, C.; Fang, C.; Bhattarai, N.; Gunn, J.; Kievit, F.; Du, K.; Pullar, B.; Lee, D.; Ellenbogen, R. G.; Olson, J.; Zhang, M. Cancer research 2009, 69, 6200.

(30) Wang, A. Z.; Bagalkot, V.; Vasilliou, C. C.; Gu, F.; Alexis, F.; Zhang, L.; Shaikh, M.; Yuet, K.; Cima, M. J.; Langer, R.; Kantoff, P. W.; Bander, N. H.; Jon, S.; Farokhzad, O. C. ChemMedChem 2008, 3, 1311.

(31) Hwang, D. W.; Ko, H. Y.; Lee, J. H.; Kang, H.; Ryu, S. H.; Song, I. C.; Lee, D. S.; Kim, S. Journal of nuclear medicine : official publication, Society of Nuclear Medicine 2010, 51, 98.

(32) Raynal, I.; Prigent, P.; Peyramaure, S.; Najid, A.; Rebuzzi, C.; Corot, C. Investigative radiology 2004, 39, 56.

(33) Peng, X. H.; Qian, X.; Mao, H.; Wang, A. Y.; Chen, Z. G.; Nie, S.; Shin, D. M. Int J Nanomedicine 2008, 3, 311.

(34) Roser, M.; Fischer, D.; Kissel, T. European journal of pharmaceutics and biopharmaceutics : official journal of Arbeitsgemeinschaft fur Pharmazeutische Verfahrenstechnik e.V 1998, 46, 255.

(35) Shi, X.; Thomas, T. P.; Myc, L. A.; Kotlyar, A.; Baker, J. R., Jr. Physical chemistry chemical physics : PCCP 2007, 9, 5712.

(36) Xu, M.; Wang, L. V. Review of Scientific Instruments 2006, 77, 041101.

(37) Zanganeh, S.; Li, H.; Kumavor, P. D.; Alqasemi, U.; Aguirre, A.; Mohammad, I.; Stanford, C.; Smith, M. B.; Zhu, Q. Journal of biomedical optics 2013, 18, 096006.

(38) Jeon, M.; Song, W.; Huynh, E.; Kim, J.; Kim, J.; Helfield, B. L.; Leung, B. Y.; Goertz, D. E.; Zheng, G.; Oh, J.; Lovell, J. F.; Kim, C. Journal of biomedical optics 2014, 19, 16005.

(39) Wu, D.; Huang, L.; Jiang, M.; Jiang, H. International Journal of Molecular Sciences 2014, 15, 23616. 
(40) Link, S.; El-Sayed, M. A. The Journal of Physical Chemistry B 1999, 103, 8410.

(41) Ye, X.; Gao, Y.; Chen, J.; Reifsnyder, D. C.; Zheng, C.; Murray, C. B. Nano letters 2013, 13, 2163.

(42) Oldenburg, S. J.; Averitt, R. D.; Westcott, S. L.; Halas, N. J. Chemical Physics Letters 1998, 288, 243.

(43) Ah, C. S.; Yun, Y. J.; Park, H. J.; Kim, W.-J.; Ha, D. H.; Yun, W. S. Chemistry of Materials 2005, 17, 5558.

(44) Wang, Y.; Liu, Y.; Luehmann, H.; Xia, X.; Brown, P.; Jarreau, C.; Welch, M.; Xia, Y. ACS Nano 2012, 6, 5880.

(45) Nehl, C. L.; Liao, H.; Hafner, J. H. Nano letters 2006, 6, 683.

(46) Wang, S.; Huang, P.; Nie, L.; Xing, R.; Liu, D.; Wang, Z.; Lin, J.; Chen, S.; Niu, G.; Lu, G.; Chen, X. Advanced Materials 2013, 25, 3055.

(47) Zhang, Q.; Iwakuma, N.; Sharma, P.; Moudgil, B. M.; Wu, C.; McNeill, J.; Jiang, H.; Grobmyer, S. R. Nanotechnology 2009, 20, 395102.

(48) Kim, C.; Cho, E. C.; Chen, J.; Song, K. H.; Au, L.; Favazza, C.; Zhang, Q.; Cobley, C. M.; Gao, F.; Xia, Y.; Wang, L. V. ACS Nano 2010, 4, 4559.

(49) Zhang, Y. S.; Wang, Y.; Wang, L.; Wang, Y.; Cai, X.; Zhang, C.; Wang, L. V.; Xia, Y. Theranostics 2013, 3, 532.

(50) Thomas, T. S.; Dale, P. S.; Weight, R. M.; Atasoy, U.; Magee, J.; Viator, J. A. 2008; Vol. 6856, p 685609.

(51) Viator, J. A.; Gupta, S.; Goldschmidt, B. S.; Bhattacharyyal, K.; Kannan, R.; Shukla, R.; Dale, P. S.; Boote, E.; Katti, K. Journal of biomedical nanotechnology 2010, 6, 187.

(52) McCormack, D. R.; Bhattacharyya, K.; Kannan, R.; Katti, K.; Viator, J. A. Lasers in surgery and medicine 2011, 43, 333.

(53) Shashkov, E. V.; Everts, M.; Galanzha, E. I.; Zharov, V. P. Nano letters 2008, 8, 3953.

(54) Grootendorst, D. J.; Jose, J.; Fratila, R. M.; Visscher, M.; Velders, A. H.; Ten Haken, B.; Van Leeuwen, T. G.; Steenbergen, W.; Manohar, S.; Ruers, T. J. Contrast media \& molecular imaging 2013, 8, 83.

(55) Zhu, B.; Sevick-Muraca, E. M. The British journal of radiology 2015, 88, 20140547.

(56) Yi, X.; Wang, F.; Qin, W.; Yang, X.; Yuan, J. Int J Nanomedicine 2014, 9, 1347.

(57) Siegel, R. L.; Miller, K. D.; Jemal, A. CA: A Cancer Journal for Clinicians $2015,65,5$.

(58) New England Journal of Medicine 2012, 366, 2228.

(59) Liong, M.; Lu, J.; Kovochich, M.; Xia, T.; Ruehm, S. G.; Nel, A. E.; Tamanoi, F.; Zink, J. I. ACS Nano 2008, 2, 889.

(60) Doane, T. L.; Burda, C. Chemical Society reviews 2012, 41, 2885.

(61) Ma, X.; Zhao, Y.; Liang, X. J. Accounts of chemical research 2011, 44, 1114.

(62) Nakamura, H.; Ito, N.; Kotake, F.; Mizokami, Y.; Matsuoka, T. Journal of gastroenterology 2000, 35, 849. 
(63) Xie, J.; Chen, K.; Huang, J.; Lee, S.; Wang, J.; Gao, J.; Li, X.; Chen, X. Biomaterials 2010, 31, 3016.

(64) Weissleder, R.; Stark, D. D.; Engelstad, B. L.; Bacon, B. R.; Compton, C. C.; White, D. L.; Jacobs, P.; Lewis, J. AJR. American journal of roentgenology 1989, 152, 167.

(65) Tong, S.; Hou, S.; Zheng, Z.; Zhou, J.; Bao, G. Nano letters 2010, 10, 4607.

(66) Elzoghby, A. O.; El-Fotoh, W. S.; Elgindy, N. A. Journal of controlled release : official journal of the Controlled Release Society 2011, 153, 206.

(67) Huang, J.; Wang, L.; Lin, R.; Wang, A. Y.; Yang, L.; Kuang, M.; Qian, W.; Mao, H. ACS applied materials \& interfaces 2013, 5, 4632.

(68) Smith, C. J.; Sieckman, G. L.; Owen, N. K.; Hayes, D. L.; Mazuru, D. G.; Kannan, R.; Volkert, W. A.; Hoffman, T. J. Cancer research 2003, 63, 4082.

(69) Hoffman, T. J.; Gali, H.; Smith, C. J.; Sieckman, G. L.; Hayes, D. L.; Owen, N. K.; Volkert, W. A. Journal of nuclear medicine : official publication, Society of Nuclear Medicine 2003, 44, 823.

(70) Mansi, R.; Wang, X.; Forrer, F.; Waser, B.; Cescato, R.; Graham, K.; Borkowski, S.; Reubi, J. C.; Maecke, H. R. European journal of nuclear medicine and molecular imaging 2011, 38, 97.

(71) Chen, X.; Park, R.; Hou, Y.; Tohme, M.; Shahinian, A. H.; Bading, J. R.; Conti, P. S. Journal of nuclear medicine : official publication, Society of Nuclear Medicine 2004, 45, 1390.

(72) Prasanphanich, A. F.; Nanda, P. K.; Rold, T. L.; Ma, L.; Lewis, M. R.; Garrison, J. C.; Hoffman, T. J.; Sieckman, G. L.; Figueroa, S. D.; Smith, C. J. Proceedings of the National Academy of Sciences of the United States of America 2007, 104, 12462.

(73) Honer, M.; Mu, L.; Stellfeld, T.; Graham, K.; Martic, M.; Fischer, C. R.; Lehmann, L.; Schubiger, P. A.; Ametamey, S. M.; Dinkelborg, L.; Srinivasan, A.; Borkowski, S. Journal of nuclear medicine : official publication, Society of Nuclear Medicine 2011, 52, 270.

(74) Johnson, C. V.; Shelton, T.; Smith, C. J.; Ma, L.; Perry, M. C.; Volkert, W. A.; Hoffman, T. J. Cancer biotherapy \& radiopharmaceuticals 2006, 21, 155.

(75) Zhang, H.; Chen, J.; Waldherr, C.; Hinni, K.; Waser, B.; Reubi, J. C.; Maecke, H. R. Cancer research 2004, 64, 6707.

(76) Durkan, K.; Jiang, Z.; Rold, T. L.; Sieckman, G. L.; Hoffman, T. J.; Bandari, R. P.; Szczodroski, A. F.; Liu, L.; Miao, Y.; Reynolds, T. S.; Smith, C. J. Nucl Med Biol 2014, 41, 133.

(77) Nanda, P. K.; Pandey, U.; Bottenus, B. N.; Rold, T. L.; Sieckman, G. L.; Szczodroski, A. F.; Hoffman, T. J.; Smith, C. J. Nuclear Medicine and Biology 2012, 39, 461.

(78) Jackson, A. B.; Nanda, P. K.; Rold, T. L.; Sieckman, G. L.; Szczodroski, A. F.; Hoffman, T. J.; Chen, X.; Smith, C. J. Nucl Med Biol 2012, 39, 377.

(79) Nanda, P. K.; Wienhoff, B. E.; Rold, T. L.; Sieckman, G. L.; Szczodroski, A. F.; Hoffman, T. J.; Rogers, B. E.; Smith, C. J. In vivo (Athens, Greece) 2012, 26, 583.

(80) Bandari, R. P.; Jiang, Z.; Reynolds, T. S.; Bernskoetter, N. E.; Szczodroski, A. F.; Bassuner, K. J.; Kirkpatrick, D. L.; Rold, T. L.; Sieckman, G. L.; Hoffman, T. J.; Connors, J. P.; Smith, C. J. Nucl Med Biol 2014, 41, 355. 
(81) Cai, Q. Y.; Yu, P.; Besch-Williford, C.; Smith, C. J.; Sieckman, G. L.; Hoffman, T. J.; Ma, L. The Prostate 2013, 73, 842.

(82) Keereweer, S.; Hutteman, M.; Kerrebijn, J. D.; van de Velde, C. J.; Vahrmeijer, A. L.; Lowik, C. W. Current pharmaceutical biotechnology 2012, 13, 498.

(83) Mieog, J. S. D.; Troyan, S. L.; Hutteman, M.; Donohoe, K. J.; van der Vorst, J. R.; Stockdale, A.; Liefers, G.-J.; Choi, H. S.; Gibbs-Strauss, S. L.; Putter, H.; Gioux, S.; Kuppen, P. J. K.; Ashitate, Y.; Löwik, C. W. G. M.; Smit, V. T. H. B. M.; Oketokoun, R.; Ngo, L. H.; van de Velde, C. J. H.; Frangioni, J. V.; Vahrmeijer, A. L. Annals of Surgical Oncology 2011, 18, 2483.

(84) Seibel, E. J.; Brentnall, T. A.; Dominitz, J. A. Gastrointestinal endoscopy clinics of North America 2009, 19, 299.

(85) Mahmood, U. Gastroenterology 2010, 138, 419.

(86) Weissleder, R.; Ntziachristos, V. Nature medicine 2003, 9, 123.

(87) Jiao, Y.; Sun, Y.; Tang, X.; Ren, Q.; Yang, W. Small 2015, 11, 1962.

(88) Weissleder, R.; Elizondo, G.; Wittenberg, J.; Rabito, C. A.; Bengele, H. H.; Josephson, L. Radiology 1990, 175, 489.

(89) Thomas, R.; Park, I. K.; Jeong, Y. Y. Int J Mol Sci 2013, 14, 15910.

(90) !!! INVALID CITATION !!! \{\}.

(91) Park, Y. I.; Piao, Y.; Lee, N.; Yoo, B.; Kim, B. H.; Choi, S. H.; Hyeon, T. Journal of Materials Chemistry 2011, 21, 11472.

3, 595.

(92) Li, L.; Jiang, W.; Luo, K.; Song, H.; Lan, F.; Wu, Y.; Gu, Z. Theranostics 2013,

(93) Erspamer, V.; Melchiorri, P.; Sopranzi, N. British journal of pharmacology $1973,48,438$.

(94) Yang, C. Y.; Hsiao, J. K.; Tai, M. F.; Chen, S. T.; Cheng, H. Y.; Wang, J. L.; Liu, H. M. Mol Imaging Biol 2011, 13, 443.

(95) Huang, G.; Chen, H.; Dong, Y.; Luo, X.; Yu, H.; Moore, Z.; Bey, E. A.; Boothman, D. A.; Gao, J. Theranostics 2013, 3, 116.

(96) Liu, G.; Gao, J.; Ai, H.; Chen, X. Small 2013, 9, 1533.

(97) Siegel, R. L.; Miller, K. D.; Jemal, A. CA Cancer J Clin 2016, 66, 7.

(98) Welch, H. G.; Albertsen, P. C. Journal of the National Cancer Institute 2009, 101, 1325.

(99) Kozlowski, P.; Chang, S. D.; Jones, E. C.; Berean, K. W.; Chen, H.; Goldenberg, S. L. Journal of magnetic resonance imaging : JMRI 2006, 24, 108.

(100) Anastasi, A.; Erspamer, V.; Bucci, M. Archives of biochemistry and biophysics 1972, 148, 443.

(101) Gonzalez, N.; Moody, T. W.; Igarashi, H.; Ito, T.; Jensen, R. T. Current opinion in endocrinology, diabetes, and obesity 2008, 15, 58.

(102) Ohki-Hamazaki, H.; Iwabuchi, M.; Maekawa, F. The International journal of developmental biology 2005, 49, 293.

(103) Smith, C. J.; Volkert, W. A.; Hoffman, T. J. Nucl Med Biol 2003, 30, 861.

(104) Von Schrenck, T.; Heinz-Erian, P.; Moran, T.; Mantey, S. A.; Gardner, J. D.; Jensen, R. T. The American journal of physiology 1989, 256, G747. 
(105) Spindel, E. R.; Giladi, E.; Brehm, P.; Goodman, R. H.; Segerson, T. P. Molecular endocrinology (Baltimore, Md.) 1990, 4, 1956.

(106) Sun, B.; Halmos, G.; Schally, A. V.; Wang, X.; Martinez, M. The Prostate 2000, 42, 295.

(107) Moody, T. W.; Bertness, V.; Carney, D. N. Peptides 1983, 4, 683.

(108) Sancho, V.; Di Florio, A.; Moody, T. W.; Jensen, R. T. Current drug delivery 2011, 8, 79.

(109) Reubi, J. C. Endocrine reviews 2003, 24, 389.

(110) Hohla, F.; Schally, A. V. Cell cycle (Georgetown, Tex.) 2010, 9, 1738.

(111) Ma, L.; Yu, P.; Veerendra, B.; Rold, T. L.; Retzloff, L.; Prasanphanich, A.; Sieckman, G.; Hoffman, T. J.; Volkert, W. A.; Smith, C. J. Molecular imaging 2007, 6, 171.

(112) Winkelmann, C. T.; Figueroa, S. D.; Sieckman, G. L.; Rold, T. L.; Hoffman, T. J. Mol Imaging Biol 2012, 14, 667.

(113) Lane, S. R.; Nanda, P.; Rold, T. L.; Sieckman, G. L.; Figueroa, S. D.; Hoffman, T. J.; Jurisson, S. S.; Smith, C. J. Nucl Med Biol 2010, 37, 751.

(114) Kujala, N.; Zhai, H.; Smith, C.; Prasanphanich, A.; Sieckman, G.; Hoffman, T.; Volkert, W.; Ma, L.; Yu, P. 2009; Vol. 7190, p 719010.

(115) Prasanphanich, A. F.; Retzloff, L.; Lane, S. R.; Nanda, P. K.; Sieckman, G. L.; Rold, T. L.; Ma, L.; Figueroa, S. D.; Sublett, S. V.; Hoffman, T. J.; Smith, C. J. Nuclear medicine and biology 2009, 36, 171.

(116) Abd-Elgaliel, W. R.; Gallazzi, F.; Garrison, J. C.; Rold, T. L.; Sieckman, G. L.; Figueroa, S. D.; Hoffman, T. J.; Lever, S. Z. Bioconjug Chem 2008, 19, 2040.

(117) Garrison, J. C.; Rold, T. L.; Sieckman, G. L.; Naz, F.; Sublett, S. V.; Figueroa, S. D.; Volkert, W. A.; Hoffman, T. J. Bioconjug Chem 2008, 19, 1803.

(118) Lane, S. R.; Veerendra, B.; Rold, T. L.; Sieckman, G. L.; Hoffman, T. J.; Jurisson, S. S.; Smith, C. J. Nuclear medicine and biology 2008, 35, 263.

(119) Garrison, J. C.; Rold, T. L.; Sieckman, G. L.; Figueroa, S. D.; Volkert, W. A.; Jurisson, S. S.; Hoffman, T. J. Journal of nuclear medicine : official publication, Society of Nuclear Medicine 2007, 48, 1327.

(120) Smith, C. J.; Gali, H.; Sieckman, G. L.; Hayes, D. L.; Owen, N. K.; Mazuru, D. G.; Volkert, W. A.; Hoffman, T. J. Nucl Med Biol 2003, 30, 101.

(121) Smith, C. J.; Sieckman, G. L.; Owen, N. K.; Hayes, D. L.; Mazuru, D. G.; Volkert, W. A.; Hoffman, T. J. Anticancer research 2003, 23, 63.

(122) Varasteh, Z.; Åberg, O.; Velikyan, I.; Lindeberg, G.; Sörensen, J.; Larhed, M.; Antoni, G.; Sandström, M.; Tolmachev, V.; Orlova, A. PLoS ONE 2013, 8, e81932.

(123) Abiraj, K.; Mansi, R.; Tamma, M. L.; Fani, M.; Forrer, F.; Nicolas, G.; Cescato, R.; Reubi, J. C.; Maecke, H. R. Journal of nuclear medicine : official publication, Society of Nuclear Medicine 2011, 52, 1970.

(124) Marsouvanidis, P. J.; Nock, B. A.; Hajjaj, B.; Fehrentz, J.-A.; Brunel, L.; M'Kadmi, C.; van der Graaf, L.; Krenning, E. P.; Maina, T.; Martinez, J.; de Jong, M. Journal of medicinal chemistry 2013, 56, 2374.

(125) Varasteh, Z.; Velikyan, I.; Lindeberg, G.; Sörensen, J.; Larhed, M.; Sandström, M.; Selvaraju, R. K.; Malmberg, J.; Tolmachev, V.; Orlova, A. Bioconjugate Chemistry 2013, 24, 1144. 
(126) Shrivastava, A.; Ding, H.; Kothandaraman, S.; Wang, S. H.; Gong, L.; Williams, M.; Milum, K.; Zhang, S.; Tweedle, M. F. Mol Imaging Biol 2014, 16, 661.

(127) Prasanphanich, A. F.; Lane, S. R.; Figueroa, S. D.; Ma, L.; Rold, T. L.; Sieckman, G. L.; Hoffman, T. J.; McCrate, J. M.; Smith, C. J. In vivo (Athens, Greece) 2007, $21,1$.

(128) Achilefu, S.; Jimenez, H. N.; Dorshow, R. B.; Bugaj, J. E.; Webb, E. G.; Wilhelm, R. R.; Rajagopalan, R.; Johler, J.; Erion, J. L. Journal of medicinal chemistry 2002, 45, 2003.

(129) Mehlen, P.; Puisieux, A. Nat Rev Cancer 2006, 6, 449.

(130) Pantel, K.; Speicher, M. R. Oncogene 2016, 35, 1216.

(131) Fidler, I. J. Nat Rev Cancer 2003, 3, 453.

(132) Paterlini-Brechot, P.; Benali, N. L. Cancer letters 2007, 253, 180.

(133) Galanzha, E. I.; Zharov, V. P. Cancers 2013, 5, 1691.

(134) Yu, M.; Stott, S.; Toner, M.; Maheswaran, S.; Haber, D. A. The Journal of cell biology 2011, 192, 373.

(135) Ferro, P.; Franceschini, M. C.; Bacigalupo, B.; Dessanti, P.; Falco, E.; Fontana, V.; Gianquinto, D.; Pistillo, M. P.; Fedeli, F.; Roncella, S. Anticancer research 2010, 30, 2377.

(136) Okegawa, T.; Hayashi, K.; Hara, H.; Nutahara, K.; Higashihara, E. International journal of urology : official journal of the Japanese Urological Association 2010, 17, 254.

(137) Oh, J.-T.; Li, M.-L.; Zhang, H. F.; Maslov, K.; Stoica, G.; Wang, L. V. Journal of biomedical optics 2006, 11, 034032.

(138) Grootendorst, D. J.; Jose, J.; Wouters, M. W.; van Boven, H.; Van der Hage, J.; Van Leeuwen, T. G.; Steenbergen, W.; Manohar, S.; Ruers, T. J. Lasers in surgery and medicine 2012, 44, 541.

(139) Ku, G.; Wang, X.; Xie, X.; Stoica, G.; Wang, L. V. Applied optics 2005, 44, 770.

(140) Lao, Y.; Xing, D.; Yang, S.; Xiang, L. Physics in medicine and biology 2008, $53,4203$.

(141) Li, W.; Chen, X. Nanomedicine (London, England) 2015, 10, 299.

(142) Alkilany, A. M.; Murphy, C. J. Journal of Nanoparticle Research 2010, 12, 2313.

(143) Nedosekin, D. A.; Sarimollaoglu, M.; Ye, J. H.; Galanzha, E. I.; Zharov, V. P. Cytometry. Part A : the journal of the International Society for Analytical Cytology 2011, $79,825$.

(144) Zharov, V. P.; Galanzha, E. I.; Shashkov, E. V.; Khlebtsov, N. G.; Tuchin, V. V. Optics letters 2006, 31, 3623.

(145) Galanzha, E. I.; Shashkov, E. V.; Spring, P. M.; Suen, J. Y.; Zharov, V. P. Cancer research 2009, 69, 7926.

(146) Mahmoudi, M.; Simchi, A.; Milani, A. S.; Stroeve, P. Journal of Colloid and Interface Science 2009, 336, 510.

(147) Mahmoudi, M.; Hofmann, H.; Rothen-Rutishauser, B.; Petri-Fink, A. Chem Rev 2012, 112, 2323. 
(148) Arbab, A. S.; Wilson, L. B.; Ashari, P.; Jordan, E. K.; Lewis, B. K.; Frank, J. A. NMR Biomed 2005, 18, 383.

(149) Laurent, S.; Forge, D.; Port, M.; Roch, A.; Robic, C.; Vander Elst, L.; Muller, R. N. Chemical Reviews 2008, 108, 2064.

(150) Laurent, S.; Dutz, S.; Hafeli, U. O.; Mahmoudi, M. Advances in colloid and interface science 2011, 166, 8.

(151) McBain, S. C.; Yiu, H. H. P.; Dobson, J. International Journal of Nanomedicine 2008, 3, 169.

(152) Alwi, R.; Telenkov, S.; Mandelis, A.; Leshuk, T.; Gu, F.; Oladepo, S.; Michaelian, K. Biomedical Optics Express 2012, 3, 2500.

(153) Galanzha, E. I.; Shashkov, E. V.; Kelly, T.; Kim, J.-W.; Yang, L.; Zharov, V. P. Nat Nano 2009, 4, 855.

(154) Chen, Y.-S.; Frey, W.; Kim, S.; Kruizinga, P.; Homan, K.; Emelianov, S. Nano letters 2011, 11, 348.

(155) Wang, X.; Qian, X.; Beitler, J. J.; Chen, Z. G.; Khuri, F. R.; Lewis, M. M.; Shin, H. J.; Nie, S.; Shin, D. M. Cancer research 2011, 71, 1526.

(156) http://www.cancer.gov/about-cancer/diagnosis-staging/staging

(157) Lin, K.; Luu, A.; Baidoo, K.; Gargari, H.; Chen, M.; Brenneman, K.; Pili, R.; Pomper, M.; Carducci, M.; Wagner, H. Bioconjugate Chem 2005, 16, 43.

(158) Scott, N.; Millward, E.; Cartwright, E.; Preston, S.; Coletta, P. Journal of Clinical Pathology 2004, 57, 189. 


\section{Vita}

Hang Xu was born in June 1991, in Jiangsu, China. He graduated from Zhenjiang High School in 2009 and received his bachelor degree in 2013 from the Department of Materials Science and Technology in East China University of Science and Technology, which is a top university in the field of chemical engineering and chemistry-related technologies in China. He was a visiting student in the Department of Chemical Engineering in the University of Missouri in Columbia from August 2012 to July 2013. He was subsequently admitted to graduate school there in August 2013. In an opportunity working at Dr. Lixin Ma's lab, Hang's interest in developing novel molecular imaging probes for early detection of cancer was stimulated. He then was enrolled in the Ph.D. program in the Department of Chemical Engineering in University of Missouri, Columbia in January 2014.

Hang won a Center for Molecular Imaging Innovation and Translation (CMIIT) Young Investigator Award $2^{\text {nd }}$ place in 2015 at the Society of Nuclear Medicine and Molecular Imaging (SNMMI) conference. He also won a second place Poster Award of Missouri Life Science Week in 2015. He has published three conference abstracts, and this Ph.D. thesis will result in three manuscripts for research journal publications.

Hang is aspired to pursue a career in imaging agent research and development in industry and academia, and his desire about reducing cancer death by improving early detection of cancer will continue to inspire him in the future of his career. 